\title{
Finite energy foliations on overtwisted contact manifolds
}

\author{
CHRIS WENDL
}

\begin{abstract}
We develop a method for preserving pseudoholomorphic curves in contact 3-manifolds under surgery along transverse links. This makes use of a geometrically natural boundary value problem for holomorphic curves in a 3-manifold with stable Hamiltonian structure, where the boundary conditions are defined by 1-parameter families of totally real surfaces. The technique is applied here to construct a finite energy foliation for every closed overtwisted contact 3-manifold.
\end{abstract}

32Q65; 57R17

\section{Introduction}

\subsection{Definitions and main result}

Finite energy foliations of contact 3-manifolds were introduced by Hofer, Wysocki and Zehnder in [16], where they were shown to exist for generic tight three-spheres, giving powerful consequences for the Reeb dynamics. The present work is a step toward generalizing such existence results: we prove that for every closed overtwisted contact 3-manifold, one can choose a contact form (of Morse-Bott type) and compatible complex multiplication such that a stable finite energy foliation exists.

Fix a closed oriented 3-manifold $M$ with a cooriented, positive contact structure $\xi$ : this is by definition the kernel of a smooth 1 -form $\lambda$ which satisfies $\lambda \wedge d \lambda>0$. The choice of contact form defines the Reeb vector field $X$ by the conditions

$$
d \lambda(X, \cdot) \equiv 0 \quad \text { and } \quad \lambda(X) \equiv 1 .
$$

Then the flow of $X$ preserves $\xi$, along with the symplectic vector bundle structure on $\xi \rightarrow M$ defined by $d \lambda$.

Recall that a contact structure $\xi$ is called overtwisted if there exists an overtwisted disk: an embedded disk $\mathcal{D} \subset M$ such that for all $m \in \partial \mathcal{D}, T_{m}(\partial \mathcal{D}) \subset \xi_{m}$ but $T_{m} \mathcal{D} \neq \xi_{m}$. By Eliashberg's classification result [4], contactomorphism classes of overtwisted contact structures on $M$ are in one-to-one correspondence with homotopy classes of cooriented 2-plane distributions.

The following is the main result of this paper. 
Theorem 1.1 Suppose $(M, \xi)$ is a closed oriented 3-manifold with a positive overtwisted contact structure. Then there exists a contact form $\lambda$ and admissible complex multiplication $J$ such that $(M, \lambda, J)$ admits a stable finite energy foliation of MorseBott type. The foliation has precisely one nondegenerate asymptotic orbit and one or more Morse-Bott tori of asymptotic orbits, and every leaf is either an orbit cylinder or an index 2 finite energy sphere with distinct simply covered asymptotic orbits, all positive.

We will spend the rest of Section 1.1 explaining the definitions needed to understand this statement.

Denote the time- $t$ flow of $X$ by $\varphi_{X}^{t}$, and recall that a closed orbit $x: \mathbb{R} \rightarrow M$ of $X$ with period $T>0$ is called nondegenerate if the linearized return map $\left.d \varphi_{X}^{T}(x(0))\right|_{\xi_{x(0)}}$ does not have 1 in its spectrum. More generally, a Morse-Bott manifold of T-periodic orbits is a submanifold $N \subset M$ tangent to $X$ such that $\left.\varphi_{X}^{T}\right|_{N}$ is the identity, and for every $m \in N$,

$$
T_{m} N=\operatorname{ker}\left(d \varphi_{X}^{T}(m)-\mathbb{1}\right) .
$$

In this paper we shall deal exclusively with situations where $N$ is a circle (ie a nondegenerate orbit) or a two-dimensional torus. For the latter case, one can show (see Wendl [28]) that all orbits in $N$ have the same minimal period $\tau>0$, and $N$ is a Morse-Bott family of $k \tau$-periodic orbits for all $k \in \mathbb{N}$. Thus we will call such submanifolds Morse-Bott tori without reference to the period, and a single closed orbit will be called simply Morse-Bott if it either is nondegenerate or belongs to a Morse-Bott torus.

The symplectization of $M$ is the open 4-manifold $\mathbb{R} \times M$ with symplectic structure $d\left(e^{a} \lambda\right)$, where $a$ denotes the coordinate on the $\mathbb{R}$-component. We consider a natural class of $\mathbb{R}$-invariant almost complex structures compatible with this symplectic form, defined as follows. Note the choice of contact form $\lambda$ defines a splitting

$$
T(\mathbb{R} \times M)=(\mathbb{R} \oplus \mathbb{R} X) \oplus \xi,
$$

where the first factor also comes with a natural trivialization. An admissible complex multiplication is a choice of complex structure $J$ for the bundle $\xi \rightarrow M$, compatible with the symplectic structure, ie we require that $d \lambda(\cdot, J \cdot)$ define a bundle metric. Given any such choice, we define an almost complex structure $\widetilde{J}$ on $\mathbb{R} \times M$ in terms of the above splitting and trivialization by

$$
\widetilde{J}=i \oplus J,
$$

where $i$ is understood as the natural complex structure acting on $\mathbb{C}=\mathbb{R}^{2}$. We will call $\widetilde{J}$ the almost complex structure associated to $\lambda$ and $J$. 
Given such a structure, we consider $\widetilde{J}$-holomorphic curves

$$
\tilde{u}=(a, u):(\dot{\Sigma}, j) \rightarrow(\mathbb{R} \times M, \widetilde{J}),
$$

where the domain $\dot{\Sigma}=\Sigma \backslash \Gamma$ is a Riemann surface $(\Sigma, j)$ with a discrete set of points $\Gamma \subset \Sigma$ removed. The energy of such a curve is defined as

$$
E(\tilde{u})=\sup _{\varphi \in \mathcal{T}} \int_{\dot{\Sigma}} \tilde{u}^{*} d(\varphi \lambda),
$$

where $\mathcal{T}:=\left\{\varphi \in C^{\infty}(\mathbb{R},[0,1]) \mid \varphi^{\prime} \geq 0\right\}$. An easy computation shows that the integrand is nonnegative whenever $\tilde{u}$ is $\widetilde{J}$-holomorphic, and such a curve is constant if and only if $E(\widetilde{u})=0$. When $\Sigma$ is closed, $\widetilde{J}$-holomorphic curves $\tilde{u}: \dot{\Sigma} \rightarrow \mathbb{R} \times M$ with $E(\widetilde{u})<\infty$ are called finite energy surfaces. By results in Hofer [10] and Hofer-Wysocki-Zehnder $[11 ; 14]$, these have nicely controlled asymptotic behavior near the punctures, which can be described as follows. Denote by $\mathbb{D} \subset \mathbb{C}$ the closed unit disk with its natural complex structure, and let $\mathbb{D}_{r} \subset \mathbb{C}$ be the closed disk of radius $r$ for any $r>0$.

Proposition 1.2 Suppose $\tilde{u}=(a, u): \dot{\mathbb{D}}=\mathbb{D} \backslash\{0\} \rightarrow \mathbb{R} \times M$ is a $\widetilde{J}$-holomorphic map with $0<E(\tilde{u})<\infty$. If $\tilde{u}$ is bounded, then $\tilde{u}$ extends to a $\widetilde{J}$-holomorphic map $\mathbb{D} \rightarrow \mathbb{R} \times M$. Otherwise, $\tilde{u}$ is a proper map, and for every sequence $s_{k} \rightarrow \infty$ there is a subsequence such that the loops $t \mapsto u\left(e^{-2 \pi\left(s_{k}+i t\right)}\right)$ converge in $C^{\infty}\left(S^{1}, M\right)$ to a loop $t \mapsto x(Q t)$. Here $x: \mathbb{R} \rightarrow M$ is a periodic orbit of $X$ with period $T=|Q|$, where

$$
Q=-\lim _{\epsilon \rightarrow 0} \int_{\partial \mathbb{D}_{\epsilon}} u^{*} \lambda \neq 0 .
$$

Moreover, $t \mapsto a\left(e^{-2 \pi\left(s_{k}+i t\right)}\right) / s_{k}$ converges in $C^{\infty}\left(S^{1}, \mathbb{R}\right)$ to the constant map $t \mapsto$ $Q$.

If the orbit $x$ is Morse-Bott, then in fact the maps $t \mapsto u\left(e^{-2 \pi(s+i t)}\right)$ and $t \mapsto$ $a\left(e^{-2 \pi(s+i t)}\right) / s$ converge in $C^{\infty}\left(S^{1}\right)$ as $s \rightarrow \infty$.

The number $Q \in \mathbb{R} \backslash\{0\}$ appearing above is called the charge of the puncture, and we call the puncture positive/negative in accordance with the sign of $Q$. This defines a partition of the set of punctures:

$$
\Gamma=\Gamma^{+} \cup \Gamma^{-}
$$

and one can use the maximum principle to show that finite energy surfaces always have $\# \Gamma^{+} \geq 1$, cf Hofer-Wysocki-Zehnder [12]. 
The simplest example of a finite energy surface is the so-called orbit cylinder or trivial cylinder over a $T$-periodic orbit $x: \mathbb{R} \rightarrow M$. Indeed, it's easy to check that the map

$$
\tilde{u}: \mathbb{R} \times S^{1} \rightarrow \mathbb{R} \times M:(s, t) \mapsto(T s, x(T t))
$$

is $\widetilde{J}$-holomorphic and has finite energy; after reparametrization, it is a sphere with one positive puncture and one negative. Proposition 1.2 above is a precise way of saying that any finite energy surface looks approximately like an orbit cylinder near each puncture.

Definition 1.3 A finite energy foliation for $(M, \lambda, J)$ is a smooth two-dimensional foliation $\mathcal{F}$ of $\mathbb{R} \times M$ such that the following are true.

(1) Each leaf $F \in \mathcal{F}$ can be presented as the image of an embedded $\widetilde{J}$-holomorphic finite energy surface, and there exists a constant that bounds the energy of every leaf uniformly.

(2) For every leaf $F \in \mathcal{F}$, the set $\sigma+F:=\{(\sigma+a, m) \mid(a, m) \in F\}$ for $\sigma \in \mathbb{R}$ is also a leaf of the foliation, and thus either disjoint from or identical to $F$.

We shall often abuse notation and write $\tilde{u} \in \mathcal{F}$, meaning that the finite energy surface $\tilde{u}$ parametrizes a leaf of $\mathcal{F}$. The $\mathbb{R}$-invariance assumption says that $\tilde{u}=(a, u) \in \mathcal{F}$ if and only if $\widetilde{u}^{\sigma}:=(a+\sigma, u) \in \mathcal{F}$ for all $\sigma \in \mathbb{R}$. This has several consequences for the projection of $\mathcal{F}$ to the underlying contact manifold.

Proposition 1.4 Let $\mathcal{F}$ be a finite energy foliation.

(i) If $P \subset M$ is a periodic orbit which is an asymptotic limit for some leaf $\tilde{u} \in \mathcal{F}$, then the orbit cylinder $\mathbb{R} \times P$ is also a leaf of $\mathcal{F}$.

(ii) For each leaf $\tilde{u}=(a, u): \dot{\Sigma} \rightarrow \mathbb{R} \times M$ of $\mathcal{F}$ that is not an orbit cylinder, the map $u: \dot{\Sigma} \rightarrow M$ is embedded and does not intersect its asymptotic limits.

(iii) If $\tilde{u}=(a, u): \dot{\Sigma} \rightarrow \mathbb{R} \times M$ and $\tilde{v}=(b, v): \dot{\Sigma}^{\prime} \rightarrow \mathbb{R} \times M$ are two leaves of $\mathcal{F}$, then $u(\dot{\Sigma})$ and $v\left(\dot{\Sigma}^{\prime}\right)$ are either disjoint or identical.

The proofs of these properties are mostly straightforward exercises using positivity of intersections; we refer to Wendl [29] for details. The only detail not covered there is the fact that the maps $u$ : $\dot{\Sigma} \rightarrow M$ are not just injective but also embedded: for this one uses intersection theory to show that a critical point of $u$ at $z \in \dot{\Sigma}$ implies intersections between $(a, u)$ and $(a+\epsilon, u)$ near $z$ for small $\epsilon$; cf Wendl [28]. Denote by $\mathcal{P}_{\mathcal{F}} \subset M$ the union of all the closed orbits that occur as asymptotic limits for leaves of $\mathcal{F}$; equivalently, this is the projection down to $M$ of all the orbit cylinders in $\mathcal{F}$. Then Proposition 1.4 can be rephrased as follows. 
Corollary 1.5 If $\mathcal{F}$ is a finite energy foliation, then the projections of its leaves from $\mathbb{R} \times M$ to $M$ form a smooth foliation of $M \backslash \mathcal{P}_{\mathcal{F}}$.

To explain the stability condition, we need to introduce some more technical details. In the following, write periodic orbits of $X$ in the notation $P:=(x(\mathbb{R}), T)$ where $x: \mathbb{R} \rightarrow M$ is a $T$-periodic solution of the Reeb flow equation; note that $T$ need not be the minimal period, and the parameterization $x$ can always be changed by a time shift. We shall sometimes abuse notation and regard $P$ as a subset of $M$, keeping in mind that $T$ is also part of the data. Recall that if $P$ is nondegenerate, we can choose a unitary trivialization $\Phi$ of $\xi$ along $P$ and define the Conley-Zehnder index $\mu_{\mathrm{CZ}}^{\Phi}(P)$ as in Hofer-Wysocki-Zehnder [12]. Then a finite energy surface $\tilde{u}$ : $\dot{\Sigma} \rightarrow \mathbb{R} \times M$ with only nondegenerate asymptotic orbits $\left\{P_{z}\right\}_{z \in \Gamma}$ is assigned the Conley-Zehnder index

$$
\mu_{\mathrm{CZ}}(\widetilde{u})=\sum_{z \in \Gamma^{+}} \mu_{\mathrm{CZ}}^{\Phi}\left(P_{z}\right)-\sum_{z \in \Gamma^{-}} \mu_{\mathrm{CZ}}^{\Phi}\left(P_{z}\right),
$$

where the trivializations $\Phi$ are chosen so as to admit an extension to a global complex trivialization of $\xi$ along $\tilde{u}$; then $\mu_{\mathrm{CZ}}(\widetilde{u})$ doesn't depend on this choice.

This index can be extended to the Morse-Bott case in the following straightforward manner. Given $P$, define an admissible parameterization of $P$ to be any map $\mathbf{x}: S^{1} \rightarrow$ $P \subset M$ such that $\lambda(\dot{\mathbf{x}}) \equiv T$. This defines the so-called asymptotic operator

$$
\mathbf{A}_{\mathbf{x}}: \Gamma\left(\mathbf{x}^{*} \xi\right) \rightarrow \Gamma\left(\mathbf{x}^{*} \xi\right): v \mapsto-J\left(\nabla_{t} v-T \nabla_{v} X\right)
$$

where $\nabla$ is any symmetric connection on $M$; one can check that this expression gives a well defined section of $\mathbf{x}^{*} \xi$, not depending on $\nabla$. As an unbounded operator on $L^{2}\left(\mathbf{x}^{*} \xi\right)$ with domain $H^{1}\left(\mathbf{x}^{*} \xi\right), \mathbf{A}_{\mathbf{x}}$ is self-adjoint, with spectrum consisting of discrete real eigenvalues of finite multiplicity, accumulating only at infinity. The equation $\mathbf{A}_{\mathbf{x}} v=0$ then defines the linearized Reeb flow restricted to $\xi$ along $P$, and $P$ is nondegenerate if and only if $\operatorname{ker} \mathbf{A}_{\mathbf{x}}=\{0\}$. When this is the case, one can define the linearized Reeb flow purely in terms of the equation $\mathbf{A}_{\mathbf{x}} v=0$ and sensibly denote the Conley-Zehnder index by $\mu_{\mathrm{CZ}}^{\Phi}(P)=\mu_{\mathrm{CZ}}^{\Phi}\left(\mathbf{A}_{\mathbf{x}}\right)$. The key observation now is that for any $c \in \mathbb{R}$, the equation $\left(\mathbf{A}_{\mathbf{x}}-c\right) v=0$ also defines a linear Hamiltonian flow, and thus yields a well defined Conley-Zehnder index if $c$ is not an eigenvalue of $\mathbf{A}_{\mathbf{x}}$. Then if $P$ belongs to a Morse-Bott manifold $N \subset M$, we can pick any sufficiently small number $\epsilon>0$ and define the perturbed Conley-Zehnder indices

$$
\mu_{\mathrm{CZ}}^{\Phi \pm}(P)=\mu_{\mathrm{CZ}}^{\Phi}\left(\mathbf{A}_{\mathbf{x}} \pm \epsilon\right) .
$$

This doesn't depend on $\epsilon$ if the latter is sufficiently small, but does depend on the sign choice whenever $\operatorname{ker} \mathbf{A}_{\mathbf{x}}$ is nontrivial, ie when $P$ is degenerate. For $\tilde{u}$ with Morse-Bott 
asymptotic orbits, we now define its Conley-Zehnder index by

$$
\mu_{\mathrm{CZ}}(\widetilde{u})=\sum_{z \in \Gamma^{+}} \mu_{\mathrm{CZ}}^{\Phi-}\left(P_{z}\right)-\sum_{z \in \Gamma^{-}} \mu_{\mathrm{CZ}}^{\Phi+}\left(P_{z}\right)
$$

which is equal to the previous definition if all $P_{z}$ are nondegenerate.

The moduli space of finite energy surfaces $\mathcal{M}_{\widetilde{J}}$ is the set of equivalence classes $C=[(\Sigma, j, \Gamma, \tilde{u})]$, where $\tilde{u}:(\Sigma \backslash \Gamma, j) \rightarrow(\mathbb{R} \times M, \widetilde{J})$ is a finite energy surface, $\Gamma$ is assigned an ordering, and $(\Sigma, j, \Gamma, \widetilde{u}) \sim\left(\Sigma^{\prime}, j^{\prime}, \Gamma^{\prime}, \widetilde{u}^{\prime}\right)$ if and only if there is a biholomorphic map $\varphi:(\Sigma, j) \rightarrow\left(\Sigma^{\prime}, j^{\prime}\right)$ that takes $\Gamma$ to $\Gamma^{\prime}$ with ordering preserved and satisfies $\tilde{u}=\tilde{u}^{\prime} \circ \varphi$. We shall sometimes abuse notation and write $\tilde{u} \in \mathcal{M}_{\widetilde{J}}$ when there is no confusion.

To define a topology on $\mathcal{M}_{\widetilde{J}}$, first note that the punctured Riemann surface $(\dot{\Sigma}, j)$ can be regarded as a surface with cylindrical ends, which then admits a natural compactification. Indeed, for each $z \in \Gamma^{ \pm}$, pick a closed disk-like neighborhood $\mathcal{D}_{z}$ of $z$ in $\Sigma$ and a biholomorphic map $\dot{\mathcal{D}}_{z}:=\mathcal{D} \backslash\{z\} \rightarrow Z_{ \pm}$, where

$$
Z_{+}=[0, \infty) \times S^{1}, \quad Z_{-}=(-\infty, 0] \times S^{1},
$$

both with the standard complex structure $i$. This decomposes $\dot{\Sigma}$ in the form

$$
\dot{\Sigma} \cong \Sigma_{0} \cup\left(\bigcup_{z \in \Gamma^{ \pm}} Z_{ \pm}\right),
$$

where $\Sigma_{0}$ is a compact surface with boundary. We now define the compactified surface $\bar{\Sigma}$ by adding "circles at infinity," which means replacing each $Z_{ \pm}$with $\bar{Z}_{ \pm}$, where

$$
\bar{Z}_{+}=[0, \infty] \times S^{1}, \quad \bar{Z}_{-}=[-\infty, 0] \times S^{1} .
$$

Denote the components of $\partial \bar{\Sigma}$ by $\delta_{z} \cong\{ \pm \infty\} \times S^{1}$ for each $z \in \Gamma^{ \pm}$. We shall not place a smooth structure on $\bar{\Sigma}$. It is naturally a compact topological manifold with boundary, where the interior $\dot{\Sigma} \subset \bar{\Sigma}$ and the boundary components $\delta_{z}$ all have natural smooth structures; in fact the latter have natural identifications with $S^{1}$ up to translation, and one can show that none of this structure depends on the choices.

The symplectization $W:=\mathbb{R} \times M$ also has a natural compactification $\bar{W}:=[-\infty, \infty] \times$ $M$, which we again regard as a topological manifold with boundary, on which the interior and the boundary separately have natural smooth structures. It is then convenient to observe that any finite energy surface $\tilde{u}: \dot{\Sigma} \rightarrow W$ extends naturally to a continuous map $\bar{u}: \bar{\Sigma} \rightarrow \bar{W}$, whose restriction to each $\delta_{z}$ gives an admissible parameterization of the corresponding orbit $P_{z} \subset\{ \pm \infty\} \times M$. 
We say that a sequence $C_{k} \in \mathcal{M}_{\widetilde{J}}$ converges to $C \in \mathcal{M}_{\widetilde{J}}$ if for sufficiently large $k$ there exist representatives $\left(\Sigma, j_{k}, \Gamma, \tilde{u}_{k}\right) \in C_{k}$ and $(\Sigma, j, \Gamma, \tilde{u}) \in C$ such that

(1) $j_{k} \rightarrow j$ in $C^{\infty}(\Sigma)$,

(2) $\tilde{u}_{k} \rightarrow \tilde{u}$ in $C_{\mathrm{loc}}^{\infty}(\dot{\Sigma}, W)$,

(3) $\bar{u}_{k} \rightarrow \bar{u}$ in $C^{0}(\bar{\Sigma}, \bar{W})$.

This defines the topology on the moduli space $\mathcal{M}_{\widetilde{J}}$.

For any finite energy surface $\tilde{u}: \dot{\Sigma} \rightarrow \mathbb{R} \times M$ with Morse-Bott asymptotic orbits, define the index of $\tilde{u}$ by

$$
\operatorname{ind}(\tilde{u})=\mu_{\mathrm{CZ}}(\tilde{u})-\chi(\dot{\Sigma}) .
$$

This is the Fredholm index of the linearized normal Cauchy-Riemann operator $\mathbf{L}_{\tilde{u}}$, which is explained in Hofer-Wysocki-Zehnder [13] for the nondegenerate case and Wendl [28] in general. We call $\tilde{u}$ regular if the operator $\mathbf{L}_{\tilde{u}}$ is surjective; in this case the implicit function theorem allows us to describe a neighborhood of $\tilde{u}$ in $\mathcal{M}_{\tilde{J}}$ as a smooth manifold of dimension ind $(\tilde{u})$. In the general Morse-Bott case there is a stronger notion of regularity: suppose $\tilde{u}$ has a puncture $z \in \Gamma$ at which the asymptotic orbit belongs to a Morse-Bott torus $N \subset M$, and let $\mathcal{U}_{\widetilde{u}} \subset \mathcal{M}_{\widetilde{J}}$ denote a connected open neighborhood of $\tilde{u}$. We can assume without loss of generality that all curves in $\mathcal{U}_{\tilde{u}}$ are parameterized on the same domain $\Sigma$ with the puncture $z \in \Gamma \subset \Sigma$ in a fixed position. The Reeb flow along $N$ defines an $S^{1}$-action so that $N / S^{1}$ is a circle, and there is then a natural evaluation map

$$
\mathrm{ev}_{z}: \mathcal{U}_{\widetilde{u}} \rightarrow N / S^{1}
$$

defined by assigning to any curve in $\mathcal{U}_{\tilde{u}}$ its asymptotic orbit $P_{z} \subset N$. We say that $\tilde{u}$ is strongly regular if it is regular and for every $z \in \Gamma$ where $P_{z}$ is degenerate, $\mathrm{ev}_{z}$ has a surjective linearization at $\tilde{u}$.

Definition 1.6 A finite energy foliation $\mathcal{F}$ is called stable if $\mathcal{P}_{\mathcal{F}}$ is a finite union of nondegenerate Reeb orbits, and every leaf $F \in \mathcal{F}$ is parameterized by a regular finite energy surface $\tilde{u}_{0} \in \mathcal{M}_{\widetilde{J}}$ such that all other curves $\tilde{u} \in \mathcal{M}_{\widetilde{J}}$ near $\tilde{u}_{0}$ also parameterize leaves of $\mathcal{F}$.

We say that $\mathcal{F}$ is a stable foliation of Morse-Bott type if $\mathcal{P}_{\mathcal{F}}$ is a finite union of nondegenerate Reeb orbits and Morse-Bott tori, and each leaf is a strongly regular finite energy surface whose neighbors in $\mathcal{M}_{\widetilde{J}}$ are also leaves of $\mathcal{F}$. 
Observe that leaves of stable finite energy foliations can only have index 0,1 or 2 . The index 0 leaves are precisely the orbit cylinders, while index 1 leaves are called rigid surfaces, because they project to isolated leaves in the foliation of $M \backslash \mathcal{P}_{\mathcal{F}}$. Index 2 leaves come in $\mathbb{R}$-invariant 2-parameter families, which project to 1 -parameter families in $M \backslash \mathcal{P}_{\mathcal{F}}$. In the Morse-Bott case, orbit cylinders can also have index 1, projecting to $M$ as 1 -parameter families moving along Morse-Bott tori.

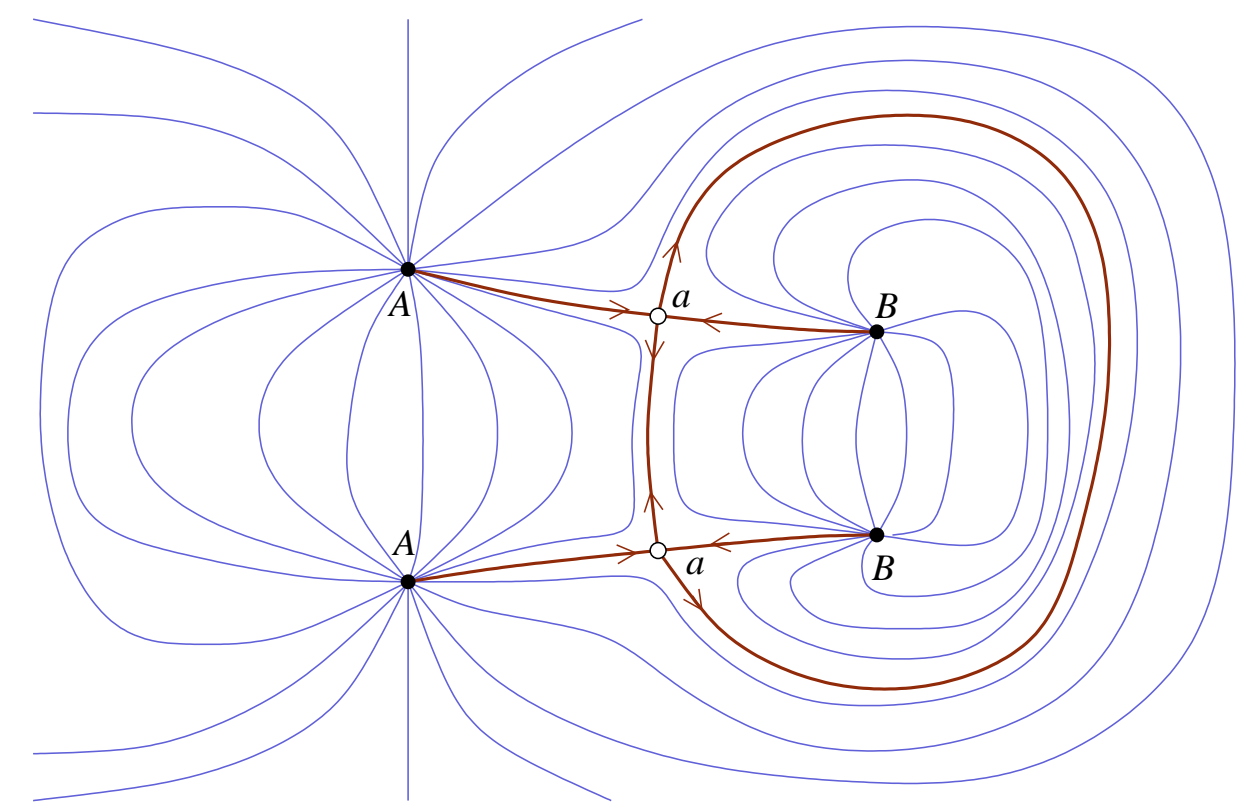

Figure 1: A cross section of a stable finite energy foliation on $S^{3}=\mathbb{R}^{3} \cup$ $\{\infty\}$, with three asymptotic orbits cutting transversely through the page. The hyperbolic orbit $a$ is the limit of two rigid planes, and is connected to two elliptic orbits $A$ and $B$ by rigid cylinders. All other leaves are index 2 planes asymptotic to $A$ or $B$. Arrows represent the signs of the punctures at $a$ : a puncture is positive/negative if the arrow points away from/toward the orbit.

\subsection{Outline of the proof}

The surgery construction involves two main technical ingredients. The first is the Fredholm and intersection theory for the mixed boundary value problem considered in Wendl [28], which we review in Section 2. The crucial point is to observe that embedded index 2 curves with certain properties are always regular, and give rise to nonintersecting 2-parameter families of embedded curves, which project to 1-parameter families of embeddings in $M$. 
The second main ingredient is a compactness argument: this is explained in Section 3 and constitutes the bulk of the technical work in this paper. While we borrow certain ideas from the compactness theorems of Symplectic Field Theory (Bourgeois et al [3]), those results cannot be applied and are in fact not true in our setup, because we make only very weak nondegeneracy assumptions on our data. This is necessary in order to accommodate nontrivial homotopies of the data, but it allows potentially quite strange asymptotic behavior for holomorphic curves. Thus in our situation, the moduli space generally has no natural compactification-yet we'll find that the particular spaces of interest encounter topological obstructions to noncompactness, which are peculiar to the low-dimensional setting. In this way, our arguments are quite different from those in [3]. This is illustrated by the example in Figure 2. Here we consider the degeneration of a sequence of finite energy planes $\widetilde{u}_{k}$ in $\mathbb{R} \times S^{3}$, all asymptotic to the same simply covered orbit $P_{\infty} \subset S^{3}$ and not intersecting it. Without assuming that the Reeb vector field is nondegenerate, it is sometimes possible to show that any other closed orbit $P \subset S^{3}$ must be nontrivially linked with $P_{\infty}$. Then if a plane bubbles off as in the picture, its asymptotic limit $P^{\prime}$ must be linked with $P_{\infty}$, implying that the new plane intersects $P_{\infty}$. But then $P_{\infty}$ must also intersect $\tilde{u}_{k}$ for sufficiently large $k$, giving a contradiction. Some more elaborate variations on this argument will be used repeatedly in Section 3.

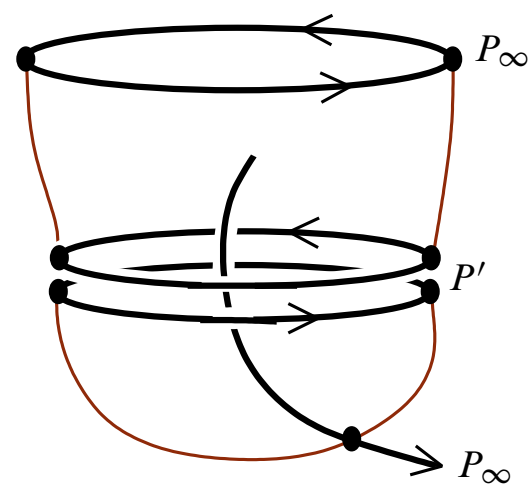

Figure 2: A finite energy plane bubbles off and produces an illegal intersection with the asymptotic limit $P_{\infty}$. This gives a topological obstruction to noncompactness.

The main result is then proved in Section 4. Starting with a stable finite energy foliation on the tight three-sphere (an open book decomposition with one nondegenerate binding orbit), we perform a combination of rational Dehn surgery and Lutz twists along a transverse link $K \subset S^{3}$ and show that the resulting contact manifold also admits a 
stable foliation, now of Morse-Bott type. The topological preliminaries on surgery and Lutz twists are explained in Section 4.1. In Section 4.2, we tackle the easiest step in the foliation construction, finding families of holomorphic curves to fill the solid tori that are glued in by surgery. This is done by explicitly solving the nonlinear Cauchy-Riemann equation on $S^{1} \times B^{2} \subset S^{1} \times \mathbb{R}^{2}$, with rotationally symmetric contact forms and complex structures.

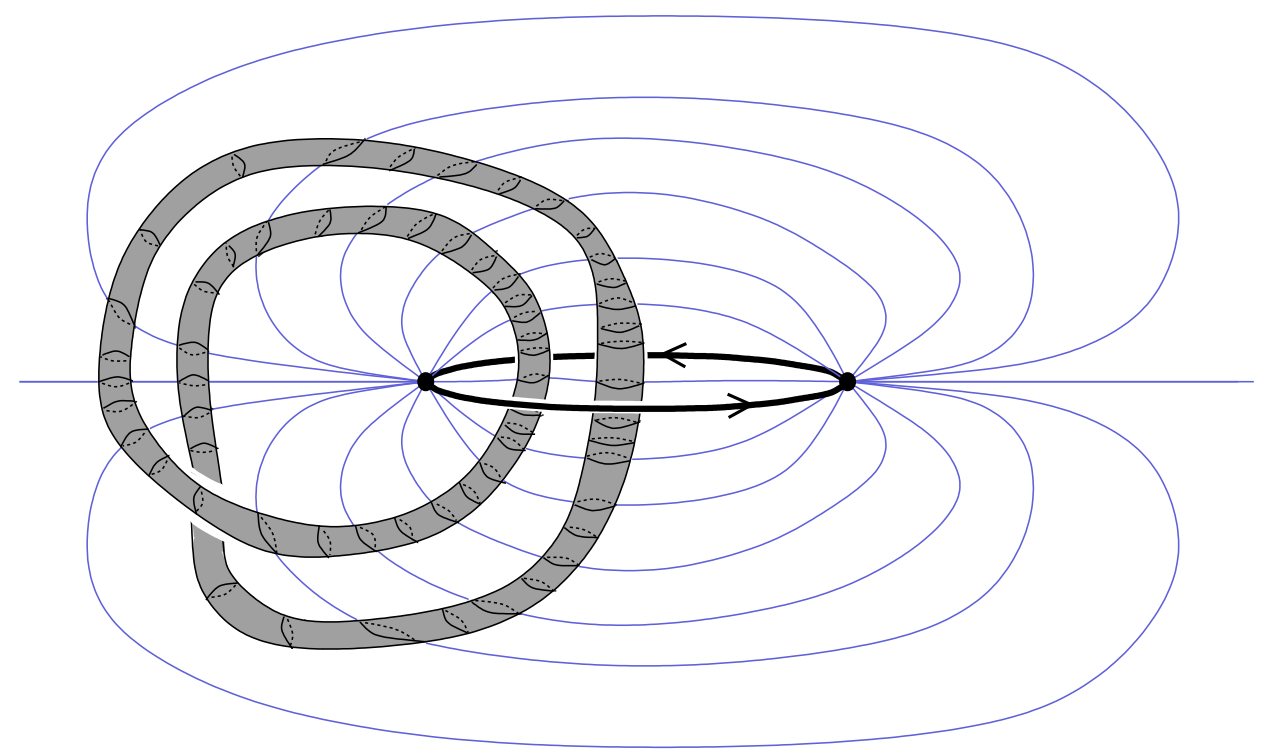

Figure 3: The stable Morse-Bott finite energy foliation obtained from an open book decomposition of $S^{3}$ after surgery along a transverse knot $K$, linked twice with the binding orbit. Each leaf in the region outside the surgery has three punctures: one at the original binding orbit, and two at Morse-Bott orbits along the torus around $K$.

The technical background of Section 2 and Section 3 is then applied in Section 4.3 to change a given open book decomposition of $S^{3}$ (which can also be constructed explicitly) into a stable Morse-Bott foliation in the complement of a transverse link neighborhood. The key is to remove a collection of disks from each page of the open book, obtaining holomorphic curves with boundary, which satisfy a problem of the type considered in Section 2, with images avoiding the region inside a set of small tori. Thus we are now free to perform surgery and Lutz twists inside these tori without killing the holomorphic curves outside; the region inside can afterwards be filled in by the explicitly constructed curves from Section 4.2. 
In principle, the curves with boundary filling the region outside the tori can be homotoped as the contact form is twisted, so that in the limit, all boundary components degenerate to punctures, giving rise to finite energy surfaces without boundary. The actual argument is not quite so simple, because the curves obtained by cutting out disks generally satisfy a boundary condition that is totally real but not Lagrangian, thus lacking a priori energy bounds. This problem does not appear to be solvable for all curves at once, but we can deal with a single curve in the case where each component of $K$ is only singly linked with the binding orbit: this makes it possible to construct the totally real surface in $\mathbb{R} \times M$ so that it is equivalent via a diffeomorphism to a Lagrangian surface in the symplectization of $M$ with a stable Hamiltonian structure, ie the generalization of a contact form described in [3]. We can then homotop this back to contact data but keep the Lagrangian boundary condition, and use the implicit function theorem to extend the single curve again to a whole foliation. The only catch is that this trick requires the restrictive assumption that components of $K$ link only once with the binding orbit. We fix this in Section 4.4 by viewing the general case as a branched cover. The foliation can then be lifted to the cover using intersection theory.

\subsection{Discussion}

The result proved here is one step in a program proposed by Hofer to study Reeb dynamics on arbitrary closed contact 3-manifolds via finite energy foliations; this is joint work in progress by the author with Hofer, R Siefring and J Fish. As was shown in Hofer-Wysocki-Zehnder [16], the existence of finite energy foliations on $\left(S^{3}, \xi_{0}\right)$ implies that generic Reeb vector fields in that setting admit either two or infinitely many periodic orbits. The present work does not imply such a result for overtwisted contact manifolds, because we fix a very specific contact form. The next step would therefore be a homotopy argument in which one shows that a foliation of $(M, \lambda, J)$ gives rise to a foliation (or something similar) of $\left(M, f \lambda, J^{\prime}\right)$ for generic positive smooth functions $f$ and complex multiplications $J^{\prime}$. One can then try to extend this to tight contact manifolds by the following trick: any $(M, \xi)$ can be made overtwisted by taking a connected sum of $(M, \xi)$ with $\left(S^{3}, \xi_{\text {ot }}\right)$ for some $\xi_{\text {ot }}$ overtwisted. One would then like to understand what happens to a sequence of foliations on the overtwisted object as one pinches off the overtwisted part. It is known that this program cannot in general lead to a stable finite energy foliation for generic $(M, \lambda, J)$, as there are examples of tight contact manifolds where stable foliations don't exist (cf [28]). Nonetheless, the limits obtained in such spaces from sequences of foliations should be interesting objects, with possible dynamical implications.

A related program is of a more topological nature: the author proposed in [29, Chapter

6] an equivalence relation for stable finite energy foliations, called concordance, which 
is defined by the existence of stable holomorphic foliations on cylindrical symplectic cobordisms. The goal would then be to classify all foliations for a given $(M, \xi)$ up to concordance. Conjecturally, two concordance classes can be distinguished by an invariant $H C_{*}(\mathcal{F})$, which is a version of contact homology (or more generally, symplectic field theory as in Eliashberg-Givental-Hofer [5]) that counts the orbits and rigid surfaces in the foliation. In this framework, the constructions of the present paper show that every overtwisted $(M, \xi)$ admits a foliation $\mathcal{F}$ for which $H C_{*}(\mathcal{F})$ is trivial. As suggested however by an example in [29], this is not true for all foliations on overtwisted contact manifolds.

We should mention the related work of $\mathrm{C}$ Abbas [1], which uses the open book decompositions of Giroux $[9 ; 8]$ to produce (in the planar case) open book decompositions with pseudoholomorphic pages. Due to Etnyre's result that all overtwisted contact structures are planar [6], this also produces a finite energy foliation for all overtwisted contact manifolds. The two constructions are however quite different, eg ours is not an open book decomposition, and the foliations of Abbas appear to have nontrivial contact homology in the sense described above, suggesting that they are not equivalent to ours via concordance.

Acknowledgements Much of the material covered here appeared originally-in more detailed and sometimes more complicated forms — in my Ph.D. thesis [29], and I thank Helmut Hofer for many valuable suggestions and insights. I'm grateful also to Denis Auroux, Kai Cieliebak, Yasha Eliashberg, Brett Parker, Richard Siefring and Katrin Wehrheim for helpful conversations.

This research was partially supported by NSF grants DMS-0102298 and DMS-0603500.

\section{A mixed boundary value problem}

In this section we review the basic facts about the boundary value problem considered in [28], referring to that paper for all proofs.

Let $M$ be a closed, oriented 3-manifold. A stable Hamiltonian structure on $M$ is a tuple $\mathcal{H}=(\xi, X, \omega, J)$ where

- $\xi$ is a smooth cooriented 2-plane distribution on $M$,

- $\omega$ is a smooth closed 2-form on $M$ which restricts to a symplectic structure on the vector bundle $\xi \rightarrow M$,

- $X$ is a smooth vector field which is transverse to $\xi$, satisfies $\omega(X, \cdot) \equiv 0$, and whose flow preserves $\xi$, 
- $J$ is a smooth complex structure on the bundle $\xi \rightarrow M$, compatible with $\omega$ in the sense that $\omega(\cdot, J \cdot)$ defines a bundle metric.

It follows from these definitions that the flow of $X$ also preserves the symplectic structure defined by $\omega$ on $\xi$, and the special 1 -form $\lambda$ associated to $\xi$ and $X$ by the conditions

$$
\lambda(X) \equiv 1, \quad \operatorname{ker} \lambda \equiv \xi
$$

satisfies $d \lambda(X, \cdot) \equiv 0$.

Example 2.1 Given a contact form $\lambda$ on $M$ with contact structure $\xi=\operatorname{ker} \lambda$, Reeb vector field $X$ and admissible complex multiplication $J$, the data $\mathcal{H}=(\xi, X, d \lambda, J)$ define a stable Hamiltonian structure.

The stable Hamiltonian structure of Example 2.1 is referred to as the contact case. We will define and use a non-contact example in Section 4.3.

An $\mathbb{R}$-invariant almost complex structure $\tilde{J}$ on $\mathbb{R} \times M$ is associated to any $\mathcal{H}=$ $(\xi, X, \omega, J)$ by defining $\widetilde{J} \partial_{a}=X$ and $\widetilde{J} v=J v$ for $v \in \xi$, where again $a$ denotes the coordinate on the $\mathbb{R}$-factor and $\partial_{a}$ is the unit vector in the $\mathbb{R}$-direction. Since $\left.d \lambda\right|_{\xi}$ may now be degenerate, we generalize the definition of energy for pseudoholomorphic curves $\tilde{u}=(a, u):(\Sigma, j) \rightarrow(\mathbb{R} \times M, \widetilde{J})$ by

$$
E(\widetilde{u})=E_{\omega}(\widetilde{u})+E_{\lambda}(\widetilde{u}),
$$

where

$$
E_{\omega}(\widetilde{u})=\int_{\Sigma} u^{*} \omega
$$

is the so-called $\omega$-energy and

$$
E_{\lambda}(\widetilde{u})=\sup _{\varphi \in \mathcal{T}} \int_{\Sigma} \tilde{u}^{*}(d \varphi \wedge \lambda),
$$

with $\mathcal{T}$ defined as in (1). In the contact case this definition is equivalent to (1), in the sense that uniform bounds on one imply uniform bounds on the other. Punctured $\widetilde{J}_{-}$ holomorphic curves with finite energy in this generalized sense also have asymptotically cylindrical behavior near punctures, the same as in Proposition 1.2. The next result, which follows from arguments in (Hofer-Wysocki-Zehnder [11; 14] Siefring [26]), gives a more precise and useful statement. Recall from (5) the definition of the positive and negative half-cylinders $Z_{ \pm}$.

Proposition 2.2 Let $\mathcal{H}=(\xi, X, \omega, J)$ be a stable Hamiltonian structure on $M$ with associated almost complex structure $\widetilde{J}$, and choose a metric on $M$. Suppose $\widetilde{u}=$ 
$(a, u): Z_{ \pm} \rightarrow \mathbb{R} \times M$ is a finite energy $\widetilde{J}$-holomorphic map asymptotic (with sign corresponding to the choice of $Z_{+}$or $Z_{-}$) to a Morse-Bott orbit $P \subset M$ with admissible parameterization $\mathbf{x}: S^{1} \rightarrow M$. There is then a smooth map $h: Z_{ \pm} \rightarrow \mathbf{x}^{*} \xi$ with $h(s, t) \in \xi_{\mathbf{x}(t)}$ such that, up to translation by constants in $s$ and $t, u(s, t)=$ $\exp _{\mathbf{x}(t)} h(s, t)$ for $|s|$ sufficiently large. Moreover, either $h(s, t) \equiv 0$ or it satisfies the formula

$$
h(s, t)=e^{\mu s}(\eta(t)+r(s, t))
$$

where $\eta \in \Gamma\left(\mathbf{x}^{*} \xi\right)$ is an eigenfunction of the asymptotic operator $\mathbf{A}_{\mathbf{x}}$ in (3), $\mu \neq 0$ is the corresponding eigenvalue, whose sign is opposite that of the puncture, and $r(s, t) \rightarrow 0$ uniformly in $t$ as $s \rightarrow \pm \infty$.

Definition 2.3 The section $e \in \Gamma\left(\mathbf{x}^{*} \xi\right)$ appearing in (7) is called the asymptotic eigenfunction of $\tilde{u}$ at the puncture; it is well defined up to a positive multiple. Note that $e(t)$ is never zero, so given a trivialization $\Phi$ of $\mathbf{x}^{*} \xi$, there is a well defined winding

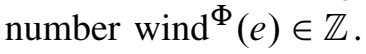

For some integer $m \geq 0$, let $L_{1}, \ldots, L_{m} \subset M$ be a collection of smoothly embedded surfaces which are everywhere tangent to $X$. Choosing smooth functions $g_{j}: L_{j} \rightarrow \mathbb{R}$, the graphs

$$
\widetilde{L}_{j}:=\left\{\left(g_{j}(x), x\right) \in \mathbb{R} \times M \mid x \in L_{j}\right\}
$$

are then totally real submanifolds of $(\mathbb{R} \times M, \widetilde{J})$, and so are their $\mathbb{R}$-translations

$$
\tilde{L}_{j}^{\sigma}:=\left\{\left(g_{j}(x)+\sigma, x\right) \in \mathbb{R} \times M \mid x \in L_{j}\right\}
$$

for every $\sigma \in \mathbb{R}$. Denote $\Lambda=\left(\widetilde{L}_{1}, \ldots, \widetilde{L}_{m}\right)$. Then we define the moduli space

$$
\mathcal{M}_{\mathcal{H}, \Lambda}
$$

to consist of equivalence classes $[(\Sigma, j, \Gamma, \tilde{u})]$ where $(\Sigma, j)$ is a compact Riemann surface with an ordered set of $m$ boundary components

$$
\partial \Sigma=\gamma_{1} \cup \cdots \cup \gamma_{m}
$$

$\Gamma \subset$ int $\Sigma$ is a finite ordered set of interior points giving rise to the punctured Riemann surface $\dot{\Sigma}=\Sigma \backslash \Gamma$ with boundary $\partial \dot{\Sigma}=\partial \Sigma$, and $\widetilde{u}:(\dot{\Sigma}, j) \rightarrow(\mathbb{R} \times M, \widetilde{J})$ is a pseudoholomorphic map with $E(\widetilde{u})<\infty$ and satisfying the following boundary condition:

For each component $\gamma_{j} \subset \partial \Sigma$ there exists a number $\sigma_{j} \in \mathbb{R}$ such that $\tilde{u}\left(\gamma_{j}\right) \subset \widetilde{L}_{j}^{\sigma_{j}}$. 
As before, we will abuse notation and write $\tilde{u} \in \mathcal{M}_{\mathcal{H}, \Lambda}$ whenever convenient. Equivalence in $\mathcal{M}_{\mathcal{H}, \Lambda}$ is defined by biholomorphic maps that preserve the ordering of both the punctures and the boundary components, and the definition of convergence used in Section 1.1 to topologize $\mathcal{M}_{\tilde{J}}$ also naturally gives a topology on $\mathcal{M}_{\mathcal{H}, \Lambda}$. Note that in the case where $\mathcal{H}$ is contact and $m=0$ (ie $\partial \Sigma=\varnothing$ ), $\mathcal{M}_{\mathcal{H}, \Lambda}$ is simply $\mathcal{M}_{\tilde{J}}$, the space of $\widetilde{J}$-holomorphic finite energy surfaces. Observe also that $\mathcal{M}_{\mathcal{H}, \Lambda}$ admits a natural $\mathbb{R}$-action

$$
\mathbb{R} \times \mathcal{M}_{\mathcal{H}, \Lambda} \rightarrow \mathcal{M}_{\mathcal{H}, \Lambda}:(\sigma,(a, u)) \mapsto \tilde{u}^{\sigma}:=(a+\sigma, u)
$$

The Conley-Zehnder index generalizes to $\tilde{u}=(a, u) \in \mathcal{M}_{\mathcal{H}, \Lambda}$ as follows. For each puncture $z \in \Gamma$, let $P_{z}$ be the corresponding orbit of $X$ approached by $\tilde{u}$. The bundle $\xi$ along $P_{z}$ has a symplectic structure $\omega$, which is preserved by the linearized flow of $X$, thus we can choose a unitary trivialization $\Phi$ and define $\mu_{\mathrm{CZ}}^{\Phi \pm}\left(P_{z}\right)$ as in (4). Now at each component $\gamma_{j} \subset \partial \Sigma, u^{*} \xi$ has a totally real subbundle $\ell \rightarrow \gamma_{j}$ defined for $z \in \gamma_{j}$ by

$$
\ell_{z}=\xi_{u(z)} \cap T_{u(z)} L_{j}
$$

here we exploit the fact that $X$ is tangent to $L_{j}$, hence $\xi$ and $L_{j}$ are transverse. Then if $m+\# \Gamma>0$, we can choose trivializations $\Phi$ at each orbit and each boundary component so that these extend to a global complex trivialization of $u^{*} \xi \rightarrow \dot{\Sigma}$, and define the Maslov index of $\tilde{u}$ as

$$
\mu(\tilde{u})=\sum_{z \in \Gamma^{+}} \mu_{\mathrm{CZ}}^{\Phi-}\left(P_{z}\right)-\sum_{z \in \Gamma^{-}} \mu_{\mathrm{CZ}}^{\Phi+}\left(P_{z}\right)+\sum_{j=1}^{m} \mu^{\Phi}\left(\left.u^{*} \xi\right|_{\gamma_{j}}, \ell\right),
$$

where the last term is a sum of Maslov indices for the loops of totally real subspaces $\ell$ along $\gamma_{j}$ with respect to the complex trivialization $\Phi$. If $m=\# \Gamma=0$, so $\dot{\Sigma}$ is closed, we instead define

$$
\mu(\widetilde{u})=2 c_{1}\left(u^{*} \xi\right)
$$

The Fredholm index of $\tilde{u}$ is then the integer

$$
\operatorname{ind}(\tilde{u})=\mu(\widetilde{u})-\chi(\dot{\Sigma})+m .
$$

As with finite energy surfaces, a neighborhood of $\tilde{u} \in \mathcal{M}_{\mathcal{H}, \Lambda}$ can be described via a linearized normal Cauchy-Riemann operator $\mathbf{L}_{\tilde{u}}$, and the previous definitions of regular and strongly regular carry over immediately.

We now collect some useful results about the moduli space $\mathcal{M}_{\mathcal{H}, \Lambda}$. The first important observation is that $\tilde{u}=(a, u) \in \mathcal{M}_{\mathcal{H}, \Lambda}$ is never equivalent to its $\mathbb{R}$-translations $\tilde{u}^{\sigma}=(a+\sigma, u)$ for small $\sigma$ unless it is tangent everywhere to $\mathbb{R} \oplus \mathbb{R} X \subset T(\mathbb{R} \times M)$, 
which implies $E_{\omega}(\tilde{u})=0$. Thus when $E_{\omega}(\tilde{u})>0, \tilde{u}$ and $\tilde{u}^{\sigma}$ have only isolated, positive intersections. The infinitesimal version of this statement deals with the zeros of the section

$$
\pi T u: \dot{\Sigma} \rightarrow \operatorname{Hom}_{\mathbb{C}}\left(T \dot{\Sigma}, u^{*} \xi\right),
$$

which composes the tangent map $T u$ with the projection $\pi: T M \rightarrow \xi$ along $X$. Counting these zeros algebraically defines the nonnegative homotopy invariant $\operatorname{wind}_{\pi}(\widetilde{u})$, which is a half-integer in general because zeros at the boundary must be counted with half weight. The map $u: \dot{\Sigma} \rightarrow M$ is immersed if and only if $\operatorname{wind}_{\pi}(\widetilde{u})=0$, and in this case the Cauchy-Riemann equation implies it is also transverse to $X$. Due to Proposition 2.2 and the relations proved in [12] between the spectrum of the asymptotic operator at an orbit and the orbit's Conley-Zehnder index, $\operatorname{wind}_{\pi}(\widetilde{u})$ is also bounded from above.

Theorem 2.4 For any $\tilde{u} \in \mathcal{M}_{\mathcal{H}, \Lambda}$ with $E_{\omega}(\tilde{u})>0$,

$$
0 \leq 2 \operatorname{wind}_{\pi}(\tilde{u}) \leq \operatorname{ind}(\widetilde{u})-2+2 g+\# \Gamma_{0},
$$

where $g$ denotes the genus of $\Sigma$ and $\Gamma_{0}$ is the set of $z \in \Gamma^{ \pm}$for which $\mu_{C Z}^{\Phi \mp}\left(P_{z}\right)$ is even (for any trivialization $\Phi$ ).

Nondegenerate elliptic orbits always have odd Conley-Zehnder index, thus punctures at such orbits never belong to $\Gamma_{0}$. The next lemma gives a useful criterion in the degenerate Morse-Bott case. It follows, for instance, that $z \notin \Gamma_{0}$ if $P_{z}$ belongs to a Morse-Bott torus which never intersects the image of $u$.

Lemma 2.5 Suppose $\tilde{u}=(a, u) \in \mathcal{M}_{\mathcal{H}, \Lambda}$ is asymptotic at $z \in \Gamma^{ \pm}$to an orbit $P_{z}$ belonging to a Morse-Bott torus $N_{z} \subset M$. Let $\Phi_{0}$ be the natural trivialization of $\xi$ along $P_{z}$ determined by the directions tangent to $N_{z}$, and suppose that the asymptotic eigenfunction $e$ of $\tilde{u}$ at $z$ satisfies wind ${ }^{\Phi_{0}}(e)=0$. Then $\mu_{C Z}^{\Phi_{0} \mp}\left(P_{z}\right)= \pm 1$.

Analogously to $\pi T u$, sections in $\operatorname{ker} \mathbf{L}_{\tilde{u}}$ have only positive zeros and the count of these satisfies a similar upper bound. When this bound is zero in particular, we find $\operatorname{dim} \operatorname{ker} \mathbf{L}_{\tilde{u}} \leq 2$, leading to the conclusion in the embedded index 2 case that $\tilde{u}$ is regular without need for any genericity assumption. Moreover, nearby curves in the moduli space can be identified with sections in $\operatorname{ker} \mathbf{L}_{\tilde{u}}$ which have no zeros, and therefore the nearby curves have no intersections except possibly near infinity. The latter can be excluded a priori in the situation of interest to us here, and we obtain the following special case of a result in [28].

Theorem 2.6 Suppose $\tilde{u}=(a, u): \dot{\Sigma} \rightarrow \mathbb{R} \times M$ represents an element of $\mathcal{M}_{\mathcal{H}, \Lambda}$ and has the following properties: 
(1) $\tilde{u}$ is embedded,

(2) $u$ is injective or $\partial \Sigma=\varnothing$,

(3) all asymptotic orbits $P_{z}$ for $z \in \Gamma$ are Morse-Bott, simply covered and geometrically distinct from one another,

(4) $\operatorname{ind}(\widetilde{u})=2$,

(5) $\Sigma$ has genus 0 and

(6) for each $z \in \Gamma^{ \pm}, \mu_{C Z}^{\Phi \mp}\left(P_{z}\right)$ is odd (for any trivialization $\Phi$ ).

Then $\tilde{u}$ is strongly regular and a neighborhood of $\tilde{u}$ in $\mathcal{M}_{\mathcal{H}, \Lambda}$ has naturally the structure of a smooth 2-dimensional manifold. In fact, there exists an embedding

$$
\begin{aligned}
\mathbb{R} \times(-1,1) \times \dot{\Sigma} & \stackrel{\widetilde{F}}{\longrightarrow} \mathbb{R} \times M \\
(\sigma, \tau, z) & \longmapsto\left(a_{\tau}(z)+\sigma, u_{\tau}(z)\right)
\end{aligned}
$$

such that the following hold.

(i) For $\sigma \in \mathbb{R}$ and $\tau \in(-1,1)$, the embeddings $\tilde{u}_{(\sigma, \tau)}=\tilde{F}(\sigma, \tau, \cdot): \dot{\Sigma} \rightarrow \mathbb{R} \times M$ parameterize elements of $\mathcal{M}_{\mathcal{H}, \Lambda}$, and $\tilde{u}_{(0,0)}=\tilde{u}$.

(ii) The map $F(\tau, z)=u_{\tau}(z)$ is an embedding $(-1,1) \times \dot{\Sigma} \hookrightarrow M$, and its image never intersects any of the orbits $P_{z}$ or Morse-Bott tori $N_{z}$. In particular the maps $u_{\tau}: \dot{\Sigma} \rightarrow M$ are embedded for each $\tau \in(-1,1)$, with mutually disjoint images which do not intersect their asymptotic limits.

(iii) If $P_{z}$ belongs to a Morse-Bott torus $N_{z}$, then $u_{\tau}$ and $u_{\tau^{\prime}}$ are asymptotic at $z$ to distinct orbits in $N_{z}$ whenever $\tau \neq \tau^{\prime}$.

(iv) Every $\tilde{u}^{\prime}$ sufficiently close to $\tilde{u}$ in $\mathcal{M}_{\mathcal{H}, \Lambda}$ is parameterized by $\tilde{u}_{(\sigma, \tau)}$ for some unique $\sigma \in \mathbb{R}, \tau \in(-1,1)$.

From this and the smoothness of the nonlinear normal Cauchy-Riemann operator defined in [28], we obtain the following deformation result.

Theorem 2.7 Suppose $\tilde{u} \in \mathcal{M}_{\mathcal{H}, \Lambda}$ is as in Theorem 2.6, and

$$
\mathcal{H}_{r}=\left(\xi_{r}, X_{r}, \omega_{r}, J_{r}\right), \quad r \in(-1,1)
$$

is a smooth 1-parameter family of stable Hamiltonian structures such that $\mathcal{H}_{0}=\mathcal{H}$, and for each $r \in(-1,1)$, all of the orbits $P_{z}$ and Morse-Bott tori $N_{z}$ remain Morse-Bott orbits/tori with respect to $X_{r}$, while the surfaces $L_{1}, \ldots, L_{m}$ remain tangent to $X_{r}$. 
Then there exists a number $\epsilon \in(0,1]$ and a smooth 1 -parameter family of maps

$$
\widetilde{F}_{r}: \mathbb{R} \times(-1,1) \times \dot{\Sigma} \rightarrow \mathbb{R} \times M, \quad r \in(-\epsilon, \epsilon)
$$

such that $\widetilde{F}_{0}(0,0, \cdot)=\tilde{u}$ and each map $\widetilde{F}_{r}$ has the properties of $\widetilde{F}$ in Theorem 2.6 with respect to the moduli space $\mathcal{M}_{\mathcal{H}_{r}, \Lambda}$.

These local perturbation theorems start from the assumption that $\tilde{u}=(a, u)$ is embedded, and usually also that $u$ is injective. To study foliations, we need to know that such conditions are preserved under convergence to limits. One needs therefore a version of positivity of intersections for holomorphic curves with boundary: the crucial observation is that such a result holds whenever one can guarantee that all boundary intersections are "one-sided" (cf Ye [30]). This is easy to show under the conditions of Theorem 2.6, where the assumptions guarantee that $\operatorname{wind}_{\pi}(\widetilde{u})=0$, implying $u$ is transverse to $X$ and thus $\tilde{u}$ is transverse to the hypersurfaces $\mathbb{R} \times L_{j}$.

Theorem 2.8 Suppose $\mathcal{H}_{k}=\left(\xi_{k}, X_{k}, \omega_{k}, J_{k}\right)$ is a sequence of stable Hamiltonian structures converging in $C^{\infty}(M)$ to $\mathcal{H}=(\xi, X, \omega, J)$, such that $X_{k}$ is tangent to the surfaces $L_{1}, \ldots, L_{m}$ for all $k$, and $\tilde{u}_{k}=\left(a_{k}, u_{k}\right): \dot{\Sigma} \rightarrow \mathbb{R} \times M$ is a sequence of curves in $\mathcal{M}_{\mathcal{H}_{k}, \Lambda}$ such that for all $k, u_{k}$ is embedded and intersects each $L_{j}$ only at $\partial \Sigma$. Then if $\tilde{u}_{k}$ converges in $C_{\text {loc }}^{\infty}$ to a somewhere injective curve $\tilde{u}=(a, u) \in \mathcal{M}_{\mathcal{H}, \Lambda}$ with $E_{\omega}(\widetilde{u})>0$ and $\operatorname{wind}_{\pi}(\widetilde{u})=0, u: \dot{\Sigma} \rightarrow M$ is embedded.

Moreover, suppose $\tilde{u}_{k}=\left(a_{k}, u_{k}\right), \widetilde{v}_{k}=\left(b_{k}, v_{k}\right) \in \mathcal{M}_{\mathcal{H}_{k}, \Lambda}$ are sequences such that $u_{k}$ and $v_{k}$ both satisfy the conditions above, and never intersect each other. Then if $\tilde{u}_{k} \rightarrow \tilde{u}=(a, u)$ and $\widetilde{v}_{k} \rightarrow \widetilde{v}=(b, v)$ in $C_{\text {loc }}^{\infty}$ with $E_{\omega}(\widetilde{u})$ and $E_{\omega}(\widetilde{v})$ both positive and $\operatorname{wind}_{\pi}(\widetilde{u})=\operatorname{wind}_{\pi}(\widetilde{v})=0$, the images of $u$ and $v$ are either disjoint or identical.

\section{Compactness}

\subsection{The setup}

For any pair of oriented knots $\gamma$ and $\gamma^{\prime} \subset S^{3}$, denote their linking number by $\operatorname{lk}\left(\gamma, \gamma^{\prime}\right) \in$ $\mathbb{Z}$. Let $P_{\infty} \subset S^{3}$ be an oriented knot, and $K=K_{1} \cup \cdots \cup K_{m} \subset S^{3} \backslash P_{\infty}$ an oriented link whose components satisfy $\operatorname{lk}\left(P_{\infty}, K_{j}\right)>0$. Each knot $K_{j}$ is the center of some solid torus $N_{j} \subset S^{3}$; we assume that these solid tori are pairwise disjoint and that $N:=N_{1} \cup \cdots \cup N_{m} \subset S^{3}$ is disjoint from $P_{\infty}$. Denote $\partial N_{j}=L_{j}$ and $M=S^{3} \backslash($ int $N)$, so the oriented boundary of $M$ is $\partial M=-\bigcup_{j} L_{j}$. Let $\mathcal{H}_{k}=\left(\xi_{k}, X_{k}, \omega_{k}, J_{k}\right)$ be a sequence of stable Hamiltonian structures on $M$ which converge in $C^{\infty}(M)$ to a stable Hamiltonian structure $\mathcal{H}_{\infty}=\left(\xi_{\infty}, X_{\infty}, \omega_{\infty}, J_{\infty}\right)$ and have the following properties for all $k \leq \infty$. 
(1) $P_{\infty}$ is a nondegenerate periodic orbit of $X_{k}$.

(2) Any other periodic orbit $P \subset M \backslash P_{\infty}$ of $X_{k}$ satisfies $\operatorname{lk}\left(P, P_{\infty}\right) \neq 0$.

(3) $X_{k}$ is tangent to each torus $L_{j}$.

(4) There are trivializations $\Phi_{k}$ of $\left.\xi_{k}\right|_{M}$ such that $\mu_{\mathrm{CZ}}^{\Phi_{k}}\left(P_{\infty}\right)=3$ and, if $\gamma \subset L_{j}$ is a positively oriented meridian, $\operatorname{wind}_{\gamma}^{\Phi_{k}}\left(T L_{j} \cap \xi_{k}\right)=1$.

The stable Hamiltonian structures $\mathcal{H}_{k}$ define $\mathbb{R}$-invariant almost complex structures $\widetilde{J}_{k}$ on $\mathbb{R} \times M$, for which the surfaces

$$
\tilde{L}_{j}:=\{0\} \times L_{j}
$$

are totally real submanifolds. We then consider a sequence of embedded $\widetilde{J}_{k}$-holomorphic curves

$$
\tilde{u}_{k}=\left(a_{k}, u_{k}\right):\left(\dot{\Sigma}, j_{k}\right) \rightarrow\left(\mathbb{R} \times M, \widetilde{J}_{k}\right),
$$

where

$$
\Sigma=S^{2} \backslash \bigcup_{j=1}^{m} \mathcal{D}_{j}
$$

for some set of pairwise disjoint open disks $\mathcal{D}_{j} \subset \mathbb{C}, j_{k}$ is an arbitrary sequence of smooth complex structures on $\Sigma$ and $\dot{\Sigma}=\Sigma \backslash\{\infty\}$. Assume each $\tilde{u}_{k}$ has finite energy with respect to $\mathcal{H}_{k}$, is positively asymptotic at $\infty$ to the orbit $P_{\infty}$, and satisfies the boundary condition

$$
\tilde{u}_{k}\left(\gamma_{j}\right) \subset\{\text { const }\} \times L_{j}
$$

for $\gamma_{j}=\partial \mathcal{D}_{j}$, where the constant in the $\mathbb{R}$-factor is arbitrary and independent for each component. Thus $\tilde{u}_{k} \in \mathcal{M}_{\mathcal{H}_{k}, \Lambda}$, where $\Lambda=\left(\widetilde{L}_{1}, \ldots, \widetilde{L}_{m}\right)$. We assume also that the maps $u_{k}: \dot{\Sigma} \rightarrow M$ are all injective and have the following topological behavior at the boundary.

For each component $\gamma_{j} \subset \partial \Sigma$, the oriented loop $u_{k}\left(\gamma_{j}\right)$ is homotopic along $L_{j}$ to a negatively oriented meridian, ie $\operatorname{lk}\left(u_{k}\left(\gamma_{j}\right), P_{\infty}\right)=0$ and $\operatorname{lk}\left(u_{k}\left(\gamma_{j}\right), K_{j}\right)=$ -1 .

Remark 3.1 We've implicitly assumed thus far that $L_{i}$ and $L_{j}$ are disjoint when $i \neq j$, but it's convenient also to allow the possibility that $K$ has fewer than $m$ components. As a notational convenience, we will continue to list these components as $K_{1} \cup \cdots \cup K_{m}$, with the understanding that some of the $K_{j}$ (and the corresponding $N_{j}$ and $L_{j}$ ) may be identical. In this case different components of $\partial \Sigma$ may satisfy the same boundary condition.

Lemma 3.2 $\operatorname{ind}\left(\widetilde{u}_{k}\right)=2$. 
Proof Using the global trivialization $\Phi_{k}$, the boundary Maslov index at $\gamma_{j}$ is twice the winding number of $T L_{j} \cap \xi_{k}$ along a negatively oriented meridian; this gives -2 . We then have

$$
\mu\left(\tilde{u}_{k}\right)=\mu_{\mathrm{CZ}}^{\Phi_{k}}\left(P_{\infty}\right)+\sum_{j=1}^{m} \mu_{u_{k}\left(\gamma_{j}\right)}^{\Phi_{k}}\left(u^{*} \xi_{k}, T L_{j} \cap \xi_{k}\right)=3-2 m,
$$

hence by (9),

$$
\operatorname{ind}\left(\tilde{u}_{k}\right)=\mu\left(\tilde{u}_{k}\right)-\chi(\dot{\Sigma})+m=(3-2 m)-(1-m)+m=2 .
$$

Applying Theorem 2.6 to $\tilde{u}_{k}$ now yields the following Corollary.

Corollary 3.3 For each $\tilde{u}_{k}=\left(a_{k}, u_{k}\right), u_{k}: \dot{\Sigma} \rightarrow M$ is embedded, transverse to $X_{k}$ and does not intersect $P_{\infty}$. In particular then, $u_{k}$ is transverse to $\partial M$.

Our main goal in the next few subsections will be to prove the following theorem.

Theorem 3.4 (Compactness) There exists a sequence of numbers $c_{k} \in \mathbb{R}$ and diffeomorphisms $\varphi_{k}: \Sigma \rightarrow \Sigma$ which fix $\infty$ and preserve each component of $\partial \Sigma$, such that a subsequence of $\left(a_{k}+c_{k}, u_{k}\right) \circ \varphi_{k}$ converges in $C_{\text {loc }}^{\infty}(\dot{\Sigma})$ to some $\tilde{u}=(a, u) \in \mathcal{M}_{\mathcal{H}_{\infty}, \Lambda}$, and the continuous extensions of these maps over $\bar{\Sigma}$ converge in $C^{0}$. Moreover, $\tilde{u}$ is embedded and $u$ is injective.

A closely related result involves the degeneration of such a sequence as $X_{k}$ is twisted to the point where the meridians on $L_{j}$ become periodic orbits. The following will be crucial in passing from holomorphic foliations with boundary to stable finite energy foliations of Morse-Bott type.

Theorem 3.5 (Degeneration) Assume $\tilde{u}_{k}$ and $\mathcal{H}_{k}$ are as described above for all $k<\infty$, but with the following change for $k=\infty$.

Any periodic orbit $P$ of $X_{\infty}$ in (int $\left.M\right) \backslash P_{\infty}$ satisfies $\operatorname{lk}\left(P, P_{\infty}\right) \neq 0$, and each $L_{j} \subset \partial M$ is a Morse-Bott torus with respect to $X_{\infty}$, with orbits $P$ satisfying $\operatorname{lk}\left(P, P_{\infty}\right)=0$ and $\operatorname{lk}\left(P, K_{j}\right)=-1$.

Then there is a finite set $\Gamma^{\prime} \subset \mathbb{C}$ with $\# \Gamma^{\prime}=m$, a sequence of numbers $c_{k} \in \mathbb{R}$ and diffeomorphisms $\varphi_{k}: S^{2} \backslash \Gamma^{\prime} \rightarrow$ int $\Sigma$ that fix $\infty$, such that after passing to a subsequence, $\varphi_{k}^{*} j_{k} \rightarrow i$ in $C_{\mathrm{loc}}^{\infty}\left(S^{2} \backslash \Gamma^{\prime}\right)$ and $\left(a_{k}+c_{k}, u_{k}\right) \circ \varphi_{k}$ converges in $C_{\mathrm{loc}}^{\infty}\left(\mathbb{C} \backslash \Gamma^{\prime}, \mathbb{R} \times M\right)$ to a $\widetilde{J}_{\infty}$-holomorphic finite energy surface

$$
\tilde{u}_{\infty}: S^{2} \backslash\left(\{\infty\} \cup \Gamma^{\prime}\right) \rightarrow \mathbb{R} \times M .
$$


The map $\tilde{u}_{\infty}=\left(a_{\infty}, u_{\infty}\right)$ is embedded, with $u_{\infty}: \mathbb{C} \backslash \Gamma^{\prime} \rightarrow M$ injective and disjoint from $\partial M$, all the punctures are positive, the asymptotic limit at $\infty \in S^{2}$ is $P_{\infty}$, and for each component $\gamma_{j} \subset \partial \Sigma$, there is a corresponding puncture $z_{j} \in \Gamma^{\prime}$ such that the asymptotic limit at $z_{j}$ is a simply covered orbit on $L_{j}$. Moreover for any sequence $\zeta_{k} \in \mathbb{C} \backslash \Gamma^{\prime}$ approaching a puncture $z_{j} \in \Gamma^{\prime}$, we have $u_{k} \circ \varphi_{k}\left(\zeta_{k}\right) \rightarrow L_{j}$.

\subsection{Deligne-Mumford theory with boundary}

Before proving the main compactness results, we briefly review the Deligne-Mumford compactification of the moduli space of Riemann surfaces with boundary and interior marked points. This space can always be identified with a space of symmetric surfaces without boundary, "symmetric" meaning there exists an antiholomorphic involution which is respected by all the data (see Figure 4). A more detailed discussion of this correspondence may be found in [29], and for proofs of the compactness theorem we refer to Seppälä-Sorvali [25] and Hummel [18].

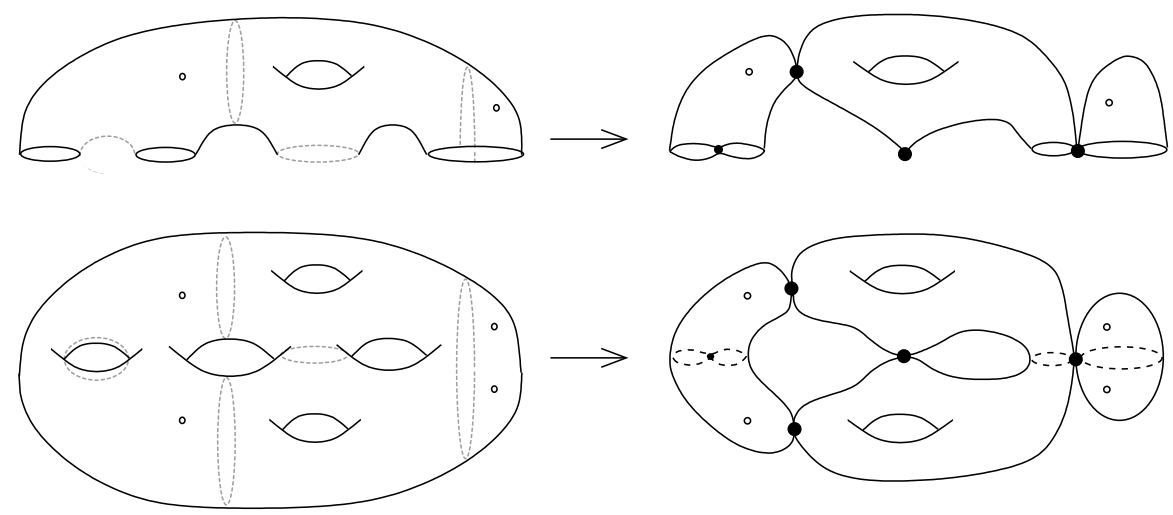

Figure 4: Degeneration of a stable Riemann surface $(\Sigma, j, \Gamma)$ with genus 1 , four boundary components and two interior marked points, together with its symmetric doubled surface. The lightly shaded curves on the left are the geodesic loops and arcs that shrink to zero length in the limit. The right side shows the corresponding singular surface $\widehat{\boldsymbol{\Sigma}}$ and its double after degeneration; $\widehat{\boldsymbol{\Sigma}}$ has one interior double point, two boundary double points and one unpaired node.

Let $(\Sigma, j)$ be a compact connected Riemann surface, possibly with boundary, and let $\Gamma \subset$ int $\Sigma$ be a finite ordered subset. As usual, denote the corresponding punctured surface by $\dot{\Sigma}=\Sigma \backslash \Gamma$. If the Euler characteristic $\chi(\dot{\Sigma})<0$, then we call the triple 
$(\Sigma, j, \Gamma)$ a stable Riemann surface with boundary and interior marked points. The stability condition means

$$
2 g+m+\# \Gamma>2,
$$

where $g$ is the genus of $\Sigma$ and $m$ is the number of boundary components. Equivalently, one can say that $(\Sigma, j, \Gamma)$ is stable if the Riemann surface obtained by doubling $(\dot{\Sigma}, j)$ along its boundary has negative Euler characteristic; this definition has the advantage of also being correct when $\Sigma$ has marked points on the boundary.

It is a standard fact that every Riemann surface without boundary and with negative Euler characteristic admits a unique complete metric that is compatible with the conformal structure and has constant curvature -1 : this is called the Poincaré metric. For a stable Riemann surface $(\Sigma, j, \Gamma)$ with boundary, we define the Poincare metric as the restriction to $\dot{\Sigma}$ of the Poincaré metric on the symmetric double of $(\dot{\Sigma}, j)$. In this way, each component of $\partial \Sigma$ becomes a geodesic.

Denote by $\mathcal{M}_{g, m, p}$ the moduli space of equivalence classes of compact connected Riemann surfaces $(\Sigma, j, \Gamma)$ with genus $g, m \geq 0$ boundary components and $p=\# \Gamma$ interior marked points $\Gamma \subset$ int $\Sigma$. Recall that the points of $\Gamma$ come with an ordering. Equivalence $(\Sigma, j, \Gamma) \sim\left(\Sigma^{\prime}, j^{\prime}, \Gamma^{\prime}\right)$ means that there exists a biholomorphic map $\varphi:(\Sigma, j) \rightarrow\left(\Sigma^{\prime}, j^{\prime}\right)$ that takes $\Gamma$ to $\Gamma^{\prime}$, preserving the ordering. The topology on $\mathcal{M}_{g, m, p}$ is defined by saying that $\left[\left(\Sigma_{k}, j_{k}, \Gamma_{k}\right)\right] \rightarrow[(\Sigma, j, \Gamma)]$ if for sufficiently large $k$ there exist diffeomorphisms $\varphi_{k}: \Sigma \rightarrow \Sigma_{k}$ mapping $\Gamma \rightarrow \Gamma_{k}$ (with the right ordering) and such that $\varphi_{k}^{*} j_{k} \rightarrow j$ in $C^{\infty}$.

A nodal Riemann surface with boundary and interior marked points $\boldsymbol{\Sigma}=(\Sigma, j, \Gamma, \Delta, N)$ consists of a Riemann surface $(\Sigma, j)$ with finitely many connected components $\Sigma=$ $\Sigma_{1} \cup \cdots \cup \Sigma_{q}$, each of which is a compact surface, possibly with boundary. The marked point set $\Gamma$ is a finite ordered set of interior points in $\Sigma$, and $\Delta$ is a set of unordered pairs of points in $\Sigma$,

$$
\Delta=\left\{\left\{z_{1}, z_{1}^{\prime}\right\}, \ldots,\left\{z_{d}, z_{d}^{\prime}\right\}\right\}
$$

called double points. By assumption, the points $z_{1}, z_{1}^{\prime}, \ldots, z_{d}, z_{d}^{\prime}$ are all distinct, and $z_{j} \in \partial \Sigma$ if and only if $z_{j}^{\prime} \in \partial \Sigma$. We will sometimes abuse notation and regard $\Delta$ as a subset of $\Sigma$, rather than a set of pairs. There is also a finite unordered set $N$ of interior points, which we'll call unpaired nodes. We assume the sets $\Gamma, \Delta$ and $N$ are all disjoint. Intuitively, one thinks of $\boldsymbol{\Sigma}$ as the topological space obtained from $\Sigma$ by identifying each pair of double points:

$$
\widehat{\Sigma}=\Sigma /\left\{z_{j} \sim z_{j}^{\prime} \text { for each pair }\left\{z_{j}, z_{j}^{\prime}\right\} \in \Delta\right\} .
$$

The point in $\widehat{\boldsymbol{\Sigma}}$ determined by a given pair of double points $\left\{z_{j}, z_{j}^{\prime}\right\} \in \Delta$ is called a node. We say that $\boldsymbol{\Sigma}$ is connected whenever $\widehat{\boldsymbol{\Sigma}}$ is connected. The connected 
components of $\Sigma$ may be regarded as Riemann surfaces with boundary and marked points $\left(\Sigma_{j},\left.j\right|_{\Sigma_{j}},(\Gamma \cup \Delta \cup N) \cap \Sigma_{j}\right)$, which give rise to punctured surfaces

$$
\dot{\Sigma}_{j}=\Sigma_{j} \backslash\left((\Gamma \cup \Delta \cup N) \cap \Sigma_{j}\right),
$$

having potentially both interior and boundary punctures. We then say that $\Sigma$ is stable if its connected components are all stable; this means each $\dot{\Sigma}_{j}$ has negative Euler characteristic after doubling.

The punctured components $\dot{\Sigma}_{j}$ can be compactified naturally as follows: for an interior puncture $z$, choose holomorphic coordinates identifying $z$ with 0 in the standard unit disk, use the map $(s, t) \mapsto e^{-2 \pi(s+i t)}$ to identify this biholomorphically with the halfcylinder $[0, \infty) \times S^{1}$, and compactify by adding the "circle at infinity" $\delta_{z} \cong\{\infty\} \times S^{1}$. For punctures $z \in \partial \Sigma$, one instead uses the map $(s, t) \mapsto e^{-\pi(s+i t)}$ to identify a punctured upper half-disk with the half strip $[0, \infty) \times[0,1]$, and then adds the "arc at infinity" $\delta_{z} \cong\{\infty\} \times[0,1]$. Doing this for all punctures yields a compact surface with piecewise smooth boundary. Denote by $\bar{\Sigma}_{j}$ the compactification of $\dot{\Sigma}_{j} \cup\left(\Gamma \cap \Sigma_{j}\right)$ obtained by adding such circles for each interior double point and unpaired node, and arcs for each boundary double point in $\Sigma_{j}$.

Given a nodal surface $(\Sigma, j, \Gamma, \Delta, N)$, an asymptotic marker at $z \in \Delta \cap$ int $\Sigma$ is a choice of direction $\mu \in\left(T_{z} \Sigma \backslash\{0\}\right) / \mathbb{R}_{+}$, where $\mathbb{R}_{+}$is the group of positive real numbers, acting by scalar multiplication. A choice of asymptotic markers $r=$ $\left\{\left\{\mu_{1}, \mu_{1}^{\prime}\right\}, \ldots,\left\{\mu_{d}, \mu_{d}^{\prime}\right\}\right\}$ for every pair of interior double points is called a decoration, and we then call $(\Sigma, r)=(\Sigma, j, \Gamma, \Delta, N, r)$ a decorated nodal Riemann surface. For each pair $\left\{z, z^{\prime}\right\} \in \Delta$ with asymptotic markers $\left\{\mu, \mu^{\prime}\right\}$, the conformal structure $j$ determines a natural choice of orientation reversing map

$$
r_{z}:\left(T_{z} \Sigma \backslash\{0\}\right) / \mathbb{R}_{+} \rightarrow\left(T_{z^{\prime}} \Sigma \backslash\{0\}\right) / \mathbb{R}_{+}
$$

such that $r_{z}(\mu)=\mu^{\prime}$, and hence also an orientation reversing diffeomorphism $\bar{r}_{z}: \delta_{z} \rightarrow$ $\delta_{z^{\prime}}$. For boundary pairs $\left\{z, z^{\prime}\right\}$, the boundary determines natural asymptotic markers and thus diffeomorphisms $\bar{r}_{z}$ between the $\operatorname{arcs} \delta_{z}$ and $\delta_{z^{\prime}}$. Then define

$$
\bar{\Sigma}_{r}=\left(\bar{\Sigma}_{1} \sqcup \cdots \sqcup \bar{\Sigma}_{q}\right) /\left\{w \sim \bar{r}_{z}(w) \text { for all } w \in \delta_{z}, z \in \Delta \cup N\right\} .
$$

This is a compact surface with smooth boundary, and is connected if and only if $\widehat{\Sigma}$ is connected. In that case, we define the arithmetic signature of $\boldsymbol{\Sigma}$ to be the pair $(g, m)$ where $g$ is the genus of $\overline{\boldsymbol{\Sigma}}_{r}$ (the arithmetic genus of $\boldsymbol{\Sigma}$ ) and $m$ is the number of connected components of $\partial \overline{\boldsymbol{\Sigma}}_{r}$. We shall denote the union of the special circles and $\operatorname{arcs} \delta_{z}$ for $z \in \Delta \cup N$ by $\Theta_{\Delta, N} \subset \bar{\Sigma}_{r}$. The conformal structure $j$ on $\Sigma$ defines a singular conformal structure $j_{\Sigma}$ on $\bar{\Sigma}_{r}$, which degenerates at $\Theta_{\Delta, N}$. If $\Sigma$ is stable, then there is similarly a "singular Poincaré metric" $h_{\Sigma}$ on $\bar{\Sigma}_{r} \backslash \Gamma$, defined by choosing 
the Poincare metric on each of the punctured components $\dot{\Sigma}_{j}$. This metric degenerates at $\Theta_{\Delta, N}$ as well as at $\Gamma$; in particular the distance from a marked point $z_{0} \in \Gamma$ or a circle or arc $\delta_{z} \subset \Theta_{\Delta, N}$ to any other point in $\overline{\boldsymbol{\Sigma}}_{r}$ is infinite, and the circles and arcs $\delta_{z}$ have length 0 . Observe that in the stable case, $\chi\left(\bar{\Sigma}_{r} \backslash \Gamma\right)<0$, ie a stable nodal surface $(\Sigma, j, \Gamma, \Delta, N)$ with arithmetic signature $(g, m)$ satisfies $2 g+m+\# \Gamma>2$.
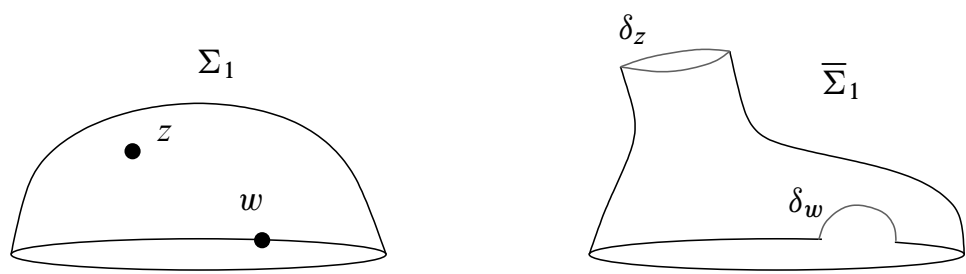

Figure 5: A component $\Sigma_{1}$ with its compactification $\bar{\Sigma}_{1}$. Here there's one interior double point $z \in \Delta \cap$ int $\Sigma_{1}$ and one boundary double point $w \in$ $\Delta \cap \partial \Sigma_{1}$.

Assume $2 g+m+p>2$ and let $\overline{\mathcal{M}}_{g, m, p}$ denote the moduli space of equivalence classes of stable nodal Riemann surfaces $\Sigma=(\Sigma, j, \Gamma, \Delta, N)$ with arithmetic signature $(g, m)$ and $p=\# \Gamma$ interior marked points. We say $(\Sigma, j, \Gamma, \Delta, N) \sim\left(\Sigma^{\prime}, j^{\prime}, \Gamma^{\prime}, \Delta^{\prime}, N^{\prime}\right)$ if there is a biholomorphic map $\varphi:(\Sigma, j) \rightarrow\left(\Sigma^{\prime}, j^{\prime}\right)$ taking $\Gamma$ to $\Gamma^{\prime}$ with the proper ordering, and such that $\varphi(N)=N^{\prime}$ and $\left\{\varphi\left(z_{1}\right), \varphi\left(z_{2}\right)\right\} \in \Delta^{\prime}$ if and only if $\left\{z_{1}, z_{2}\right\} \in \Delta$. There is a natural inclusion $\mathcal{M}_{g, m, p} \hookrightarrow \overline{\mathcal{M}}_{g, m, p}$ defined by assigning to $[(\Sigma, j, \Gamma)]$ an empty set of double points and unpaired nodes.

The topology of $\overline{\mathcal{M}}_{g, m, p}$ is determined by the following notion of convergence.

Definition 3.6 A sequence $\left[\Sigma_{k}\right]=\left[\left(\Sigma_{k}, j_{k}, \Gamma_{k}, \Delta_{k}, N_{k}\right)\right] \in \overline{\mathcal{M}}_{g, m, p}$ converges to $[\Sigma]=[(\Sigma, j, \Gamma, \Delta, N)] \in \overline{\mathcal{M}}_{g, m, p}$ if there are decorations $r_{k}$ for $\boldsymbol{\Sigma}_{k}$ and $r$ for $\boldsymbol{\Sigma}$, and diffeomorphisms $\varphi_{k}: \bar{\Sigma}_{r} \rightarrow\left(\bar{\Sigma}_{k}\right)_{r_{k}}$, for sufficiently large $k$, with the following properties.

(1) $\varphi_{k}$ sends $\Gamma$ to $\Gamma_{k}$, preserving the ordering.

(2) $\varphi_{k}^{*} j_{\boldsymbol{\Sigma}_{k}} \rightarrow j_{\boldsymbol{\Sigma}}$ in $C_{\mathrm{loc}}^{\infty}\left(\overline{\boldsymbol{\Sigma}}_{r} \backslash \Theta_{\Delta, N}\right)$.

(3) $\varphi_{k}^{-1}\left(\Theta_{\Delta_{k}, N_{k}}\right) \subset \Theta_{\Delta, N}$, and all circles in $\varphi_{k}\left(\Theta_{\Delta, N}\right) \backslash \Theta_{\Delta_{k}, N_{k}}$ are closed geodesics for the Poincaré metric $h_{\Sigma_{k}}$ on $\left(\bar{\Sigma}_{k}\right)_{r_{k}}$; similarly all arcs in $\varphi_{k}\left(\Theta_{\Delta, N}\right) \backslash \Theta_{\Delta_{k}, N_{k}}$ are geodesic arcs with endpoints on $\partial\left(\left(\bar{\Sigma}_{k}\right)_{r_{k}}\right)$.

Theorem 3.7 $\overline{\mathcal{M}}_{g, m, p}$ is compact. In particular, any sequence of stable Riemann surfaces $\left(\Sigma_{k}, j_{k}, \Gamma_{k}\right)$ with boundary and interior marked points, having fixed topological type and number of marked points, has a subsequence convergent (in the sense of 
Definition 3.6) to a stable nodal Riemann surface $(\Sigma, j, \Gamma, \Delta, N)$ with boundary and interior marked points.

\subsection{Preparation and removal of singularities}

In this section, fix a closed 3-manifold $M$ with stable Hamiltonian structure $\mathcal{H}=$ $(\xi, X, \omega, J)$ and an embedded surface $L \subset M$ tangent to $X$. Let $\mathbb{H} \subset \mathbb{C}$ denote the closed upper half-plane, and $\mathbb{D}^{+}:=\mathbb{D} \cap \mathbb{H}$. We now collect some lemmas that will be useful in the compactness arguments to come.

Lemma 3.8 (Hofer-Zehnder [17, Section 6.4, Lemma 5]) Let $(X, d)$ be a complete metric space and $f: X \rightarrow[0, \infty)$ a continuous function. Then given any $x_{0} \in X$ and $\epsilon_{0}>0$, there exist $x \in \overline{B_{2 \epsilon_{0}}\left(x_{0}\right)}$ and $\epsilon \in\left(0, \epsilon_{0}\right]$ such that

$$
f(x) \epsilon \geq f\left(x_{0}\right) \epsilon_{0} \quad \text { and } \quad f(y) \leq 2 f(x) \text { for all } y \in \overline{B_{\epsilon}(x)} .
$$

The next statement follows from the fact that when $\tilde{u}=(a, u): \dot{\Sigma} \rightarrow \mathbb{R} \times M$ is a finite energy surface, the map $a$ is both proper and subharmonic (cf [12]). Note however that in non-contact cases, there can generally exist nonconstant closed $\widetilde{J}$-holomorphic curves in $\mathbb{R} \times M$.

Lemma 3.9 Every finite energy surface with nonremovable punctures and no boundary has at least one positive puncture.

Lemma 3.10 Suppose $(\underset{\sim}{\Sigma}, j)$ is a simply connected Riemann surface and $\tilde{u}=$ $(a, u):(\Sigma, j) \rightarrow(\mathbb{R} \times M, \widetilde{J})$ is pseudoholomorphic with $E_{\omega}(\widetilde{u})=0$. Then $\tilde{u}$ has the form

$$
\tilde{u}(z)=(a(z), x(f(z)))
$$

where $x: \mathbb{R} \rightarrow M$ is an orbit of $X$ (not necessarily periodic) and $a+i f: \Sigma \rightarrow \mathbb{C}$ is a holomorphic function.

Proof The integrand of $E_{\omega}(\tilde{u})=\int_{\Sigma} u^{*} \omega$ is everywhere nonnegative, and vanishes at $z \in \Sigma$ if and only if the image of $d u(z)$ is contained in $\mathbb{R} X(u(z))$. By assumption this is true everywhere, thus $u(\Sigma)$ is contained in the image of some orbit $x: \mathbb{R} \rightarrow M$ of $X$. If $x$ is not periodic, then it's a diffeomorphism onto its image and can be inverted, allowing us to find a function $f: \Sigma \rightarrow \mathbb{R}$ such that $u=x \circ f$. If $x$ is periodic, then it can be viewed as a covering map onto its image, and the simple connectedness of $\Sigma$ means that $u$ can be lifted to the cover, producing again the map $f$. An easy computation now shows that $\tilde{u}=(a, u)$ is $\widetilde{J}$-holomorphic if and only if $a+i f$ satisfies the standard Cauchy-Riemann equations. 
Proposition 3.11 Suppose $\tilde{u}$ : $\Sigma \rightarrow \mathbb{R} \times M$ is $\widetilde{J}$-holomorphic with finite energy $E(\tilde{u})<\infty, E_{\omega}(\tilde{u})=0$ and $\tilde{u}(\partial \Sigma) \subset\{0\} \times L$.

(1) If $\Sigma$ is $S^{2}, \mathbb{D}, \mathbb{C}$ or $\mathbb{H}$, then $\tilde{u}$ is constant.

(2) If $\Sigma$ is $\mathbb{R} \times S^{1}$ or $(-\infty, 0] \times S^{1}$, there exists a number $Q \in \mathbb{R}$ and an orbit $x: \mathbb{R} \rightarrow M$ of $X$ such that up to $\mathbb{R}$-translation, $\tilde{u}(s, t)=(Q s, x(Q t))$.

Proof Applying Lemma 3.10, the function $a$ is harmonic, and constant on the boundary. For the compact cases $\Sigma=S^{2}$ and $\mathbb{D}$ this is enough: we conclude that $a$ is globally constant, and so therefore is $f$, its harmonic conjugate.

For $\Sigma=\mathbb{C}$, an argument from Hofer [10, Lemma 28] uses the finiteness of $E_{\lambda}(\widetilde{u})$ to show that $\Phi:=a+i f$ is constant. In brief, the energy can be rewritten as

$$
E_{\lambda}(\tilde{u})=\sup _{\varphi \in \mathcal{T}} \int_{\mathbb{C}} \tilde{u}^{*}(d \varphi \wedge \lambda)=\sup _{\varphi \in \mathcal{T}} \int_{\mathbb{C}} \Phi^{*} d(\varphi(s) d t),
$$

and a bubbling off argument shows that $\Phi$ must be constant if the latter is finite. We use this argument also for the case $\Sigma=\mathbb{H}$, after observing that the boundary condition implies $\Phi(\mathbb{R}) \subset i \mathbb{R}$, so $\Phi$ extends by the Schwartz reflection principle to an entire function on $\mathbb{C}$.

The cylinder cases also follow from arguments in [10]: a simple bubbling off argument using the finite energy shows that $|d \tilde{u}|$ is globally bounded, and we then lift the domain to $\mathbb{C}$ or $\mathbb{H}$ and apply Lemma 3.10. The holomorphic function $\Phi=a+i f$ need not be constant in this case but is affine due to the gradient bound, so periodicity in $t$ implies $\Phi(s, t)=Q s+c+i Q t$ for some constants $Q, c \in \mathbb{R}$, and $x$ is $|Q|$-periodic unless $Q=0$.

We will often use the fact that interior punctures of holomorphic curves with finite energy are either asymptotic to periodic orbits or are removable; the latter is the case whenever the image is contained within a compact subset of $\mathbb{R} \times M$. This follows from the standard theorem on removal of singularities (cf McDuff-Salamon [23]), together with the following observation: if $\varphi: \mathbb{R} \rightarrow(0, \epsilon)$ is a smooth increasing function and $\epsilon$ is sufficiently small, then

$$
d(\varphi \lambda)+\omega
$$

is a symplectic form on $\mathbb{R} \times M$, and any $\widetilde{J}$-holomorphic map $\tilde{u}$ with $E(\tilde{u})<\infty$ also has finite symplectic area with respect to this form.

We will need a corresponding statement for boundary punctures. Since our usual boundary condition on curves $\tilde{u}: \dot{\Sigma} \rightarrow \mathbb{R} \times M$ constrains $\tilde{u}(\partial \Sigma)$ to lie in a compact 
submanifold of $\mathbb{R} \times M$, one expects boundary punctures to removable. One only needs to show that such maps cannot become unbounded on the interior in a neighborhood of a puncture; we will show that this is always the case when the boundary condition has the form $\widetilde{L}=\{$ const $\} \times L .^{1}$

Theorem 3.12 (Removal of boundary singularities) Suppose $\tilde{u}=(a, u)$ : $\dot{\mathbb{D}}^{+}=$ $\mathbb{D}^{+} \backslash\{0\} \rightarrow \mathbb{R} \times M$ is a $\widetilde{J}$-holomorphic map with $E(\tilde{u})<\infty$ and $\tilde{u}\left(\dot{D}^{+} \cap \mathbb{R}\right) \subset\{0\} \times L$. Then $\tilde{u}$ extends to a $\widetilde{J}$-holomorphic half-disk $\mathbb{D}^{+} \rightarrow \mathbb{R} \times M$ with $\tilde{u}\left(\mathbb{D}^{+} \cap \mathbb{R}\right) \subset\{0\} \times L$.

Proof By the remarks above, the result will follow from the standard removal of singularities theorem after showing that $\widetilde{u}\left(\dot{\mathbb{D}}^{+}\right)$is contained in a compact subset of $\mathbb{R} \times M$. To see that this is the case, compose $\tilde{u}$ with the biholomorphic map $\psi:[0, \infty) \times[0,1] \rightarrow \dot{\mathbb{D}}^{+}:(s, t) \mapsto e^{-\pi(s+i t)}$ and consider the pseudoholomorphic half-strip

$$
\tilde{v}=(b, v)=\tilde{u} \circ \psi:[0, \infty) \times[0,1] \rightarrow \mathbb{R} \times M .
$$

We claim that $|d \widetilde{v}|$ is bounded on $[0, \infty) \times[0,1]$, where the norm is defined with respect to the Euclidean metric on $[0, \infty) \times[0,1] \subset \mathbb{C}$ and any fixed $\mathbb{R}$-invariant metric on $\mathbb{R} \times M$. It will follow from this that $\widetilde{v}$ (and hence $\tilde{u}$ ) is bounded, as $\widetilde{v}([0, \infty) \times\{0\}$ ) and $\tilde{v}([0, \infty) \times\{1\})$ are contained in the compact set $\{0\} \times L$.

If $|d \widetilde{v}|$ is not bounded, there is a sequence $z_{k}=s_{k}+i t_{k} \in[0, \infty) \times[0,1] \subset \mathbb{C}$ such that $R_{k}:=\left|d \widetilde{v}\left(z_{k}\right)\right| \rightarrow \infty$. We may assume $s_{k} \rightarrow \infty$. Choose a sequence of positive numbers $\epsilon_{k} \rightarrow 0$ such that $\epsilon_{k} R_{k} \rightarrow \infty$; by Lemma 3.8 we can assume without loss of generality that $|d \widetilde{v}(z)| \leq 2\left|d \widetilde{v}\left(z_{k}\right)\right|$ whenever $\left|z-z_{k}\right| \leq \epsilon_{k}$. We will define a sequence of rescaled maps which converge to either a plane or a half-plane, depending on whether and how fast $z_{k}$ approaches the boundary of $[0, \infty) \times[0,1]$. We consider three cases.

Case 1 Assume $t_{k} R_{k}$ and $\left(1-t_{k}\right) R_{k}$ are both unbounded then we can pass to a subsequence so that both approach $\infty$. Let

$$
r_{k}:=\min \left\{\epsilon_{k} R_{k}, t_{k} R_{k},\left(1-t_{k}\right) R_{k}\right\},
$$

so $r_{k} \rightarrow \infty$ and there are embeddings

$$
\psi_{k}: \mathbb{D}_{r_{k}} \hookrightarrow[0, \infty) \times[0,1]: z \mapsto z_{k}+\frac{z}{R_{k}} .
$$

Use these to define rescaled maps

$$
\widetilde{v}_{k}=\left(b \circ \psi_{k}-b\left(z_{k}\right), v \circ \psi_{k}\right): \mathbb{D}_{r_{k}} \rightarrow \mathbb{R} \times M,
$$

\footnotetext{
${ }^{1}$ As shown in [29], a somewhat more general statement is true, but it's not clear whether these arguments can be generalized to accommodate arbitrary graphs $\widetilde{L}=\{(g(x), x) \in \mathbb{R} \times L\}$.
} 
which satisfy a uniform $C^{1}$-bound and have a subsequence convergent in $C_{\text {loc }}^{\infty}$ to a non-constant $\widetilde{J}$-holomorphic plane $\widetilde{v}_{\infty}: \mathbb{C} \rightarrow \mathbb{R} \times M$. This map has finite energy $E\left(\widetilde{v}_{\infty}\right) \leq E(\widetilde{v})<\infty$, but also $E_{\omega}\left(\widetilde{v}_{\infty}\right)=0$, giving a contradiction to Proposition 3.11 .

Case 2 Assume $t_{k} R_{k}$ is bounded. This means $z_{k}$ is approaching the half-line $[0, \infty) \times$ $\{0\}$. Let

$$
\psi_{k}: \mathbb{D}_{\epsilon_{k} R_{k}}^{+} \hookrightarrow[0, \infty) \times[0,1]: z \mapsto s_{k}+\frac{z}{R_{k}},
$$

and define a sequence of rescaled maps $\tilde{v}_{k}: \mathbb{D}_{\epsilon_{k} R_{k}}^{+} \rightarrow \mathbb{R} \times M$ by $\tilde{v}_{k}=\tilde{v} \circ \psi_{k}$. These maps satisfy the boundary condition $\tilde{v}_{k}\left(\mathbb{D}_{\epsilon_{k} R_{k}} \cap \mathbb{R}\right) \subset\{0\} \times L$. Moreover, the points $\widetilde{v}_{k}(0)=\widetilde{v}\left(s_{k}\right)$ are contained in the compact set $\{0\} \times L$, and there is a uniform gradient bound $\left|d \widetilde{v}_{k}(z)\right| \leq 2$ for all $z \in \mathbb{D}_{\epsilon_{k} R_{k}}^{+}$. Thus a subsequence converges in $C_{\text {loc }}^{\infty}(\mathbb{H}, \mathbb{R} \times M)$ to a $\widetilde{J}$-holomorphic half-plane $\tilde{v}_{\infty}: \mathbb{H} \rightarrow \mathbb{R} \times M$ with $E\left(\widetilde{v}_{\infty}\right)<\infty$ and $E_{\omega}\left(\widetilde{v}_{\infty}\right)=0$. We claim however that $\widetilde{v}_{\infty}$ is not constant. Indeed, $\left|\psi_{k}^{-1}\left(z_{k}\right)\right|=R_{k}\left|z_{k}-s_{k}\right|=t_{k} R_{k}$ is bounded, thus passing to a subsequence, $\psi_{k}^{-1}\left(z_{k}\right) \rightarrow \zeta \in \mathbb{H}$, and $\left|d \tilde{v}_{\infty}(\zeta)\right|=\lim _{k}\left|d \widetilde{v}_{k}\left(\psi_{k}^{-1}\left(z_{k}\right)\right)\right|=1$. Thus the existence of $\tilde{v}_{\infty}$ again contradicts Proposition 3.11.

Case 3 Assume $\left(1-t_{k}\right) R_{k}$ is bounded. This is very similar to the previous case; this time $z_{k}$ is approaching the half-line $[0, \infty) \times\{1\}$, so we rescale using the embeddings

$$
\psi_{k}: \mathbb{D}_{\epsilon_{k} R_{k}}^{+} \hookrightarrow[0, \infty) \rightarrow[0,1]: z \mapsto\left(s_{k}+i\right)-\frac{z}{R_{k}} .
$$

Then by the same arguments used above, $\tilde{v}_{k}=\tilde{v} \circ \psi_{k}$ has a subsequence convergent to a non-constant finite energy half-plane $\widetilde{v}_{\infty}: \mathbb{H} \rightarrow \mathbb{R} \times M$ with boundary condition $\widetilde{v}_{\infty}(\mathbb{R}) \subset\{0\} \times L$ and $E_{\omega}\left(\widetilde{v}_{\infty}\right)=0$, giving another contradiction.

\subsection{Taming forms and energy bounds}

We now proceed toward the proofs of Theorem 3.4 and Theorem 3.5, so let $M, K$, $L_{j}, \mathcal{H}_{k}$ and $\tilde{u}_{k}$ be as defined in Section 3.1. Observe that since $X_{k}$ is always tangent to the tori $L_{j}$, the $2-$ forms $d \lambda_{k}$ and $\omega_{k}$ arising from $\mathcal{H}_{k}$ vanish on $L_{j}$.

Lemma 3.13 The taming forms $\omega_{k}$ for $k \leq \infty$ are exact on $M$.

Proof Applying a Mayer-Vietoris sequence to $S^{3}=M \cup N, H_{2}(M)$ is generated by the inclusions of the fundamental classes $\left[L_{j}\right] \in H_{2}\left(L_{j}\right)$ for $j=1, \ldots, m$. Then $\int_{L_{j}} \omega_{k}=0$ implies that $\omega_{k}$ vanishes on $H_{2}(M ; \mathbb{R})$, hence $\left[\omega_{k}\right]=0 \in H^{2}(M ; \mathbb{R})$. 
Denote by $E_{k}\left(\tilde{u}_{k}\right)$ the energy of $\tilde{u}_{k}$, computed with respect to $\omega_{k}$ and $\lambda_{k}$. Surfaces of the form $\{$ const $\} \times L_{j}$ are not only totally real in $(\mathbb{R} \times M, \widetilde{J})$ but also Lagrangian with respect to natural symplectic forms as in (10). This will permit a uniform energy bound for the sequence $\tilde{u}_{k}$.

Lemma 3.14 $\int_{\dot{\Sigma}} u_{k}^{*} \omega_{k}$ is uniformly bounded for all $k$.

Proof Since $\omega_{k}$ is exact and vanishes on each torus $L_{j}, \int_{\dot{\Sigma}} u_{k}^{*} \omega_{k}$ depends only on the asymptotic behavior and the homotopy class of $\left.u_{k}\right|_{\partial \Sigma}: \partial \Sigma \rightarrow \partial M$. Thus we can pick any smooth map $u: \dot{\Sigma} \rightarrow M$ with the proper behavior and write $\int_{\dot{\Sigma}} u_{k}^{*} \omega_{k}=$ $\int_{\dot{\Sigma}} u^{*} \omega_{k} \rightarrow \int_{\dot{\Sigma}} u^{*} \omega_{\infty}$.

Lemma 3.15 There exists a constant $C>0$ independent of $k$ such that $\left|u^{*} d \lambda_{k}\right| \leq$ $C \cdot u^{*} \omega_{k}$ for every $\widetilde{J}_{k}$-holomorphic curve $\tilde{u}=(a, u)$ in $\mathbb{R} \times M$.

Proof Let $C_{k}>0$ be the $C^{0}$-norm of the bilinear form

$$
\xi_{k} \times \xi_{k} \rightarrow \mathbb{R}:(v, w) \mapsto d \lambda_{k}\left(v, J_{k} w\right)
$$

on the bundle $\xi_{k} \rightarrow M$ with respect to the bundle metric $|v|_{k}^{2}:=\omega_{k}\left(v, J_{k} v\right)$; this is finite since $M$ is compact. Since $\omega_{k}$ and $\lambda_{k}$ each converge in $C^{\infty}$ as $k \rightarrow \infty$, the sequence $C_{k}$ is also bounded, $C_{k} \leq C$. Denote by $\pi_{k}: T M \rightarrow \xi_{k}$ the projection along $X_{k}$, and note that both $d \lambda_{k}$ and $\omega_{k}$ annihilate $X_{k}$. Then in any local holomorphic coordinate system $(s, t)$ on the domain,

$$
\begin{aligned}
\left|u^{*} d \lambda_{k}\left(\partial_{s}, \partial_{t}\right)\right| & =\left|d \lambda_{k}\left(\pi_{k} u_{s}, \pi_{k} u_{t}\right)\right|=\left|d \lambda_{k}\left(\pi_{k} u_{s}, J_{k} \pi_{k} u_{s}\right)\right| \\
& \leq C_{k}\left|\pi_{k} u_{s}\right|_{k}^{2} \leq C \cdot \omega_{k}\left(\pi_{k} u_{s}, J_{k} \pi_{k} u_{s}\right) \\
& =C \cdot \omega_{k}\left(\pi_{k} u_{s}, \pi_{k} u_{t}\right)=C \cdot u_{k}^{*} \omega_{k}\left(\partial_{s}, \partial_{t}\right) .
\end{aligned}
$$

This completes the proof.

Proposition 3.16 There exists a constant $C>0$ such that $E_{k}\left(\tilde{u}_{k}\right)<C$.

Proof Writing $E_{k}\left(\tilde{u}_{k}\right)=E_{\omega_{k}}\left(\tilde{u}_{k}\right)+E_{\lambda_{k}}\left(\tilde{u}_{k}\right)$, the first term is uniformly bounded due to Lemma 3.14. The second is $\sup _{\varphi \in \mathcal{T}} E_{\lambda_{k}}^{\varphi}\left(\tilde{u}_{k}\right)$, where

$$
E_{\lambda_{k}}^{\varphi}\left(\tilde{u}_{k}\right):=\int_{\dot{\Sigma}} \tilde{u}_{k}^{*}\left(d \varphi \wedge \lambda_{k}\right)
$$


Writing $d\left(\varphi \lambda_{k}\right)=\varphi d \lambda_{k}+d \varphi \wedge \lambda_{k}$, then applying Stokes' theorem, Lemma 3.15 and the fact that $a_{k}$ is locally constant at $\partial \Sigma$, we have

$$
\begin{aligned}
\left|E_{\lambda_{k}}^{\varphi}\left(\tilde{u}_{k}\right)\right| & \leq\left|\int_{\dot{\Sigma}} \tilde{u}_{k}^{*}\left(\varphi d \lambda_{k}\right)\right|+\left|\int_{\dot{\Sigma}} \tilde{u}_{k}^{*} d\left(\varphi \lambda_{k}\right)\right| \\
& \leq \int_{\dot{\Sigma}}\left|\varphi \circ a_{k}\right| \cdot\left|u_{k}^{*} d \lambda_{k}\right|+\int_{P_{\infty}} \lambda_{k}+\left|\int_{\partial \Sigma} \tilde{u}_{k}^{*}\left(\varphi \lambda_{k}\right)\right| \\
& \leq C \int_{\dot{\Sigma}} u_{k}^{*} \omega_{k}+T_{k}+\left|\int_{\partial \Sigma} u_{k}^{*} \lambda_{k}\right|
\end{aligned}
$$

where $T_{k}$ is the period of $P_{\infty}$ as an orbit of $X_{k}$. This is clearly bounded as $k \rightarrow \infty$, and so is the first term, by Lemma 3.14 ; it remains only to bound $\int_{\partial \Sigma} u_{k}^{*} \lambda_{k}$. Here we use the fact that $d \lambda_{k}\left(X_{k}, \cdot\right) \equiv 0$, hence $d \lambda_{k}$ annihilates each $L_{j}$, and this integral therefore only depends on $\lambda_{k}$ and $\left[\left.u_{k}\right|_{\partial \Sigma}\right]=\ell \in H_{1}\left(L_{1} \cup \cdots \cup L_{m}\right)$. In particular, it approaches $\int_{\ell} \lambda_{\infty}$ as $k \rightarrow \infty$.

\subsection{Bubbling}

In this section we establish uniform bounds on the first derivatives of the maps $\tilde{u}_{k}$. In the non-stable $(m<2)$ case, the arguments of this section suffice to prove $C_{\mathrm{loc}}^{\infty}-$ convergence. For $m \geq 2$, we also need to ensure that the sequence of conformal structures induced by $j_{k}$ is compact; this issue will be dealt with in Section 3.6. The fundamental argument is that any gradient blow up causes the bubbling off of a holomorphic plane or disk, which for topological reasons cannot exist.

We focus first on the stable case, thus assume $m \geq 2, \chi(\dot{\Sigma})<0$. Then each of the stable Riemann surfaces $\left(\Sigma, j_{k},\{\infty\}\right)$ determines a Poincaré metric $h_{k}$, which is the restriction of a complete metric $h_{k}^{D}$ of constant curvature -1 on the Riemann surface

$$
\left(\dot{\Sigma}^{D}, j_{k}^{D}\right)
$$

obtained by doubling $\left(\dot{\Sigma}, j_{k}\right)$ along the boundary. Denote the injectivity radius of $h_{k}^{D}$ at any point $z \in \dot{\Sigma}$ by $\operatorname{injrad}_{k}(z)$.

Fix any metric on $M$ and extend it to an $\mathbb{R}$-invariant metric on $\mathbb{R} \times M$ in the natural way. In the following, we will always use the Euclidean metric on subsets of $\mathbb{C}$ or $\mathbb{R} \times S^{1}$, and one of the Poincaré metrics $h_{k}$ on $\dot{\Sigma}$, with dependence on $k$ reflected in the notation. So for instance, given $\tilde{u}: \dot{\Sigma} \rightarrow \mathbb{R} \times M,|d \tilde{u}(z)|_{k}$ is the norm of the linear map $d \tilde{u}(z): T_{z} \dot{\Sigma} \rightarrow T_{\widetilde{u}(z)}(\mathbb{R} \times M)$ with respect to $h_{k}$ and the fixed metric on $\mathbb{R} \times M$. For $\varphi: \mathbb{D} \rightarrow \dot{\Sigma}$, define $|d \varphi(z)|_{k}$ with respect to the Euclidean metric on the domain and $h_{k}$ on the target. 
The following technical lemma provides good coordinates in a neighborhood of any point $z_{0} \in \dot{\Sigma}$. They are constructed by lifting $\dot{\Sigma}$ to the hyperbolic open disk so that 0 covers $z_{0}$, then projecting the embedding $\mathbb{D} \hookrightarrow$ int $\mathbb{D}: z \mapsto r z$ for sufficiently small $r>0$ down to $\dot{\Sigma}$. It follows from the hyperbolic geometry of the disk that the resulting embedding has the desired properties; see [29] for details. Denote by $\mathbb{D}_{\rho}$ the standard closed disk of radius $\rho>0$ in $\mathbb{C}$.

Lemma 3.17 Let $(\dot{\Sigma}, j)$ be a stable punctured Riemann surface without boundary, with Poincaré metric $h$, whose injectivity radius at $z \in \dot{\Sigma}$ we denote by $\operatorname{injrad}(z)$. There are positive constants $c_{i}$ and $C_{i}$ depending only on the topological type of $\dot{\Sigma}$ (ie not on $j$ ), such that the following is true: for any $z_{0} \in \dot{\Sigma}$ and any geodesic $\gamma$ passing through $z_{0}$, there is a holomorphic embedding $\varphi: \mathbb{D} \hookrightarrow \dot{\Sigma}$ such that $\varphi(0)=z_{0}, \varphi$ maps $\mathbb{R} \cap \mathbb{D}$ to $\gamma$ preserving orientation, and

$$
c_{1} \cdot \operatorname{injrad}\left(z_{0}\right) \leq|d \varphi(z)|_{h} \leq C_{1} \cdot \operatorname{injrad}\left(z_{0}\right) \quad \text { for all } z \in \mathbb{D} .
$$

For any $\rho \in[0,1]$, the image $\varphi\left(\mathbb{D}_{\rho}\right)$ is then a closed ball of radius $d(\rho)$ in $(\dot{\Sigma}, h)$, where

$$
c_{2} \rho \cdot \operatorname{injrad}\left(z_{0}\right) \leq d(\rho) \leq C_{2} \rho \cdot \operatorname{injrad}\left(z_{0}\right),
$$

and the injectivity radius at any point $\varphi(w)$ for $w \in \mathbb{D}$ with $|w|=\rho$ can be estimated by

$$
\left(c_{3}-c_{4} \rho\right) \cdot \operatorname{injrad}\left(z_{0}\right) \leq \operatorname{injrad}(\varphi(w)) \leq\left(1+C_{3} \rho\right) \cdot \operatorname{injrad}\left(z_{0}\right) .
$$

Remark 3.18 Lemma 3.17 extends to surfaces $\dot{\Sigma}$ with nonempty boundary as follows: for any $z_{0} \in \partial \Sigma$, the component $\gamma \subset \partial \Sigma$ containing $z_{0}$ is a closed geodesic in the doubled surface $\left(\dot{\Sigma}^{D}, h^{D}\right)$. Thus the lemma gives an embedding $\varphi: \mathbb{D}^{+} \rightarrow \dot{\Sigma}$ of the upper half disk, sending 0 to $z_{0}$ and $\mathbb{R} \cap \mathbb{D}^{+}$into $\partial \Sigma$.

The first main objective in this section is the following result, which says in effect that there is no bubbling off in the sequence $\tilde{u}_{k}$.

Proposition 3.19 If $\chi(\dot{\Sigma})<0$, then there is a constant $C>0$ such that

$$
\left|d \tilde{u}_{k}(z)\right|_{k} \leq \frac{C}{\operatorname{injrad}_{k}(z)}
$$

for all $z \in \dot{\Sigma}$ and all $k$. 
Proof Assume there exists a sequence $z_{k} \in \dot{\Sigma}$ such that $\operatorname{injrad}_{k}\left(z_{k}\right) \cdot\left|d \widetilde{u}\left(z_{k}\right)\right|_{k} \rightarrow \infty$. Using Lemma 3.17, choose a sequence of holomorphic embeddings

$$
\varphi_{k}: \mathbb{D} \hookrightarrow \dot{\Sigma}^{D}
$$

such that $\left|d \varphi_{k}\right|_{k}$, the radii of the disks $\varphi_{k}(\mathbb{D})$ and the injectivity radius satisfy the bounds specified in the lemma. Let

$$
\rho_{k}=\min \left\{|\zeta| \mid \zeta \in \varphi_{k}^{-1}(\partial \Sigma)\right\},
$$

or $\rho_{k}=\infty$ if $\varphi_{k}(\mathbb{D}) \cap \partial \Sigma=\varnothing$. The sequence $\rho_{k}$ determines whether or not we can restrict the embeddings $\varphi_{k}$ in a uniform way so that their images are in $\dot{\Sigma}$.

Case 1 Assume there is a number $\rho \in(0,1]$ and a subsequence for which $\rho_{k} \geq \rho$. Then the restrictions of $\varphi_{k}$ to $\mathbb{D}_{\rho}$ are embeddings into $\dot{\Sigma}$, and we can define a sequence of pseudoholomorphic disks

$$
\tilde{v}_{k}=\left(b_{k}, v_{k}\right)=\tilde{u}_{k} \circ \varphi_{k}: \mathbb{D}_{\rho} \rightarrow \mathbb{R} \times S^{3},
$$

which satisfy a uniform energy bound

$$
E_{k}\left(\widetilde{v}_{k}\right) \leq E_{k}\left(\widetilde{u}_{k}\right) \leq C .
$$

Denoting the Euclidean metric on $\mathbb{D}$ by $\eta$, the fact that $\varphi_{k}:\left(\mathbb{D}_{\rho}, \eta\right) \rightarrow\left(\dot{\Sigma}, h_{k}\right)$ is conformal implies that the norms of $d \varphi_{k}(z)$ and its inverse are reciprocals. Then a simple computation shows

$$
\left|d \widetilde{v}_{k}(0)\right|=\left|d \tilde{u}_{k}\left(z_{k}\right)\right|_{k} \cdot\left|d \varphi_{k}(0)\right|_{k} \geq c_{1}\left|d \tilde{u}_{k}\left(z_{k}\right)\right|_{k} \cdot \operatorname{injrad}_{k}\left(z_{k}\right) \rightarrow \infty .
$$

By Lemma 3.8, we can choose a sequence $\zeta_{k} \in \mathbb{D}_{\rho}$ and positive numbers $\epsilon_{k} \rightarrow 0$ such that $R_{k}:=\left|d \widetilde{v}\left(\zeta_{k}\right)\right| \rightarrow \infty, \epsilon_{k} R_{k} \rightarrow \infty$ and $|d \widetilde{v}(\zeta)| \leq 2 R_{k}$ for all $\zeta \in \mathbb{D}_{\rho}$ with $\left|\zeta-\zeta_{k}\right| \leq \epsilon_{k}$. Assume without loss of generality that $\overline{B_{\epsilon_{k}}\left(\zeta_{k}\right)} \subset \mathbb{D}_{\rho}$ and define

$$
\psi_{k}: \mathbb{D}_{\epsilon_{k} R_{k}} \rightarrow \overline{B_{\epsilon_{k}}\left(\zeta_{k}\right)}: \zeta \rightarrow \zeta_{k}+\frac{\zeta}{R_{k}}
$$

Then we can define a rescaled sequence of $\widetilde{J}_{k}$-holomorphic maps

$$
\tilde{w}_{k}=\left(\beta_{k}, w_{k}\right): \mathbb{D}_{\epsilon_{k} R_{k}} \rightarrow \mathbb{R} \times S^{3}
$$

by

$$
\left(\beta_{k}(\zeta), w_{k}(\zeta)\right)=\left(b_{k} \circ \psi_{k}(\zeta)-b_{k}\left(\zeta_{k}\right), v_{k} \circ \psi_{k}(\zeta)\right)
$$

These satisfy the uniform gradient bound $\left|d \widetilde{w}_{k}(\zeta)\right| \leq 2$, and they all map 0 into a compact subset of $\mathbb{R} \times S^{3}$, thus a subsequence converges in $C_{\text {loc }}^{\infty}$ to a $\widetilde{J}_{\infty}$-holomorphic plane

$$
\widetilde{w}_{\infty}=\left(\beta_{\infty}, w_{\infty}\right): \mathbb{C} \rightarrow \mathbb{R} \times S^{3} .
$$


The bound on $E_{k}\left(\widetilde{v}_{k}\right)$ gives also a bound on $E_{k}\left(\widetilde{w}_{k}\right)$ and thus implies $E_{\infty}\left(\widetilde{w}_{\infty}\right)<\infty$, so $\tilde{w}_{\infty}$ is a non-constant finite energy plane.

If the puncture at $\infty$ is removable, $\tilde{w}_{\infty}$ extends to a nonconstant $\widetilde{J}_{\infty}$-holomorphic sphere in $\mathbb{R} \times M$. Recall now that $\xi_{\infty}$ admits a global trivialization over $M$, thus $c_{1}\left(w_{\infty}^{*} \xi_{\infty}\right)=0$ and we have

$$
\operatorname{ind}\left(\widetilde{w}_{\infty}\right)=\mu\left(\widetilde{w}_{\infty}\right)-\chi\left(S^{2}\right)=2 c_{1}\left(w_{\infty}^{*} \xi_{\infty}\right)-2=-2 .
$$

Since $\widetilde{w}_{\infty}$ is nonconstant, it cannot have $E_{\omega_{\infty}}\left(\widetilde{w}_{\infty}\right)=0$, by Proposition 3.11. Thus $E_{\omega_{\infty}}\left(\widetilde{w}_{\infty}\right)>0$ and Theorem 2.4 gives a contradiction, in the form

$$
0 \leq 2 \operatorname{wind}_{\pi}\left(\tilde{w}_{\infty}\right) \leq \operatorname{ind}(\widetilde{w})-2=-4 .
$$

It remains to find a contradiction in the case of a non-removable puncture at $\infty$ : then $\widetilde{w}_{\infty}$ is asymptotic to some periodic orbit $P$ of $X_{\infty}$. We now use a topological argument to show that this is impossible.

If $P$ is geometrically distinct from $P_{\infty}$, then $\operatorname{lk}\left(P, P_{\infty}\right) \neq 0$ by assumption. For some large radius $R$, the image $w_{\infty}\left(\partial \mathbb{D}_{R}\right)$ is uniformly close to $P$, and we may assume the same is true of $P^{\prime}:=w_{k}\left(\partial \mathbb{D}_{R}\right)$ for sufficiently large $k$, thus $\operatorname{lk}\left(P^{\prime}, P_{\infty}\right) \neq 0$. But since $w_{k}$ is a reparametrization of $u_{k}: \dot{\Sigma} \rightarrow S^{3}$ over some disk, this means there is a disk $\mathcal{D} \subset \dot{\Sigma}$ such that $P^{\prime}=u_{k}(\partial \mathcal{D})$. The linking condition then implies that $u_{k}(\mathcal{D})$ intersects $P_{\infty}$, contradicting the result of Corollary 3.3.

Suppose now that $P$ is identical to $P_{\infty}$ or some cover thereof. For any component $K_{j} \subset K$, observe that $u_{k}(\dot{\Sigma})$ never intersects $K_{j}$. Then repeating the argument above, we find a disk $\mathcal{D} \subset \dot{\Sigma}$ such that for sufficiently large $k, u_{k}(\partial \mathcal{D})$ is a knot uniformly close to $P_{\infty}$. This implies $\operatorname{lk}\left(P_{\infty}, K_{j}\right)=0$, another contradiction, thus proving that the plane $\tilde{w}_{\infty}$ cannot exist.

Case 2 Assume $\rho_{k} \rightarrow 0$. Here we will find that either a plane or a disk bubbles off, depending on how fast $\rho_{k}$ approaches 0 . Choose a sequence $\zeta_{k}^{\prime} \in \mathbb{D}$ such that $\zeta_{k}:=\varphi_{k}\left(\zeta_{k}^{\prime}\right) \in \partial \Sigma$ and $\left|\zeta_{k}^{\prime}\right|=\rho_{k}$. By Remark 3.18, we can find a sequence of holomorphic embeddings

$$
\varphi_{k}^{+}: \mathbb{D}^{+} \hookrightarrow \dot{\Sigma}
$$

that map 0 to $\zeta_{k}$ and $\mathbb{D}^{+} \cap \mathbb{R}$ into $\partial \Sigma$, and satisfy the bounds specified in Lemma 3.17. We claim there is a sequence of radii $r_{k} \rightarrow 0$ such that $z_{k} \in \varphi_{k}^{+}\left(\mathbb{D}_{r_{k}}^{+}\right)$. Indeed, from Lemma 3.17, we know that $\varphi^{+}\left(\mathbb{D}_{r_{k}}^{+}\right)$contains all points $\zeta \in \dot{\Sigma}$ with $\operatorname{dist}_{h_{k}}\left(\zeta, \zeta_{k}\right) \leq d_{k}$, where

$$
d_{k} \geq c_{2} r_{k} \cdot \operatorname{injrad}_{k}\left(\zeta_{k}\right)
$$


We have also the estimates

$$
\begin{aligned}
\operatorname{dist}_{h_{k}}\left(z_{k}, \zeta_{k}\right) & \leq C_{2} \rho_{k} \cdot \operatorname{injrad}_{k}\left(z_{k}\right), \\
\quad \operatorname{injrad}_{k}\left(\zeta_{k}\right) & \geq\left(c_{3}-c_{4} \rho_{k}\right) \cdot \operatorname{injrad}_{k}\left(z_{k}\right)
\end{aligned}
$$

Then when $\rho_{k}$ is sufficiently small we can set

$$
r_{k}=\frac{2 C_{2}}{c_{2}\left(c_{3}-c_{4} \rho_{k}\right)} \rho_{k}
$$

and compute,

$$
\operatorname{dist}_{h_{k}}\left(\zeta_{k}, z_{k}\right) \leq \frac{C_{2}}{c_{3}-c_{4} \rho_{k}} \rho_{k} \cdot \operatorname{injrad}_{k}\left(\zeta_{k}\right)=\frac{1}{2} c_{2} r_{k} \operatorname{injrad}_{k}\left(\zeta_{k}\right)<d_{k} .
$$

We can thus choose a sequence $z_{k}^{\prime} \in \mathbb{D}^{+}$with $z_{k}^{\prime} \rightarrow 0$ and $\varphi_{k}^{+}\left(z_{k}^{\prime}\right)=z_{k}$. Defining a sequence of $\widetilde{J}_{k}$-holomorphic half-disks

$$
\tilde{v}_{k}=\tilde{u}_{k} \circ \varphi^{+}: \mathbb{D}^{+} \rightarrow \mathbb{R} \times S^{3},
$$

we have

$$
\begin{aligned}
R_{k}:=\left|d \widetilde{v}_{k}\left(z_{k}^{\prime}\right)\right| & =\left|d \widetilde{u}_{k}\left(z_{k}\right)\right|_{k} \cdot\left|d \varphi^{+}\left(z_{k}^{\prime}\right)\right|_{k} \geq C\left|d \tilde{u}_{k}\left(z_{k}\right)\right|_{k} \cdot \operatorname{injrad}_{k}\left(\zeta_{k}\right) \\
& \geq C\left(c_{3}-c_{4} \rho_{k}\right)\left|d \tilde{u}_{k}\left(z_{k}\right)\right|_{k} \cdot \operatorname{injrad}_{k}\left(z_{k}\right) \rightarrow \infty .
\end{aligned}
$$

Using Lemma 3.8, we may assume there is a sequence of positive numbers $\epsilon_{k} \rightarrow 0$ such that $\epsilon_{k} R_{k} \rightarrow \infty$ and $\left|d \widetilde{v}_{k}(z)\right| \leq 2 R_{k}$ for all $z \in \mathbb{D}^{+}$with $\left|z-z_{k}^{\prime}\right| \leq \epsilon_{k}$. Writing $z_{k}^{\prime}=s_{k}+i t_{k}$, there are two possibilities.

Case 2a Assume $t_{k} R_{k}$ is unbounded. Passing to a subsequence, we may assume $t_{k} R_{k} \rightarrow \infty$, thus $r_{k}^{\prime}:=\min \left\{\epsilon_{k} R_{k}, t_{k} R_{k}\right\} \rightarrow \infty$. Then for sufficiently large $k$ we can define embeddings $\psi_{k}: \mathbb{D}_{r_{k}^{\prime}} \hookrightarrow \mathbb{D}^{+}$by

$$
\psi_{k}(z)=z_{k}+\frac{z}{R_{k}}
$$

Arguing as in case 1, there is now a sequence of rescaled maps

$$
\widetilde{w}_{k}=\left(\beta_{k}, w_{k}\right)=\widetilde{v}_{k} \circ \psi_{k}: \mathbb{D}_{r_{k}^{\prime}} \rightarrow \mathbb{R} \times S^{3}
$$

and constants $c_{k} \in \mathbb{R}$ such that a subsequence of $\left(\beta_{k}+c_{k}, w_{k}\right)$ converges in $C_{\text {loc }}^{\infty}$ to a nonconstant $\widetilde{J}_{\infty}$-holomorphic finite energy plane $\widetilde{w}_{\infty}=\left(\beta_{\infty}, w_{\infty}\right): \mathbb{C} \rightarrow \mathbb{R} \times S^{3}$, giving the same contradiction as before. 
Case 2b Assume $t_{k} R_{k}$ is bounded. Now define $\psi_{k}: \mathbb{D}_{\epsilon_{k} R_{k}}^{+} \hookrightarrow \mathbb{D}^{+}$by

$$
\psi_{k}(z)=s_{k}+\frac{z}{R_{k}}
$$

and let

$$
\tilde{w}_{k}=\left(\beta_{k}, w_{k}\right)=\tilde{v}_{k} \circ \psi_{k}: \mathbb{D}_{\epsilon_{k} R_{k}}^{+} \rightarrow \mathbb{R} \times S^{3} .
$$

Then $\left|d \widetilde{w}_{k}\right|$ is uniformly bounded. Passing to a subsequence, $\left(\beta_{k}-\beta_{k}(0), w_{k}\right)$ converges in $C_{\text {loc }}^{\infty}$ to a $\widetilde{J}_{\infty}$-holomorphic half-plane

$$
\widetilde{w}_{\infty}=\left(\beta_{\infty}, w_{\infty}\right): \mathbb{H} \rightarrow \mathbb{R} \times S^{3},
$$

satisfying $E_{\infty}\left(\widetilde{w}_{\infty}\right)<\infty$ and the boundary condition $\widetilde{w}_{\infty}(\mathbb{R}) \subset\{0\} \times L_{j}$ for some $j \in\{1, \ldots, m\}$. It is not constant, since $\left|d \widetilde{w}_{k}\left(i t_{k} R_{k}\right)\right|=\frac{1}{R_{k}}\left|d \widetilde{v}_{k}\left(s_{k}+i t_{k}\right)\right|=1$ and a subsequence of $i t_{k} R_{k}$ converges in $\mathbb{D}^{+}$. Now identifying $\mathbb{H}$ conformally with $\mathbb{D} \backslash\{1\}$, we can regard $\widetilde{w}_{\infty}$ as a holomorphic disk with a puncture on the boundary, and Theorem 3.12 tells us that the puncture is removable. Thus extending over the puncture defines a $\widetilde{J}_{\infty}$-holomorphic disk

$$
\tilde{w}=(\beta, w): \mathbb{D} \rightarrow \mathbb{R} \times S^{3}
$$

with $w(\partial \mathbb{D}) \subset L_{j}$. By topological considerations, we can severely restrict the homotopy class of the loop $\gamma=\left.w\right|_{\partial \mathbb{D}}: \partial \mathbb{D} \rightarrow L_{j}$. Indeed, choose a radius $r$ slightly less than 1 so that $\left.w\right|_{\partial \mathbb{D}_{r}}: \partial \mathbb{D}_{r} \rightarrow S^{3}$ is uniformly close to $\gamma$. Returning to the half-plane $\mathbb{H}$, there is then a large simply connected region $\Omega \subset \mathbb{H}$ with smooth boundary such that for large $k,\left.w_{k}\right|_{\partial \Omega}: \partial \Omega \rightarrow S^{3}$ is also uniformly close to $\gamma$. Undoing the reparametrization one step further, there is then an embedded disk $\mathcal{D} \subset \dot{\Sigma}$ such that for some large $k$,

$$
\left.u_{k}\right|_{\partial \mathcal{D}}: \partial \mathcal{D} \rightarrow S^{3}
$$

is uniformly close to $\gamma$. Since $u_{k}$ does not intersect either $P_{\infty}$ or any of the knots $K_{j} \subset K$, this implies

$$
\operatorname{lk}\left(\gamma, P_{\infty}\right)=\operatorname{lk}\left(\gamma, K_{1}\right)=\cdots=\operatorname{lk}\left(\gamma, K_{m}\right)=0 .
$$

This is only possible if $\gamma$ is contractible on $L_{j}$. But this implies that the Maslov index $\mu(\widetilde{w})$ is zero. In this case $\operatorname{ind}(\tilde{w})=-\chi(\mathbb{D})+1=0$, and Theorem 2.4 gives

$$
0 \leq 2 \operatorname{wind}_{\pi}(\widetilde{w}) \leq \operatorname{ind}(\widetilde{w})-2=-2,
$$

unless $E_{\omega_{\infty}}(\widetilde{w})=0$. The latter is also impossible by Proposition 3.11, since $\widetilde{w}$ is not constant.

Having proved the gradient bound when $m \geq 2$, we now apply similar arguments for $m \leq 1$ and finish the proof of $C_{\mathrm{loc}}^{\infty}$-covergence for this case. 
Proposition 3.20 The statement about $C_{\mathrm{loc}}^{\infty}$-convergence in Theorem 3.4 holds if $\chi(\dot{\Sigma}) \geq 0$.

Proof This includes two cases: $\dot{\Sigma}$ is diffeomorphic to either a plane or a singly punctured disk. In both cases the space of conformal structures on the domain is trivial, so we can assume $\left(\dot{\Sigma}, j_{k}\right)$ is either $(\mathbb{C}, i)$ or $(\mathbb{C} \backslash \mathcal{D}, i)$ for all $k$, where $\mathcal{D}=$ int $\mathbb{D}$. We then have a sequence of maps $\tilde{u}_{k}=\left(a_{k}, u_{k}\right): \dot{\Sigma} \rightarrow \mathbb{R} \times S^{3}$ satisfying $T \tilde{u}_{k} \circ i=\widetilde{J}_{k} \circ T \tilde{u}$, all positively asymptotic at $\infty \in \Sigma$ to the simply covered orbit $P_{\infty}$.

We address first the case $\dot{\Sigma}=\mathbb{C}$; then there is no boundary condition and $M=$ $S^{3}$. By the same rescaling argument as in Proposition 3.19, we may assume after reparametrization that $\left|d \tilde{u}_{k}(z)\right|$ satisfies a global uniform bound and that $\left|d \tilde{u}_{k}(0)\right|$ is bounded away from zero. Then a subsequence of $\left(a_{k}-a_{k}(0), u_{k}\right)$ converges in $C_{\text {loc }}^{\infty}$ to a nonconstant $\widetilde{J}_{\infty}$-holomorphic finite energy plane $\tilde{u}_{\infty}=\left(a_{\infty}, u_{\infty}\right): \mathbb{C} \rightarrow \mathbb{R} \times S^{3}$. If the puncture is removable, then just as in Proposition 3.19, $\tilde{u}$ extends to a nonconstant sphere of index -2 , which violates Theorem 2.4 . Thus $\tilde{u}_{\infty}$ is positively asymptotic to a periodic orbit $P$, which we claim must be $P_{\infty}$. Indeed, if $P$ and $P_{\infty}$ are geometrically distinct, then the same linking argument implies $\operatorname{lk}\left(P, P_{\infty}\right)=0$, a contradiction. Suppose now that $P$ is an $n$-fold cover of $P_{\infty}$ for some integer $n \geq 1$. Writing $\omega_{k}=d \alpha_{k}$ by Lemma 3.13 and fixing any smooth map $u$ : $\mathbb{C} \rightarrow S^{3}$ that approaches $P_{\infty}$ asymptotically,

$$
E_{\omega_{k}}\left(\tilde{u}_{k}\right)=\int_{\mathbb{C}} u^{*} \omega_{k}=\int_{P_{\infty}} \alpha_{k} \rightarrow E_{\infty}:=\int_{\mathbb{C}} u^{*} \omega_{\infty}=\int_{P_{\infty}} \alpha_{\infty}
$$

But then

$$
n E_{\infty}=\int_{P} \alpha_{\infty}=\int_{\mathbb{C}} u_{\infty}^{*} \omega_{\infty} \leq \lim \int_{\mathbb{C}} u_{k}^{*} \omega_{k}=E_{\infty} .
$$

The left hand side equals $E_{\infty}\left(\tilde{u}_{\infty}\right)$ and must therefore be positive, so we conclude $n=1$.

Next suppose $\dot{\Sigma}=\mathbb{C} \backslash \mathcal{D}$. We claim that $\left|d \tilde{u}_{k}\right|$ is uniformly bounded. If not, then as in Proposition 3.19, we can define rescaled maps $\widetilde{v}_{k}$ on an increasing sequence of either disks or half-disks, depending on whether and how fast $z_{k}$ approaches the boundary. These then have a subsequence convergent to a non-constant finite energy plane or half-plane $\tilde{v}_{\infty}$. The usual arguments show that if $\widetilde{v}_{\infty}$ is a plane, it cannot be extended to a sphere, and the linking conditions on its asymptotic orbit force it to intersect either $P_{\infty}$ or $K$, neither of which is allowed. For the half-plane case, $\widetilde{v}$ extends to a non-constant pseudoholomorphic disk, and the same argument as before shows that $\widetilde{v}(\partial \mathbb{D})$ is contractible on $L$, thus its Maslov index is 0 , and it must therefore have vanishing $\omega_{\infty}$-energy, another contradiction. 
Given the uniform bound, there are constants $c_{k} \in \mathbb{R}$ and a subsequence of $\left(a_{k}+c_{k}, u_{k}\right)$ which converges in $C_{\text {loc }}^{\infty}$ to a $\widetilde{J}_{\infty}$-holomorphic finite energy map $\tilde{u}_{\infty}: \dot{\Sigma} \rightarrow \mathbb{R} \times M$, with the boundary condition $\tilde{u}_{\infty}(\partial \Sigma) \subset\{0\} \times L$. Then it remains to prove that $\tilde{u}_{\infty}$ has a positive puncture at $\infty$, asymptotic to $P_{\infty}$ with covering number 1 . If the puncture is removable, we obtain a holomorphic disk

$$
\mathbb{D} \rightarrow \mathbb{R} \times S^{3}: z \mapsto \tilde{u}_{\infty}(1 / z)
$$

mapping $\partial \mathbb{D}$ to a meridian on $L$, thus $u_{\infty}$ must intersect $K$. But then $u_{k}$ for large $k$ would have to intersect the interior of $N$, giving a contradiction. Now suppose $\tilde{u}_{\infty}$ is asymptotic to a periodic orbit $P$ at $\infty$. If we extend $\tilde{u}_{\infty}$ to a smooth map over $\mathbb{C}$, taking $\mathcal{D}$ into the solid torus $N$, then the same argument as in the plane case shows that $P$ cannot be geometrically distinct from $P_{\infty}$. Therefore $P$ is an $n$-fold cover of $P_{\infty}$ with $n \neq 0$. (Here we adopt the convention of setting $n$ to negative the covering number if the puncture is negative; this possibility is not excluded automatically when $\partial \Sigma \neq \varnothing$.) Now observe $u_{k}(\dot{\Sigma}) \cap K=\varnothing$ for all $k$, so if $k$ is sufficiently large, a small perturbation of $u_{k}$ realizes a homology $\partial\left[u_{k}\right]=n\left[P_{\infty}\right]+[\gamma]$ in $S^{3} \backslash K$, where $\gamma$ is a negatively oriented meridian on $L$. Consequently

$$
n \cdot \operatorname{lk}\left(P_{\infty}, K\right)=-\operatorname{lk}(\gamma, K)=1,
$$

and since $\operatorname{lk}\left(P_{\infty}, K\right)>0$ by assumption, $n$ can only be 1 .

\subsection{Convergence of conformal structures}

We now show that in the case $\chi(\dot{\Sigma})<0$, the induced sequence of conformal structures is compact.

Proposition 3.21 Given the hypotheses of Theorem 3.4, there is a smooth complex structure $j_{\infty}$ on $\Sigma$ and a sequence of diffeomorphisms $\varphi_{k}: \Sigma \rightarrow \Sigma$ fixing $\infty$ and preserving each component of $\partial \Sigma$, such that a subsequence of $\varphi_{k}^{*} j_{k}$ converges to $j_{\infty}$ in the $C^{\infty}$-topology.

There are also constants $c_{k} \in \mathbb{R}$ such that a subsequence of $\left(a_{k}+c_{k}, u_{k}\right) \circ \varphi_{k}$ converges in $C_{\text {loc }}^{\infty}(\dot{\Sigma}, \mathbb{R} \times M)$ to a map $\tilde{u}_{\infty} \in \mathcal{M}_{\mathcal{H}_{\infty}, \Lambda}$, which is positively asymptotic to $P_{\infty}$ at the puncture.

Proof A subsequence of $\left(\Sigma, j_{k},\{\infty\}\right)$ converges to a stable nodal surface $\mathbf{S}=$ $(S, j,\{p\}, \Delta, N)$. A choice of decoration $r$ defines the compact connected surface $\overline{\mathbf{S}}_{r}$, with a singular conformal structure $j_{\mathbf{S}}$ and singular Poincaré metric $h_{\mathbf{S}}$, both of 
which degenerate on a finite set of circles and $\operatorname{arcs} \Theta_{\Delta, N} \subset \overline{\mathbf{S}}_{r}$. Then convergence means there is a sequence of diffeomorphisms

$$
\varphi_{k}: \overline{\mathbf{S}}_{r} \rightarrow \Sigma
$$

such that:

(1) $\varphi_{k}(p)=\infty$,

(2) $\varphi_{k}^{*} j_{k} \rightarrow j_{\mathbf{S}}$ in $C_{\mathrm{loc}}^{\infty}\left(\overline{\mathbf{S}}_{r} \backslash \Theta_{\Delta, N}\right)$,

(3) all circles in $\varphi_{k}\left(\Theta_{\Delta, N}\right)$ are closed geodesics in $\left(\dot{\Sigma}, h_{k}\right)$, and all $\operatorname{arcs}$ in $\varphi_{k}\left(\Theta_{\Delta, N}\right)$ are geodesic arcs in $\left(\dot{\Sigma}, h_{k}\right)$ that intersect $\partial \Sigma$ transversely.

We can assume without loss of generality that the diffeomorphisms $\varphi_{k}$ map a given component of $\partial\left(\overline{\mathbf{S}}_{r}\right)$ always to the same component of $\partial \Sigma$, ie $\varphi_{k} \circ \varphi_{j}^{-1}$ always maps each connected component $\gamma_{j} \subset \partial \Sigma$ to itself.

If $S_{j}$ is a connected component of $S$, let $\dot{S}_{j}$ be the punctured surface obtained by removing all points in the set $(\{p\} \cup \Delta \cup N) \cap S_{j}$. Note that the stability condition implies $\chi\left(\dot{S}_{j}^{D}\right)<0$. There is a natural embedding $\dot{S}_{j} \hookrightarrow \overline{\mathbf{S}}_{r} \backslash \Theta_{\Delta, N}$, which we use to define the sequence of complex structures $\varphi_{k}^{*} j_{k}$ and metrics $\varphi_{k}^{*} h_{k}$ on $\dot{S}_{j}$. Then passing to a subsequence, we have $\varphi_{k}^{*} j_{k} \rightarrow j$ and $\varphi_{k}^{*} h_{k} \rightarrow h$ in $C_{\text {loc }}^{\infty}$ on $\dot{S}_{j}$, where $h$ is the Poincaré metric for $\left(\dot{S}_{j}, j\right)$. Since $d \tilde{u}_{k}$ is uniformly bounded on compact subsets, we can then find constants $c_{k}^{j} \in \mathbb{R}$ such that

$$
\tilde{v}_{k}^{j}=\left(b_{k}^{j}, v_{k}^{j}\right)=\left.\left(a_{k}+c_{k}^{j}, u_{k}\right) \circ \varphi_{k}\right|_{\dot{S}_{j}}:\left(\dot{S}_{j}, \varphi_{k}^{*} j_{k}\right) \rightarrow\left(\mathbb{R} \times S^{3}, \widetilde{J}_{k}\right)
$$

is a sequence of pseudoholomorphic maps satisfying the appropriate boundary conditions and a uniform $C^{1}$-bound. Thus $\tilde{v}_{k}^{j}$ has a $C_{\text {loc }}^{\infty}$-convergent subsequence

$$
\widetilde{v}_{k}^{j} \rightarrow \widetilde{v}^{j}=\left(b^{j}, v^{j}\right): \dot{S}_{j} \rightarrow \mathbb{R} \times S^{3},
$$

where $\widetilde{v}^{j}$ satisfies $T \widetilde{v}^{j} \circ j=\widetilde{J}_{\infty} \circ T \widetilde{v}^{j}$. Due to the uniform energy bound for $\tilde{u}_{k}$, we see also that $E_{\infty}\left(\widetilde{v}^{j}\right)<\infty$. Repeating this process for every component $S_{j} \subset S$, we obtain a set of $\widetilde{J}_{\infty}$-holomorphic maps

$$
\begin{gathered}
\widetilde{v}^{1}: \dot{S}_{1} \rightarrow \mathbb{R} \times S^{3}, \\
\vdots \\
\widetilde{v}^{N}: \dot{S}_{N} \rightarrow \mathbb{R} \times S^{3} .
\end{gathered}
$$

Our main goal now is to show that $\mathbf{S}$ is actually a smooth Riemann surface with boundary, ie $\Delta$ and $N$ are empty sets and $S$ has only one component. Then the set of 
solutions above reduces to a single solution $\tilde{u}_{\infty}: \dot{\Sigma} \rightarrow \mathbb{R} \times M$, which we must show is positively asymptotic to $P_{\infty}$ at the puncture. As with the bubbling off arguments in the previous section, these results will follow mainly from topological considerations.

Recall from Remark 3.1 that our notation allows some of the components $K_{i}$ and $K_{j}$ of $K$ to be identical; in particular, topological considerations require a given component $K_{i} \subset K$ to repeat $n$ times in the list $K_{1}, \ldots, K_{m}$ if $\operatorname{lk}\left(K_{i}, P_{\infty}\right)=n$. The lists of components $N=N_{1} \cup \cdots \cup N_{m}$ and $L=L_{1} \cup \cdots \cup L_{m}$ are then defined with similar repetitions. If $\gamma_{1}, \ldots, \gamma_{m}$ are the connected components of $\partial \Sigma$ (not repeated), then the oriented loop $u_{k}\left(\gamma_{j}\right)$ is a meridian on $L_{j}=\partial N_{j}$ with $\operatorname{lk}\left(u_{k}\left(\gamma_{j}\right), K_{j}\right)=-1$. Thus the linking number $\operatorname{lk}\left(K_{j}, P_{\infty}\right)$ is precisely the number of distinct components of $\partial \Sigma$ mapped into the same torus $L_{j}$, and we have also $\operatorname{lk}\left(u_{k}\left(\gamma_{j}\right), K\right)=-1$ since $u_{k}\left(\gamma_{j}\right)$ is unlinked with all components of $K$ that are distinct from $K_{j}$. Adding this up for all $\gamma_{j} \subset \partial \Sigma$, we see that the expression

$$
-\operatorname{lk}\left(u_{k}(\partial \Sigma), K\right)
$$

counts the connected components of $\partial \Sigma$. Also, the map $u_{k}$ realizes a homology $\partial\left[u_{k}\right]=\left[P_{\infty}\right]+\left[u_{k}(\partial \Sigma)\right]$ in $S^{3} \backslash K$, which gives the useful formula

$$
\operatorname{lk}\left(K_{j}, P_{\infty}\right)=-\operatorname{lk}\left(K_{j}, u_{k}(\partial \Sigma)\right) .
$$

In light of this topological setup, $u_{k}$ extends to a smooth map

$$
\bar{u}_{k}: \mathbb{C} \rightarrow \mathbb{R} \times S^{3}
$$

which satisfies $T \bar{u}_{k} \circ j_{k}=\widetilde{J}_{k} \circ T \bar{u}_{k}$ in $\dot{\Sigma}=\mathbb{C} \backslash\left(\mathcal{D}_{1} \cup \cdots \cup \mathcal{D}_{m}\right) \subset \mathbb{C}$, and maps each of the disks $\mathcal{D}_{j}$ into $N_{j}$. We may assume that $\left.\bar{u}_{k}\right|_{\mathcal{D}_{j}}$ has a single transverse positive intersection with $K_{j}$.

Let $S_{1} \subset S$ be the connected component that contains the marked point $p$.

Claim $\widetilde{v}^{1}$ is positively asymptotic to $P_{\infty}$ at $p$.

If the puncture is removable, then we can find an oriented circle $C \subset \dot{S}_{1}$ winding clockwise around $p$ such that $v^{1}(C)$ lies in an arbitrarily small neighborhood of some point in $S^{3} \backslash K$. Then this neighborhood also contains $v_{k}^{1}(C)=u_{k}\left(\varphi_{k}(C)\right)$ for sufficiently large $k$, and $\varphi_{k}(C)$ is a large circle in $\mathbb{C}$, bounding a simply connected region $\Omega$. One can then define a smooth map

$$
\widehat{u}_{k}: \mathbb{C} \rightarrow S^{3} \backslash K
$$

which matches $u_{k}$ outside of $\Omega$, so that the loops $\hat{u}_{k}\left(\partial \mathbb{D}_{R}\right)$ approach $P_{\infty}$ as $R \rightarrow \infty$. This implies that for any component $K_{j} \subset K, \operatorname{lk}\left(P_{\infty}, K_{j}\right)=0$, a contradiction. 
If $p$ is a nonremovable puncture and $\widetilde{v}^{1}$ is asymptotic to an orbit $P$ that is geometrically distinct from $P_{\infty}$, we similarly find a large clockwise oriented circle $\varphi_{k}(C) \subset \mathbb{C}$, bounding a region $\Omega$, such that $u_{k}\left(\varphi_{k}(C)\right)$ is close to $P$. Then the existence of the map $\left.\bar{u}_{k}\right|_{\Omega}: \Omega \rightarrow S^{3} \backslash P_{\infty}$ implies $\operatorname{lk}\left(P, P_{\infty}\right)=0$, and this is impossible. The alternative is that $P$ could be an $n$-fold cover of $P_{\infty}$ for some integer $n \neq 0$. (Negative $n$ would again mean the puncture is negative.) But then restricting $u_{k}$ to the region outside of $\Omega$ gives a homotopy of $u_{k}\left(\varphi_{k}(C)\right)$ to $P_{\infty}$ in $S^{3} \backslash K$, implying that for any component $K_{j} \subset K$,

$$
n \cdot \operatorname{lk}\left(P_{\infty}, K_{j}\right)=\operatorname{lk}\left(u_{k} \circ \varphi_{k}(C), K_{j}\right)=\operatorname{lk}\left(P_{\infty}, K_{j}\right),
$$

so $n=1$. This proves the claim.

With the asymptotic behavior at $p$ understood, it remains to prove that $\mathbf{S}$ has no double points or unpaired nodes. Note that it suffices to prove this for the component $S_{1} \subset S$. We shall set up the discussion in a slightly more general way than is immediately necessary, since it will also be useful for the degeneration argument in the next section.

First some notation. The $m$ connected components of $\partial \Sigma$ are denoted

$$
\partial \Sigma=\gamma_{1} \cup \cdots \cup \gamma_{m}
$$

and let us write the components of $\partial S_{1}$ as

$$
\partial S_{1}=\alpha_{1} \cup \cdots \cup \alpha_{s} .
$$

Note that $m \geq 2$ by assumption, but $\partial S_{1}$ could conceivably be empty. Assume $S_{1}$ has a (possibly empty) set of unpaired nodes

$$
N \cap S_{1}=\left\{w_{1}, \ldots, w_{\ell}\right\},
$$

interior double points

$$
\Delta \cap \text { int } S_{1}=\left\{z_{1}, \ldots, z_{q}\right\},
$$

and boundary double points

$$
\Delta \cap \partial S_{1} \supset \Delta \cap \alpha_{j}=\left\{\zeta_{j}^{1}, \ldots, \zeta_{j}^{r_{j}}\right\} \text { for } j=1, \ldots, s,
$$

where we are regarding $\Delta$ for the moment as a set of points in $S$ rather than pairs of points. We know from Theorem 3.12 that $\widetilde{v}^{1}$ extends smoothly over each boundary double point $\zeta_{j}^{i} \in \Delta \cap \partial S_{1}$, and at each $z_{j} \in \Delta \cap$ int $S_{1}$ and $w_{j} \in N \cap S_{1}, \widetilde{v}_{1}$ either has a removable singularity or is asymptotic to some periodic orbit of $X_{\infty}$.

Let $\bar{S}_{1}$ denote the compact surface with piecewise smooth boundary obtained from $S_{1} \backslash\left((\Delta \cup N) \cap S_{1}\right)$ by replacing the interior punctures $z_{j}, w_{j}$ with circles at infinity $\delta_{z_{j}}, \delta_{w_{j}}$ and the boundary punctures $\zeta_{j}^{i}$ with arcs at infinity $\delta_{\zeta_{j}^{i}}$. Each component 
$\alpha_{j} \subset \partial S_{1}$ then gives rise to a piecewise smooth circular component $\bar{\alpha}_{j} \subset \partial \bar{S}_{1}$. There is a natural map $\bar{S}_{1} \rightarrow \overline{\mathbf{S}}_{r}$, which is an inclusion except possibly on $\partial \bar{S}_{1}$, where two distinct circles $\delta_{z_{j}}$ or arcs $\delta_{\xi_{j}^{i}}$ may have the same image; this corresponds to the identification of double points in a pair. Since $\overline{\mathbf{S}}_{r}$ is diffeomorphic to

$$
\Sigma=\mathbb{C} \cup\{\infty\} \backslash\left(\mathcal{D}_{1} \cup \cdots \cup \mathcal{D}_{m}\right),
$$

we can visualize $\overline{\mathbf{S}}_{r} \backslash\{p\}$ as the plane with a finite set of disks removed. An example of this is shown in Figure 6. Here we settle on the convention that the circles $\delta_{z_{j}}$ are always oriented as components of $\partial S_{1}$. Thus they appear as embedded loops winding clockwise in the plane, and each encloses a bounded region which may contain some of the disks $\mathcal{D}_{i}$. Let $m_{j}$ be the number of such disks enclosed by $\delta_{z_{j}}$. Similarly, for $j=1, \ldots, s$, denote by $\widehat{m}_{j}$ the number of disks in the compact region enclosed by $\bar{\alpha}_{j}$; this number is always at least 1 . Figure 6 shows a compact subset of $\overline{\mathbf{S}}_{r}$ which contains the entire boundary of $\bar{S}_{1}$. Here the closure of the white area is $\bar{S}_{1}$, and the lightly shaded regions constitute the rest of $\overline{\mathbf{S}}_{r}$, while the darkly shaded regions are the disks $\mathcal{D}_{j}$.

The integers defined above are related by

$$
m=\ell+\sum_{j=1}^{q} m_{j}+\sum_{j=1}^{s} \widehat{m}_{j}
$$

and as remarked already,

$$
\widehat{m}_{j} \geq 1 \text { for all } j=1, \ldots, s .
$$

There are also constraints imposed by the stability condition for each component of $S$ : the double of $\dot{S}_{1}$ must have negative Euler characteristic, thus

$$
2(s+q+\ell)+\sum_{j=1}^{s} r_{j}>2,
$$

and applying similar reasoning to the portions of $\overline{\mathbf{S}}_{r}$ inside the loops $\delta_{z_{j}}$, we have

$$
m_{j} \geq 2 \text { for all } j=1, \ldots, q \text {. }
$$

We now transfer this picture onto $\dot{\Sigma}$ via the diffeomorphism

$$
\varphi_{k}: \overline{\mathbf{S}}_{r} \backslash\{p\} \rightarrow \dot{\Sigma}
$$

for large $k$ (see Figure 7). For $j=1, \ldots, q$, denote by $\partial_{j} \Sigma$ the $m_{j}$ components of $\partial \Sigma$ that are enclosed within $\varphi_{k}\left(\delta_{z_{j}}\right)$, and for $j=1, \ldots, s$ let $\widehat{\partial}_{j} \Sigma$ be the $\widehat{m}_{j}$ components 


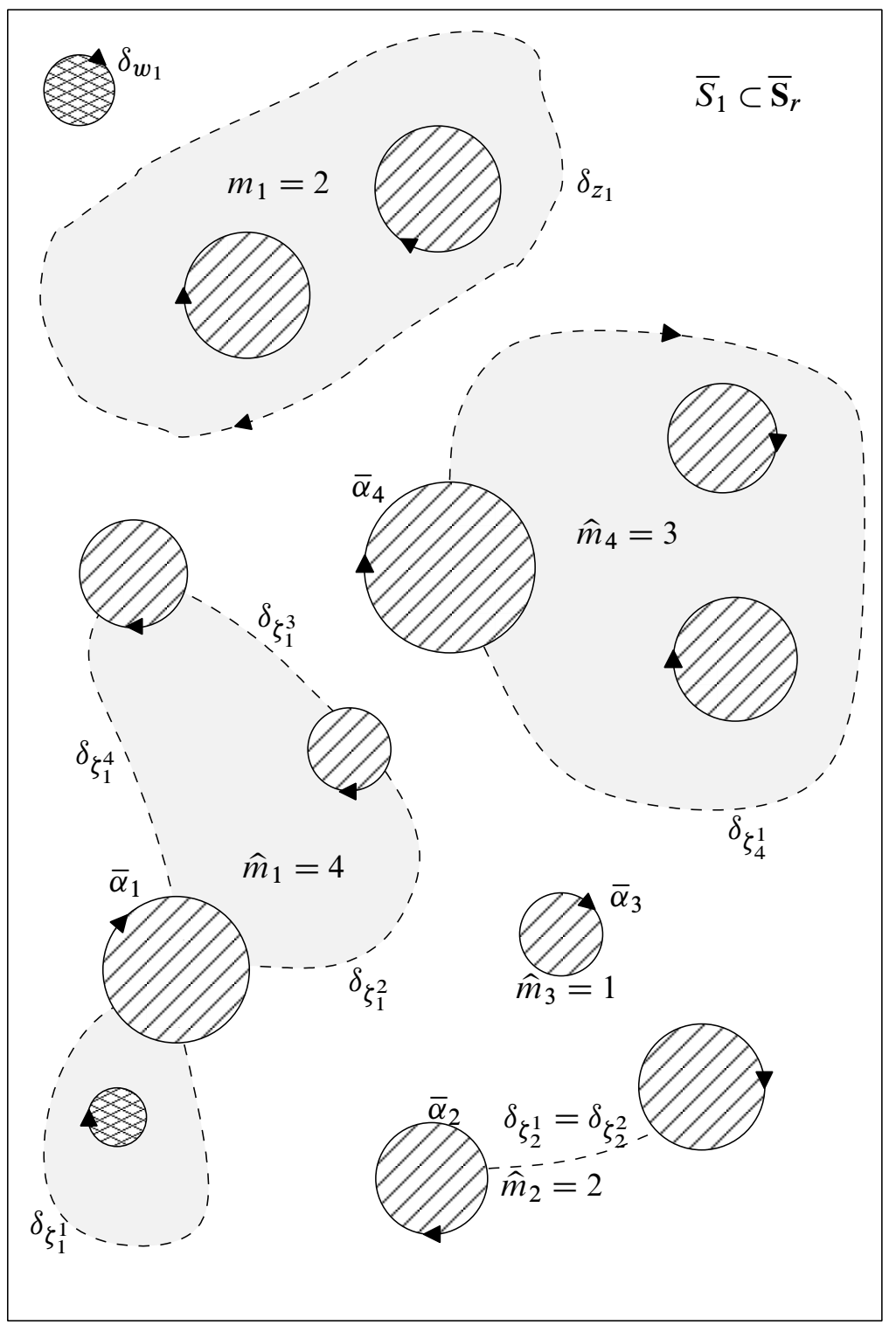

Figure 6: A compact subset of $\overline{\mathbf{S}}_{r}$ showing the piecewise smooth boundary of $\partial \bar{S}_{1}$. Here we assume $\partial S_{1}$ has four components $\alpha_{1}, \ldots, \alpha_{4}, S_{1}$ has one interior double point $\Delta \cap$ int $S_{1}=\left\{z_{1}\right\}$, seven boundary double points $\Delta \cap \alpha_{1}=\left\{\zeta_{1}^{1}, \ldots, \zeta_{1}^{4}\right\}, \Delta \cap \alpha_{2}=\left\{\zeta_{2}^{1}, \zeta_{2}^{2}\right\}, \Delta \cap \alpha_{3}=\varnothing, \Delta \cap \alpha_{4}=\left\{\zeta_{4}^{1}\right\}$, and one unpaired node $N \cap S_{1}=\left\{w_{1}\right\}$. 
in the closed region bounded by $\varphi_{k}\left(\bar{\alpha}_{j}\right)$. Now for each component $\alpha_{j} \subset \partial S_{1}$, we define a perturbed loop $\alpha_{j}^{\prime} \subset$ int $S_{1}$ which misses the double points. The images $\varphi_{k}\left(\alpha_{j}^{\prime}\right) \subset \dot{\Sigma}$ are represented as dotted loops in Figure 7; each encloses a bounded region that contains $\hat{\partial}_{j} \Sigma$. Similarly, for each interior double point $z_{j}$ we choose a perturbed loop $C_{j} \subset$ int $\bar{S}_{1}$ near $\delta_{z_{j}}$, so $\varphi_{k}\left(C_{j}\right)$ encloses $\partial_{j} \Sigma$. Define also the loops $\beta_{j} \subset$ int $\bar{S}_{1}$ as perturbations of $\delta_{w_{j}}$ for unpaired nodes $w_{j} \in N \cap S_{1}$ : thus each $\varphi_{k}\left(\beta_{j}\right)$ encloses a unique connected component $\gamma_{g(j)} \subset \partial \Sigma$. Observe that $\partial \Sigma$ is now the disjoint union

$$
\partial \Sigma=\left(\bigcup_{j=1}^{q} \partial_{j} \Sigma\right) \cup\left(\bigcup_{j=1}^{s} \widehat{\partial}_{j} \Sigma\right) \cup\left(\bigcup_{j=1}^{\ell} \gamma_{g(j)}\right) .
$$

The images under $\varphi_{k}$ of the various perturbed loops are shown with dotted lines in Figure 7.

From this picture we can deduce some topological facts about the behavior of $v^{1}: \dot{S}_{1} \rightarrow$ $S^{3}$ at its boundary and punctures. For a component $\alpha_{j} \subset \partial S_{1}$, we have $v^{1}\left(\alpha_{j}\right) \subset L_{f(j)}$ for some $f(j) \in\{1, \ldots, m\}$, and we can assume $u_{k} \circ \varphi_{k}\left(\alpha_{j}^{\prime}\right)$ is $C^{0}$-close to $v^{1}\left(\alpha_{j}\right)$. Then restricting $u_{k}$ to the bounded region inside $\varphi_{k}\left(\alpha_{j}^{\prime}\right)$ realizes a homology

$$
\partial\left[u_{k}\right]=-\left[u_{k} \circ \varphi_{k}\left(\alpha_{j}^{\prime}\right)\right]+\left[u_{k}\left(\hat{\partial}_{j} \Sigma\right)\right]
$$

in both $S^{3} \backslash P_{\infty}$ and $S^{3} \backslash K$. This implies

$$
\operatorname{lk}\left(u_{k} \circ \varphi_{k}\left(\alpha_{j}^{\prime}\right), P_{\infty}\right)=\operatorname{lk}\left(u_{k}\left(\hat{\partial}_{j} \Sigma\right), P_{\infty}\right)=0,
$$

and thus

$$
\operatorname{lk}\left(v^{1}\left(\alpha_{j}\right), P_{\infty}\right)=0
$$

This means $v^{1}\left(\alpha_{j}\right)$ covers a meridian on $L_{f(j)}$, and its homotopy class can be deduced exactly via the linking number with $K$ :

$$
\operatorname{lk}\left(v^{1}\left(\alpha_{j}\right), K\right)=\operatorname{lk}\left(u_{k} \circ \varphi_{k}\left(\alpha_{j}^{\prime}\right), K\right)=\operatorname{lk}\left(u_{k}\left(\widehat{\partial}_{j} \Sigma\right), K\right)=-\widehat{m}_{j} .
$$

Since $v^{1}\left(\alpha_{j}\right)$ is only linked with one component of $K$,

$$
\operatorname{lk}\left(v^{1}\left(\alpha_{j}\right), K_{f(j)}\right)=-\widehat{m}_{j}
$$

Turning our attention next to the unpaired nodes, let us assume there is a simply covered orbit $P_{j} \subset S^{3}$ of $X_{\infty}$ such that $\widetilde{v}^{1}$ is asymptotic to an $\left|n_{j}\right|$-fold cover of $P_{j}$ at $w_{j} \in N \cap S_{1}$, for some $n_{j} \in \mathbb{Z}$. Again, we're using the convention that the sign of $n_{j}$ matches the sign of the puncture at $w_{j}$, and we set $n_{j}=0$ if the puncture is 


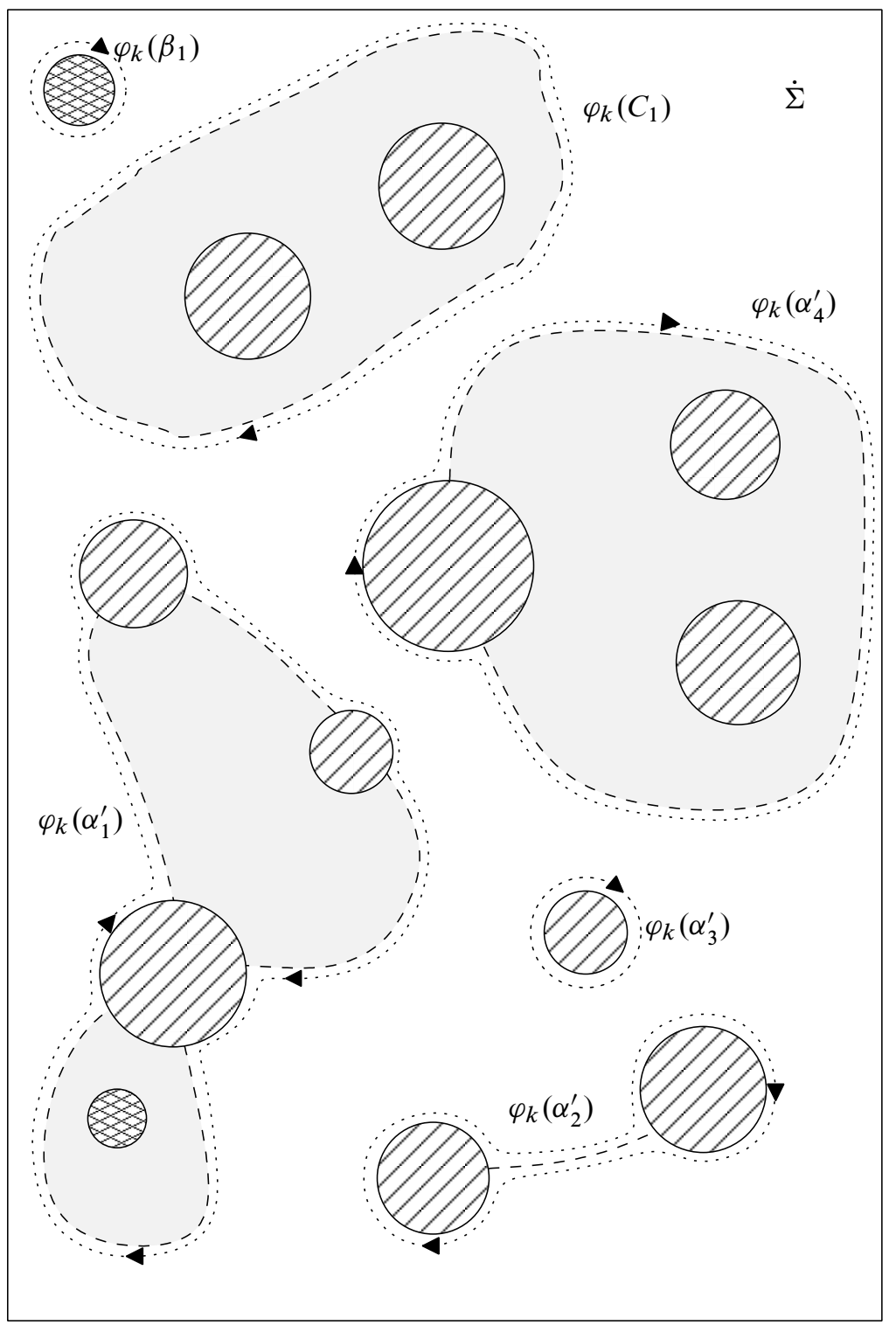

Figure 7: The image of Figure 6 under $\varphi_{k}: \overline{\mathbf{S}}_{r} \backslash\{p\} \rightarrow \dot{\Sigma}$, showing the perturbed loops $\alpha_{1}^{\prime}, \ldots, \alpha_{4}^{\prime}, \beta_{1}$ and $C_{1}$ as dotted lines.

removable (in which case it doesn't matter what $P_{j}$ is). Now, restricting $u_{k}$ to the 
region between $\gamma_{g(j)}$ and $\varphi_{k}\left(\beta_{j}\right)$, we have a homology

$$
\partial\left[u_{k}\right]=\left[u_{k}\left(\gamma_{g(j)}\right)\right]-\left[u_{k} \circ \varphi_{k}\left(\beta_{j}\right)\right],
$$

in both $S^{3} \backslash P_{\infty}$ and $S^{3} \backslash K$, and we can assume $\left[u_{k} \circ \varphi_{k}\left(\beta_{j}\right)\right]$ is homologous to $n_{j}\left[P_{j}\right]$. Thus for every component $K_{i} \subset K$,

$$
n_{j} \operatorname{lk}\left(P_{j}, K_{i}\right)=\operatorname{lk}\left(u_{k}\left(\gamma_{g(j)}\right), K_{i}\right)
$$

Adding these up for all components of $K$, we find

$$
n_{j} \operatorname{lk}\left(P_{j}, K\right)=-1,
$$

implying that the puncture is nonremovable and the orbit is simply covered. If $P_{j}=P_{\infty}$ this gives $n_{j} m=-1$, which cannot be true since $m \geq 2$ by assumption. Thus $P_{j}$ is geometrically distinct from $P_{\infty}$, and using the homology in $S^{3} \backslash P_{\infty}$, we have $n_{j} \operatorname{lk}\left(P_{j}, P_{\infty}\right)=\operatorname{lk}\left(u_{k}\left(\gamma_{g}(j)\right), P_{\infty}\right)=0$, implying

$$
\operatorname{lk}\left(P_{j}, P_{\infty}\right)=0 .
$$

We can reach similar conclusions about the behavior of $\widetilde{v}^{1}$ at an interior double point $z_{j} \in \Delta \cap \operatorname{int} S_{1}$. Using the same convention as above, assume $v^{1}$ approaches an $\left|n_{j}^{\prime}\right|-$ fold cover of some simply covered orbit $P_{j}^{\prime}$ at $z_{j}$. Then we may assume $\left[u_{k} \circ \varphi_{k}\left(C_{j}\right)\right]$ is homologous to $n_{j}^{\prime}\left[P_{j}^{\prime}\right]$, and by restricting $u_{k}$ over the bounded region inside $\varphi_{k}\left(C_{j}\right)$,

$$
\partial\left[u_{k}\right]=\left[u_{k}\left(\partial_{j} \Sigma\right)\right]-\left[u_{k} \circ \varphi_{k}\left(C_{j}\right)\right]
$$

in both $S^{3} \backslash K$ and $S^{3} \backslash P_{\infty}$. This implies for all components $K_{i} \subset K$,

$$
n_{j}^{\prime} \operatorname{lk}\left(P_{j}^{\prime}, K_{i}\right)=\operatorname{lk}\left(u_{k}\left(\partial_{j} \Sigma\right), K_{i}\right),
$$

and summing this over the components of $K$, we have

$$
n_{j}^{\prime} \operatorname{lk}\left(P_{j}^{\prime}, K\right)=-m_{j} \leq-2,
$$

so $n_{j}^{\prime}$ cannot be zero, ie the puncture is not removable. If $P_{j}^{\prime}=P_{\infty}$, we have $n_{j}^{\prime} m=-m_{j}$, then $m_{j} \leq m$ implies $n_{j}^{\prime}=-1$ and $m=m_{j}$. But this contradicts the stability assumption; indeed, combining (17), (19) and (16), we find $q=1$ and $s=\ell=0$, violating (18). Therefore $P_{j}^{\prime}$ is geometrically distinct from $P_{\infty}$, and the homology in $S^{3} \backslash P_{\infty}$ gives $n_{j}^{\prime} \operatorname{lk}\left(P_{j}^{\prime}, P_{\infty}\right)=\operatorname{lk}\left(u_{k}\left(\partial_{j} \Sigma\right), P_{\infty}\right)=0$, thus

$$
\operatorname{lk}\left(P_{j}^{\prime}, P_{\infty}\right)=0 .
$$

At this point all the vital ingredients are in place.

Claim $N \cap S_{1}$ and $\Delta \cap S_{1}$ are empty. 
If $w_{j} \in N \cap S_{1}$, then $v^{1}$ is asymptotic to a periodic orbit $P_{j}$ which is geometrically distinct from $P_{\infty}$, and is also unlinked with it, by (23). But we have assumed there is no such orbit, therefore $N \cap S_{1}=\varnothing$. The same argument proves $\Delta \cap \operatorname{int} S_{1}=\varnothing$, using (25).

It remains only to exclude double points on the boundary. We now can assume that $\partial S_{1} \neq \varnothing$ and the only puncture of $\widetilde{v}^{1}$ is at $p$, where it is positively asymptotic to $P_{\infty}$. By assumption, there is a trivialization $\Phi_{\infty}$ of $\left.\left(v^{1}\right)^{*} \xi_{\infty}\right|_{M}$ for which $\mu_{\mathrm{CZ}}^{\Phi_{\infty}}\left(P_{\infty}\right)=3$ and, using (21) and the fact that $v^{1}\left(\alpha_{j}\right)$ covers a meridian for each component $\alpha_{j} \subset$ $\partial S_{1}$, the Maslov index along $\alpha_{j}$ is $2 \operatorname{lk}\left(v^{1}\left(\alpha_{j}\right), K_{f(j)}\right)=-2 \widehat{m}_{j}$. Thus we compute $\mu\left(\widetilde{v}^{1}\right)=3-2 \sum_{j=1}^{s} \widehat{m}_{j}$, and

$$
\operatorname{ind}\left(\widetilde{v}^{1}\right)=\mu\left(\widetilde{v}^{1}\right)-\chi\left(\dot{S}_{1}\right)+s=3-2 \sum_{j=1}^{s} \widehat{m}_{j}-(1-s)+s=2+2 \sum_{j=1}^{s}\left(1-\widehat{m}_{j}\right)
$$

The $\omega_{\infty}$-energy of $\widetilde{v}^{1}$ is clearly nonzero since $v^{1}\left(\partial S_{1}\right)$ and the image of $v^{1}$ near $p$ cannot belong to the same orbit. Thus Theorem 2.4 gives

$$
0 \leq 2 \operatorname{wind}_{\pi}\left(\widetilde{v}^{1}\right) \leq 2 \sum_{j=1}^{s}\left(1-\widehat{m}_{j}\right)
$$

Since $\widehat{m}_{j} \geq 1$ for all $j$, the right hand side of this expression is never positive, and is zero if and only if $\widehat{m}_{j}=1$ for all $j$. This excludes situations such as $\bar{\alpha}_{1}$ and $\bar{\alpha}_{2}$ in Figure 6, where double points give rise to arcs that connect two distinct disks. All the arcs in $\delta_{\zeta_{j}^{i}} \subset \partial \bar{S}_{1}$ must therefore begin and end on the same circle, enclosing a region of the plane as with $\bar{\alpha}_{4}$ in the figure. But now the stability condition requires this enclosed region to have negative Euler characteristic after doubling, which can only be true if it contains at least one disk, contradicting the fact that $\widehat{m}_{j}=1$. We conclude that there are no such arcs $\delta_{\zeta_{j}^{i}}$, and hence no double points $\zeta_{j}^{i} \in \Delta \cap \partial S_{1}$.

It follows now that $\mathbf{S}$ has no double points or unpaired nodes at all, thus the convergence $\left(\Sigma, j_{k},\{\infty\}\right) \rightarrow(S, j,\{p\}, \Delta, N)$ simply means there are diffeomorphisms $\varphi_{k}: S \rightarrow$ $\Sigma$ such that $\varphi_{k}(p)=\infty$ and $\varphi_{k}^{*} j_{k} \rightarrow j$ in $C^{\infty}(S)$. Then after $\mathbb{R}$-translation, $\tilde{u}_{k} \circ \varphi_{k} \rightarrow \widetilde{v}^{1}$ in $C_{\mathrm{loc}}^{\infty}\left(S \backslash\{p\}, \mathbb{R} \times S^{3}\right)$, and $\widetilde{v}^{1}$ has the same asymptotic limit as $\tilde{u}_{k}$. This completes the proof of Proposition 3.21.

\subsection{Degeneration at the boundary}

The proof of $C_{\text {loc }}^{\infty}$-convergence in Theorem 3.5 uses many of the same arguments as Theorem 3.4, so we will not repeat these in any detail, but rather emphasize the aspects 
that change when the new orbits appear at $\partial M$ in the limit. As before, it's convenient to treat the stable and non-stable cases separately.

The non-stable case The assumptions of Theorem 3.5 require that $\partial \Sigma$ be nonempty, so the only non-stable case to consider is $m=1$ : then $K$ is a knot with $\operatorname{lk}\left(P_{\infty}, K\right)=1$, and we may assume $\left(\dot{\Sigma}, j_{k}\right)=(\mathbb{C} \backslash \mathcal{D}, i)$ where $\mathcal{D}=$ int $\mathbb{D}$. It will be convenient to use the biholomorphic map

$$
\psi: \mathbb{R} \times S^{1} \rightarrow \mathbb{C} \backslash\{0\}:(s, t) \mapsto e^{2 \pi(s+i t)}
$$

and consider the sequence of $\widetilde{J}_{k}$-holomorphic half-cylinders

$$
\tilde{v}_{k}=\left(b_{k}, v_{k}\right)=\tilde{u}_{k} \circ \psi:[0, \infty) \times S^{1} \rightarrow \mathbb{R} \times S^{3},
$$

with $v_{k}\left(\{s\} \times S^{1}\right) \rightarrow P_{\infty}$ as $s \rightarrow \infty$. We claim $\left|d \widetilde{v}_{k}\right|$ is uniformly bounded. The proof is almost identical to what was done in Proposition 3.20: a sequence $z_{k}$ with $\left|d \widetilde{v}_{k}\left(z_{k}\right)\right| \rightarrow \infty$ gives rise to a non-constant finite energy plane or disk. A disk is impossible for the same reasons as before: its boundary would have to be contractible on $L$, leading to the conclusion that the map is constant. A plane cannot be asymptotic to any cover of $P_{\infty}$ or any orbit that is linked with it. The only new feature is that $a$ priori the plane could be asymptotic to one of the orbits on $L$, but this would imply that $v_{k}$ intersects $K$ for large $k$, and is thus also excluded.

Pick an open neighborhood $\mathcal{U}$ of $P_{\infty}$ in $M$, small enough so that its closure does not intersect $L$. Then define

$$
s_{k}=\min \left\{s \in[0, \infty) \mid v_{k}\left((s, \infty) \times S^{1}\right) \subset \mathcal{U}\right\} .
$$

\section{Claim $s_{k} \rightarrow \infty$.}

If not, there is a subsequence for which $s_{k} \rightarrow s_{\infty} \in[0, \infty)$ and (in light of the gradient bound), there are real numbers $c_{k}$ such that $\left(b_{k}+c_{k}, v_{k}\right)$ is $C_{\text {loc }}^{\infty}$-convergent to a $\widetilde{J}_{\infty}$-holomorphic half-cylinder

$$
\tilde{v}=(b, v):[0, \infty) \times S^{1} \rightarrow \mathbb{R} \times S^{3}
$$

with finite energy. Observe that $v\left(\{0\} \times S^{1}\right)$ is a meridian on $L$. Then if the puncture of $\widetilde{v}$ is removable, $v$ extends to a disk which must intersect $K$, implying that some part of the image of $v_{k}$ lies inside the solid torus $N$ for sufficiently large $k$, a contradiction.

Since $\infty$ is not a removable puncture, denote by $P$ its asymptotic orbit. Now the usual linking arguments imply

$$
\operatorname{lk}(P, K)=\operatorname{lk}\left(P_{\infty}, K\right)=1
$$


and if $P$ and $P_{\infty}$ are geometrically distinct,

$$
\operatorname{lk}\left(P, P_{\infty}\right)=0 .
$$

This leaves only two possibilities: $P$ is $P_{\infty}$ (positive puncture, simply covered) or it is a simply covered orbit on $L$ (negative puncture). In the latter case, the fact that $\omega_{\infty}$ is exact and vanishes on $L$ implies $E_{\omega_{\infty}}(\widetilde{v})=0$, thus the image of $v$ is contained in a periodic orbit, and therefore in $L$. But this gives a contradiction, because $v\left(\left\{s_{\infty}\right\} \times S^{1}\right)$ is in the closure of $\mathcal{U}$, which is disjoint from $L$.

There remains the possibility that $\widetilde{v}$ is positively asymptotic to $P_{\infty}$. But then $\widetilde{v}$ has precisely the same asymptotic and boundary conditions as $\widetilde{v}_{k}$, hence ind $(\widetilde{v})=2$ and Theorem 2.4 gives $\operatorname{wind}_{\pi}(\widetilde{v})=0$. This implies $v$ is transverse to $X_{\infty}$, which is impossible at $\{0\} \times S^{1}$ because both the image of $v$ and the orbits of $X_{\infty}$ on $L$ are meridians. This contradiction proves the claim that $s_{k} \rightarrow \infty$.

With this established, define a sequence

$$
\widetilde{w}_{k}=\left(\beta_{k}, w_{k}\right):\left[-s_{k}, \infty\right) \times S^{1} \rightarrow \mathbb{R} \times S^{3}
$$

by $\widetilde{w}_{k}(s, t)=\widetilde{v}_{k}\left(s+s_{k}, t\right)$. Then a subsequence of $\left(\beta_{k}+c_{k}, w_{k}\right)$ converges in $C_{\text {loc }}^{\infty}\left(\mathbb{R} \times S^{1}, \mathbb{R} \times S^{3}\right)$ to a $\widetilde{J}_{\infty}$-holomorphic finite energy cylinder

$$
\tilde{w}_{\infty}=\left(\beta_{\infty}, w_{\infty}\right): \mathbb{R} \times S^{1} \rightarrow \mathbb{R} \times S^{3} .
$$

The loop $\gamma:=w_{\infty}\left(\{0\} \times S^{1}\right)$ is now the uniform limit of $v_{k}\left(\left\{s_{k}\right\} \times S^{1}\right)$, and the usual arguments show that

$$
\operatorname{lk}\left(\gamma, P_{\infty}\right)=0 \quad \text { and } \quad \operatorname{lk}(\gamma, K)=1,
$$

thus $\widetilde{w}_{\infty}$ cannot be a constant map. If $E_{\omega_{\infty}}\left(\widetilde{w}_{\infty}\right)=0$, then these linking conditions and the fact that $\gamma$ is in the closure of $\mathcal{U}$ imply that $\widetilde{w}_{\infty}$ parametrizes $\mathbb{R} \times P_{\infty}$. However, there exists a sequence $\left(s_{k}^{\prime}, t_{k}^{\prime}\right)$ with $s_{k}^{\prime}<s_{k}$ and $s_{k}-s_{k}^{\prime} \rightarrow 0$ such that $v_{k}\left(s_{k}^{\prime}, t_{k}^{\prime}\right) \notin \mathcal{U}$, implying that $\gamma$ also meets the boundary of $\mathcal{U}$, a contradiction. Therefore $E_{\omega_{\infty}}\left(\widetilde{w}_{\infty}\right)>$ 0 .

We shall now show that both punctures of $\widetilde{w}_{\infty}$ are positive and asymptotic to the appropriate orbits. If both are removable, we obtain a nonconstant holomorphic sphere of index -2 , contradicting Theorem 2.4 as before. If only one is removable, then we can define a smooth map of a disk into $S^{3} \backslash K$ sending the boundary to $\gamma$, implying the contradiction $\operatorname{lk}(\gamma, K)=0$. Now denote the two asymptotic orbits by $w_{\infty}\left(\{ \pm \infty\} \times S^{1}\right)=P_{ \pm}$. We find,

$$
\operatorname{lk}\left(P_{ \pm}, K\right)=\operatorname{lk}\left(P_{\infty}, K\right)=1,
$$


and if $P_{ \pm}$is geometrically distinct from $P_{\infty}$

$$
\operatorname{lk}\left(P_{ \pm}, P_{\infty}\right)=0 .
$$

Therefore each orbit $P_{ \pm}$is either $P_{\infty}$ or is contained in $L$, simply covered in either case. We can determine the sign of each puncture by comparing the orientations of $w_{\infty}\left(\{ \pm \infty\} \times S^{1}\right)$ with the orientations of the orbits. This allows four possibilities:

(i) $\quad P_{+}=P_{\infty}$ (positive puncture) and $P_{-}=P_{\infty}$ (negative puncture),

(ii) $P_{+} \subset L$ (negative puncture) and $P_{-} \subset L$ (positive puncture),

(iii) $P_{+}=P_{\infty}$ (positive puncture) and $P_{-} \subset L$ (positive puncture) and

(iv) $P_{+} \subset L$ (negative puncture) and $P_{-}=P_{\infty}$ (negative puncture).

Case (iv) is immediately excluded because both punctures can't be negative. Cases (i) and (ii) would both imply $E_{\omega_{\infty}}\left(\widetilde{w}_{\infty}\right)=0$, using again the fact that $\omega_{\infty}$ is exact and vanishes on $L$. We conclude that both punctures are positive, with $P_{+}=P_{\infty}$ and $P_{-} \subset L$.

To apply this result to the sequence $\tilde{u}_{k}$, define a sequence of diffeomorphisms

$$
\varphi_{k}: \mathbb{C} \backslash\{0\} \rightarrow \mathbb{C} \backslash \mathbb{D}
$$

such that $\varphi_{k}(z)=e^{2 \pi s_{k}} z$ for all $z$ with $|z| \geq 2 e^{-2 \pi s_{k}}$. Then observe that $\tilde{w}_{k} \circ$ $\psi^{-1}(z)=\widetilde{u}_{k} \circ \varphi_{k}(z)$ whenever $|z| \geq 2 e^{-2 \pi s_{k}}$, thus after $\mathbb{R}$-translation, a subsequence of $\tilde{u} \circ \varphi_{k}$ converges in $C_{\text {loc }}^{\infty}\left(\mathbb{C} \backslash\{0\}, \mathbb{R} \times S^{3}\right)$ to

$$
\tilde{u}_{\infty}=\tilde{w}_{\infty} \circ \psi^{-1}: \mathbb{C} \backslash\{0\} \rightarrow \mathbb{R} \times S^{3},
$$

which is asymptotic to $P_{\infty}$ at $\infty$ and an orbit on $L$ at 0 . Clearly also $\varphi_{k}^{*} i \rightarrow i$ in $C_{\text {loc }}^{\infty}\left(S^{2} \backslash\{0\}\right)$. We have thus proved $C_{\text {loc }}^{\infty}$-convergence for Theorem 3.5 in the case $\chi(\dot{\Sigma}) \geq 0$.

The stable case Now assume $\chi(\dot{\Sigma})<0$. The proof of Theorem 3.5 in this case will follow roughly the same sequence of steps as in Theorem 3.4, with a few important differences.

Step 1: Gradient bounds We begin by establishing a bound

$$
\left|d \tilde{u}_{k}(z)\right|_{k} \leq \frac{C}{\operatorname{injrad}_{k}(z)} .
$$

The proof is mostly the same as in Proposition 3.19. If a finite energy plane bubbles off, then it is asymptotic to an orbit $P$ which (for topological reasons) cannot be a 
cover of $P_{\infty}$, and $\operatorname{lk}\left(P, P_{\infty}\right)=0$. The only remaining alternative (which is new in this situation) is that $P$ is a meridian on one of the tori $L_{j}$, but this would imply $\operatorname{lk}\left(P, K_{j}\right) \neq 0$, so $u_{k}(\dot{\Sigma})$ would have to intersect $K_{j}$ for some large $k$. The argument excluding disk bubbles is the same as before.

As in the proof of Proposition 3.21, a subsequence of $\left(\Sigma, j_{k},\{\infty\}\right)$ converges to a stable nodal surface $\mathbf{S}=(S, j,\{p\}, \Delta, N)$. We again denote the connected components by $S=S_{1} \cup \cdots \cup S_{N}$ with corresponding punctured surfaces $\dot{S}_{j}$, choosing the labels so that $p \in S_{1}$. The gradient bound above implies that we can find constants $c_{k}^{j} \in \mathbb{R}$ such that

$$
\left.\left(a_{k}+c_{k}^{j}, u_{k}\right) \circ \varphi_{k}\right|_{\dot{S}_{j}} \rightarrow \widetilde{v}^{j}: \dot{S}_{j} \rightarrow \mathbb{R} \times S^{3}
$$

in $C_{\mathrm{loc}}^{\infty}\left(\dot{S}_{j}, \mathbb{R} \times S^{3}\right)$, where $T \widetilde{v}^{j} \circ j=\widetilde{J}_{\infty} \circ T \widetilde{v}^{j}$. Our main goal will be to show that $\mathbf{S}$ has no double points and no boundary, but does have $m$ unpaired nodes, one corresponding to each component of $\partial \Sigma$.

Step 2: Asymptotic behavior at $p$ The same arguments as in Proposition 3.21 show that $p$ is a nonremovable puncture for $\widetilde{v}^{1}: \dot{S}_{1} \rightarrow \mathbb{R} \times S^{3}$, and if $P$ is an asymptotic limit then either $P=P_{\infty}$ (simply covered) or $P$ is geometrically distinct from $P_{\infty}$, with $\operatorname{lk}\left(P, P_{\infty}\right)=0$. In the present context this last possibility implies that $P$ is an $n$-fold cover of some orbit $P_{1}$ on one of the tori $L_{i}$, with $\operatorname{lk}\left(P, K_{i}\right)=n \cdot \operatorname{lk}\left(P_{1}, K_{i}\right)=-n$. (As always, $n \neq 0$ and is negative if the puncture is negative.) Then we can choose a small circle $C$ about $p$ such that $u_{k}\left(\varphi_{k}(C)\right)$ is close to $P$ for some large $k$, and thus construct a homotopy from $P$ to $P_{\infty}$ through $S^{3} \backslash K$, implying

$$
\operatorname{lk}\left(P, K_{j}\right)=\operatorname{lk}\left(P_{\infty}, K_{j}\right)>0
$$

for each component $K_{j} \subset K$. The left hand side is 0 if $K_{j} \neq K_{i}$, so this alternative can only happen if $K$ is connected: in that case $-n=\operatorname{lk}\left(P_{\infty}, K\right)=m$, so $p$ is a negative puncture and $P$ is an $m$-fold cover of $P_{1}$. We shall use arguments similar to the proof of the non-stable case to show that this is also impossible.

Identify a punctured neighborhood of $p$ in $\dot{S}_{1}$ with the positive half-cylinder via a holomorphic embedding

$$
\psi:[0, \infty) \times S^{1} \hookrightarrow S_{1} \backslash\{p\},
$$

and define $\tilde{w}_{k}=\left(\beta_{k}, w_{k}\right)=\tilde{u}_{k} \circ \varphi_{k} \circ \psi:[0, \infty) \times S^{1} \rightarrow \mathbb{R} \times S^{3}$. These half-cylinders are $\tilde{J}_{k}$-holomorphic with the varying complex structures $\left(\varphi_{k} \circ \psi\right)^{*} j_{k}=\psi^{*} \varphi_{k}^{*} j_{k}$ on the domain, and they converge in $C_{\text {loc }}^{\infty}$ (possibly after translation in $\mathbb{R} \times M$ ) to $\tilde{v}^{1} \circ \psi$. Observe that $\varphi_{k}^{*} j_{k} \rightarrow j$ in $C^{\infty}$ on any compact neighborhood of $p$, thus $\psi^{*} \varphi_{k}^{*} j_{k} \rightarrow \psi^{*} j=i$ in $C^{\infty}\left([0, \infty) \times S^{1}\right)$, not just on compact subsets; this follows 
from Lemma 3.22 below. The familiar argument then establishes a uniform bound on $\left|d \widetilde{w}_{k}\right|$ over $[0, \infty) \times S^{1}$ : the alternative is that $\left|d \widetilde{w}_{k}\left(s_{k}, t_{k}\right)\right|$ blows up on some sequence with $s_{k} \rightarrow \infty$, in which case a $\widetilde{J}_{\infty}$-holomorphic finite energy plane bubbles off, leading to the usual contradictions.

Due to the asymptotic behavior of $\widetilde{v}^{1}$, there exists a sequence $s_{k} \rightarrow \infty$ such that $w_{k}\left(s_{k}, \cdot\right)$ converges in $C^{\infty}\left(S^{1}, S^{3}\right)$ to a negatively oriented $m$-fold cover of the orbit $P_{1}$. But the half-cylinders $\widetilde{w}_{k}$ are each asymptotic to $P_{\infty}$, thus we can (as in the stable case) pick a small open neighborhood $P_{\infty} \subset \mathcal{U} \subset M$ and define

$$
s_{k}^{\prime}=\min \left\{s \in[0, \infty) \mid w_{k}\left((s, \infty) \times S^{1}\right) \subset \mathcal{U}\right\} .
$$

Clearly $s_{k}^{\prime}>s_{k}$, thus $s_{k}^{\prime} \rightarrow \infty$. Now define $\widetilde{v}_{k}:\left[-s_{k}^{\prime}, \infty\right) \times S^{1} \rightarrow \mathbb{R} \times M$ by

$$
\tilde{v}_{k}(s, t)=\left(b_{k}(s, t), v_{k}(s, t)\right)=\left(\beta_{k}\left(s+s_{k}^{\prime}, t\right)-\beta_{k}\left(s_{k}^{\prime}, 0\right), w_{k}\left(s+s_{k}^{\prime}, t\right)\right) .
$$

These satisfy a uniform $C^{1}$-bound and are $\widetilde{J}_{k}$-holomorphic with respect to a sequence of complex structures which converge to $i$ in $C_{\mathrm{loc}}^{\infty}\left(\mathbb{R} \times S^{1}\right)$, hence a subsequence converges to a $\widetilde{J}_{\infty}$-holomorphic finite energy cylinder

$$
\tilde{v}_{\infty}=\left(b_{\infty}, v_{\infty}\right): \mathbb{R} \times S^{1} \rightarrow \mathbb{R} \times M .
$$

As in the stable case, the loop $\gamma:=v_{\infty}\left(\{0\} \times S^{1}\right)$ is necessarily nontrivial and not contained in a periodic orbit, thus $\widetilde{v}_{\infty}$ is nonconstant and $E_{\omega_{\infty}}\left(\widetilde{v}_{\infty}\right)>\infty$. The usual topological constraints now imply that both punctures are nonremovable: in particular $\tilde{v}_{\infty}$ is asymptotic to $P_{\infty}$ at $+\infty$, and an $m$-fold covered orbit on $L$ at $-\infty$, with both punctures positive. Denote the $m$-fold covered orbit on $L$ by $P_{-}$.

This leads to the following contradiction. Let $\Psi$ denote the natural trivialization of $\xi_{\infty}$ along $P_{-}$defined by the intersection $T L \cap \xi_{\infty}$. Then if $e_{-}$is the asymptotic eigenfunction at the puncture, we claim wind ${ }^{\Psi}\left(e_{-}\right)=0$. Otherwise, we could find some $s_{0}$ near $-\infty$ such that for large $k$, the loop $u_{k} \circ \varphi_{k} \circ \psi\left(s_{0}, \cdot\right)$ winds nontrivially around $P_{-}$, and must therefore intersect $L$, which is a contradiction. Then Lemma 2.5 gives $\mu_{\mathrm{CZ}}^{\Psi-}\left(P_{-}\right)=1$. In terms of the given trivialization $\Phi_{\infty}$ of $\left.\xi_{\infty}\right|_{M}$, we have $\operatorname{wind}_{P_{-}}^{\Phi_{\infty}}(\Psi)=-m$ and thus $\mu_{\mathrm{CZ}}^{\Phi_{\infty}^{-}}\left(P_{-}\right)=1-2 m$. Now ind $(\widetilde{w})=\mu_{\mathrm{CZ}}(\widetilde{w})=$ $3+1-2 m=4-2 m$, and Theorem 2.4 gives

$$
0 \leq 2 \operatorname{wind}_{\pi}(\widetilde{w}) \leq \operatorname{ind}(\widetilde{w})-2+\# \Gamma_{0}=2-2 m .
$$

This is impossible, since we've assumed $m \geq 2$. We're left with the alternative that $\widetilde{v}^{1}$ is positively asymptotic to $P_{\infty}$ at the marked point $p$.

Before moving on, we should note the following lemma, which was used in the argument above to prove $C^{\infty}$-convergence on the noncompact set $[0, \infty) \times S^{1}$. 
Lemma 3.22 Let $A_{k}: \mathbb{D} \rightarrow \operatorname{End}(T \mathbb{D})$ be a sequence of smooth sections of the tensor bundle $\operatorname{End}(T \mathbb{D}) \rightarrow \mathbb{D}$ such that $A_{k} \rightarrow 0$ in $C^{\infty}(\mathbb{D})$. Then if $\psi:[0, \infty) \times S^{1} \rightarrow \mathbb{D} \backslash\{0\}$ is the biholomorphic map $\psi(s, t)=e^{-2 \pi(s+i t)}$, the tensors $\psi^{*} A_{k}$ on $[0, \infty) \times S^{1}$ converge uniformly to 0 with all derivatives.

Proof Define the Euclidean metric on both $\mathbb{D}$ and $[0, \infty) \times S^{1}$, and use the natural coordinates on each to write sections of $\operatorname{End}(T \mathbb{D})$ or $\operatorname{End}\left(T\left([0, \infty) \times S^{1}\right)\right)$ as smooth real 2-by-2 matrix valued functions. If $\psi(s, t)=z$, then the first derivative of $\psi$ at $(s, t)$ and its inverse can be written as

$$
\begin{gathered}
D \psi(s, t)=-2 \pi e^{-2 \pi(s+i t)}=-2 \pi z, \\
D \psi^{-1}(z)=-\frac{1}{2 \pi z}=-\frac{1}{2 \pi} e^{2 \pi(s+i t)},
\end{gathered}
$$

using the natural inclusion of $\mathbb{C}$ in the space of real 2-by-2 matrices. Then

$$
\left(\psi^{*} A_{k}\right)(s, t)=D \psi^{-1}(z) \circ A_{k}(z) \circ D \psi(s, t)=e^{2 \pi i t} A_{k}(z) e^{-2 \pi i t},
$$

so $\left\|\psi^{*} A_{k}\right\|_{C^{0}}=\left\|A_{k}\right\|_{C^{0}} \rightarrow 0$ since the matrices on either side of $A_{k}(z)$ are orthogonal. We obtain convergence for all derivatives by observing that for any multiindex $\alpha$, $\partial^{\alpha}\left(\psi^{*} A_{k}\right)(s, t)$ is a finite sum of expressions of the form

$$
c \cdot U \cdot e^{2 \pi i t} \cdot D^{j} A_{k}(z)(z, \ldots, z) \cdot e^{-2 \pi i t} \cdot V
$$

where $c$ is a real constant, $U$ and $V$ are constant unitary matrices (ie complex numbers of modulus 1 ), and $j \leq|\alpha|$. This is clearly true for $|\alpha|=0$ and follows easily for all $\alpha$ by induction, using (26). The norm of this expression clearly goes to 0 uniformly in $(s, t)$ as $k \rightarrow \infty$.

Step 3: Degeneration of $j_{k}$ Most of the hard work for this step was done in the proof of Proposition 3.21; in particular, the discussion surrounding Figure 6 and Figure 7 applies in the present situation as well. The main difference here is that, since there are now orbits that are unlinked with $P_{\infty}$, it is not so trivial to exclude interior double points. Unpaired nodes, of course, will not be excluded at all; they will replace the boundary.

\section{Claim $\Delta \cap S_{1}$ is empty.}

This will follow from similar algebraic relations to the ones that were previously used only to exclude boundary double points. At any component $\alpha_{j} \subset \partial S_{1}$, the homotopy class of $v^{1}\left(\alpha_{j}\right)$ in $L_{f(j)}$ is fully determined by (21), giving the Maslov index $-2 \widehat{m}_{j}$ with respect to the given trivialization $\Phi_{\infty}$ of $\left.\xi_{\infty}\right|_{M}$. 
The behavior at an unpaired node $w_{j} \in N \cap S_{1}$ is similarly constrained: by (23), the asymptotic limit $P_{j}$ can only be one of the Morse-Bott orbits on some torus $L_{i}$. Then (22) tells us the torus in question must be $L_{g(j)}$, and since $\operatorname{lk}\left(P_{j}, K_{g(j)}\right)=-1$, the covering number $n_{j}=1$. So $w_{j}$ is a positive puncture, and repeating the argument from Step 2, the asymptotic eigenfunction has zero winding relative to the natural framing determined by $T L_{g(j)} \cap \xi_{\infty}$. Lemma 2.5 then gives Conley-Zehnder index 1 with respect to this framing. The framing itself has winding number -1 along $P_{j}$ with respect to the trivialization $\Phi_{\infty}$, which changes the Conley-Zehnder index to $-2+1=-1$.

Likewise at an interior double point $z_{j} \in \Delta \cap$ int $S_{1}$, the asymptotic limit $P_{j}^{\prime}$ must belong to a Morse-Bott torus, and summing (24) over all components $K_{i} \subset K$ we have

$$
-n_{j}^{\prime}=n_{j}^{\prime} \operatorname{lk}\left(P_{j}^{\prime}, K\right)=\operatorname{lk}\left(u_{k}\left(\partial_{j} \Sigma\right), K\right)=-m_{j},
$$

so $z_{j}$ is a positive puncture with covering number $m_{j}$. The Conley-Zehnder index with respect to the natural framing on the torus is again 1, but now the framing winds $-m_{j}$ times with respect to $\Phi_{\infty}$, giving index $-2 m_{j}+1$.

We now compute the Maslov index

$$
\begin{aligned}
\mu\left(\widetilde{v}^{1}\right) & =3+\ell(-1)+\sum_{j=1}^{q}\left(1-2 m_{j}\right)-2 \sum_{j=1}^{s} \widehat{m}_{j} \\
& =3-\ell+q-2\left(\sum_{j=1}^{q} m_{j}+\sum_{j=1}^{s} \widehat{m}_{j}\right),
\end{aligned}
$$

and (9) gives

$$
\begin{aligned}
\operatorname{ind}\left(\widetilde{v}^{1}\right) & =\mu\left(\widetilde{v}^{1}\right)-\chi\left(\dot{S}_{1}\right)+s \\
& =3-\ell+q-2\left(\sum_{j=1}^{q} m_{j}+\sum_{j=1}^{s} \widehat{m}_{j}\right)-(1-s-\ell-q)+s \\
& =2+2 \sum_{j=1}^{q}\left(1-m_{j}\right)+2 \sum_{j=1}^{s}\left(1-\widehat{m}_{j}\right) .
\end{aligned}
$$

We can assume that at least one of the sets $\partial S_{1}, N \cap S_{1}$ and $\Delta \cap$ int $S_{1}$ is nonempty, in which case $v^{1}$ approaches one of the tori $L_{i}$ somewhere, while approaching $P_{\infty}$ at the marked point $p$. It follows that the image of $v^{1}$ is not contained in any single 
periodic orbit, so $E_{\omega_{\infty}}\left(\widetilde{v}^{1}\right)>0$. Thus Theorem 2.4 gives

$$
0 \leq 2 \operatorname{wind}_{\pi}\left(\widetilde{v}^{1}\right) \leq 2\left(\sum_{j=1}^{q}\left(1-m_{j}\right)+\sum_{j=1}^{s}\left(1-\widehat{m}_{j}\right)\right) .
$$

Recalling that always $m_{j} \geq 2$ and $\widehat{m}_{j} \geq 1$, we conclude $q=0$ and $\widehat{m}_{j}=1$ for each $j$, so $\Delta \cap$ int $S_{1}$ is empty, and by the same argument as in the proof of Theorem 3.4, so is $\Delta \cap \partial S_{1}$.

Claim $\partial S_{1}=\varnothing$ and $\# N=m$.

We've now established that $S$ can have only one connected component (there are no double points to connect $S_{1}$ with anything else), thus $\overline{\mathbf{S}}_{r}=\bar{S}_{1} \cong \Sigma$, and $m=s+\ell$. We need to prove $s=0$. Having just shown that everything on the right hand side of (27) vanishes, we have $\operatorname{wind}_{\pi}\left(\widetilde{v}^{1}\right)=0$, so $v: \dot{S}_{1} \rightarrow S^{3}$ is immersed and transverse to $X_{\infty}$. But if $\partial S_{1} \neq \varnothing$ this cannot be true, because $v^{1}\left(\partial S_{1}\right)$ and all orbits of $X_{\infty}$ on $L_{j}$ are meridians.

By the above results, $S$ is a sphere with one marked point $p$ and unpaired nodes $N=\left\{w_{1}, \ldots, w_{m}\right\} \subset S \backslash\{p\}$, so we can identify it holomorphically with the Riemann sphere $\left(S^{2}, i\right)$, setting $\infty:=p$ and $\Gamma^{\prime}:=N$. The diffeomorphisms $\varphi_{k}: \overline{\mathbf{S}}_{r} \rightarrow \Sigma$ preserve $\infty$, and restricting them to the interior they define diffeomorphisms

$$
\varphi_{k}: S \backslash \Gamma^{\prime} \rightarrow \text { int } \Sigma,
$$

with $\varphi_{k}^{*} j_{k} \rightarrow i$ in $C_{\text {loc }}^{\infty}\left(S \backslash \Gamma^{\prime}\right)$. Moreover, after $\mathbb{R}$-translation, $\tilde{u}_{k} \circ \varphi_{k} \rightarrow \widetilde{v}^{1}$ in $C_{\text {loc }}^{\infty}\left(S \backslash\left(\{\infty\} \cup \Gamma^{\prime}\right), \mathbb{R} \times S^{3}\right)$, and $\widetilde{v}^{1}$ has precisely the required asymptotic behavior at the punctures $\infty$ and $w_{j} \in \Gamma^{\prime}$. This concludes the proof of $C_{\mathrm{loc}}^{\infty}$-convergence for Theorem 3.5.

\subsection{Convergence at the punctures}

To finish proving Theorem 3.4 and Theorem 3.5, it remains only to establish that the sequences of maps $\left(a_{k}+c_{k}, u_{k}\right) \circ \varphi_{k}$ behave well on small neighborhoods of the punctures and boundary. This follows from the next three results.

Lemma 3.23 Let $j_{k}$ be a sequence of complex structures on $\dot{\mathbb{D}}:=\mathbb{D} \backslash\{0\}$ such that $j_{k} \rightarrow i$ in $C_{\mathrm{loc}}^{\infty}(\dot{\mathbb{D}})$, and take a sequence of biholomorphic maps

$$
\psi_{k}:\left(\left[0, R_{k}\right) \times S^{1}, i\right) \rightarrow\left(\dot{\mathbb{D}}, j_{k}\right)
$$

for $R_{k} \in(0, \infty]$. Then after passing to a subsequence, $R_{k} \rightarrow \infty$ and $\psi_{k}$ converges in $C_{\mathrm{loc}}^{\infty}\left([0, \infty) \times S^{1}, \dot{\mathbb{D}}\right)$ to a biholomorphic map $\psi:\left([0, \infty) \times S^{1}, i\right) \rightarrow(\dot{\mathbb{D}}, i)$. 
This can be proved by a routine bubbling off analysis for the embedded holomorphic maps $\psi_{k}^{-1}:\left(\dot{\mathbb{D}}, j_{k}\right) \hookrightarrow\left(\mathbb{R} \times S^{1}, i\right)$; we refer to [29] for the details. With this preparation, we can reduce the problem of convergence at the boundary and ends to the following two statements; we'll prove only the second, since both use almost identical arguments. Let $\mathcal{H}_{k}=\left(\xi_{k}, X_{k}, \omega_{k}, J_{k}\right)$ be a sequence of stable Hamiltonian structures on a compact 3-manifold $M$ with boundary, converging in $C^{\infty}$ to $\mathcal{H}_{\infty}=\left(\xi_{\infty}, X_{\infty}, \omega_{\infty}, J_{\infty}\right)$, with associated almost complex structures $\widetilde{J}_{k} \rightarrow \widetilde{J}_{\infty}$. Assume also that the taming forms $\omega_{k}$ are exact.

Proposition 3.24 Assume $P \subset M$ is a nondegenerate periodic orbit of $X_{k}$ for all $k \leq \infty$. Suppose $\widetilde{v}_{k}=\left(b_{k}, v_{k}\right):[0, \infty) \times S^{1} \rightarrow \mathbb{R} \times M$ is a sequence of finite energy $\widetilde{J}_{k}$-holomorphic maps asymptotic to $P$, with uniformly bounded energy $E_{k}\left(\widetilde{v}_{k}\right)<$ $C$ and converging in $C_{\mathrm{loc}}^{\infty}\left([0, \infty) \times S^{1}, \mathbb{R} \times M\right)$ to a $\widetilde{J}_{\infty}$-holomorphic map $\widetilde{v}_{\infty}=$ $\left(b_{\infty}, v_{\infty}\right):[0, \infty) \times S^{1} \rightarrow \mathbb{R} \times M$, also asymptotic to $P$. Then for every sequence $s_{k} \rightarrow \infty$, the loops $v_{k}\left(s_{k}, \cdot\right)$ converge in $C^{\infty}\left(S^{1}, M\right)$ to a parameterization of $P$.

Proposition 3.25 Assume $L \subset M$ is a 2-torus which is tangent to all $X_{k}$ and is a Morse-Bott torus for $X_{\infty}$. Let $R_{k} \rightarrow \infty$, and suppose $\tilde{v}_{k}=\left(b_{k}, v_{k}\right):\left[0, R_{k}\right] \times S^{1} \rightarrow$ $\mathbb{R} \times M$ is a sequence of $\widetilde{J}_{k}$-holomorphic maps converging in $C_{\mathrm{loc}}^{\infty}\left([0, \infty) \times S^{1}, \mathbb{R} \times M\right)$ to a $\widetilde{J}_{\infty}$-holomorphic half-cylinder $\widetilde{v}_{\infty}=\left(b_{\infty}, v_{\infty}\right):[0, \infty) \times S^{1} \rightarrow \mathbb{R} \times M$, and satisfying a uniform energy bound $E_{k}\left(\widetilde{v}_{k}\right)<C$. Assume also that $\widetilde{v}_{k}\left(\left\{R_{k}\right\} \times S^{1}\right) \subset$ $\left\{c_{k}\right\} \times L$ for some sequence $c_{k} \in \mathbb{R}$, and $\widetilde{v}_{\infty}$ is asymptotic to a periodic orbit $P \subset L$ which is homotopic along $L$ to each of the loops $v_{k}\left(\left\{R_{k}\right\} \times S^{1}\right)$. Then for every sequence $s_{k} \in\left[0, R_{k}\right]$ with $s_{k} \rightarrow \infty$, the loops $v_{k}\left(s_{k}, \cdot\right)$ have a subsequence convergent in $C^{\infty}\left(S^{1}, M\right)$ to a closed orbit homotopic to $P$ in $L$.

Proof We claim first that for any sequence $s_{k} \leq R_{k}$ with $s_{k} \rightarrow \infty$,

$$
\int_{\left[s_{k}, R_{k}\right] \times S^{1}} v_{k}^{*} \omega_{k} \rightarrow 0
$$

Indeed, the loop $v_{k}\left(s_{0}, \cdot\right)$ can be made arbitrarily close in $C^{\infty}\left(S^{1}, M\right)$ to a parameterization of $P$ by choosing $s_{0}$ and $k$ large enough. Then for any $\epsilon>0$, the exactness of $\omega_{k}$ implies that we can find $k_{0} \in \mathbb{N}$ and $s_{0} \in\left[0, R_{k_{0}}\right]$ such that

$$
\int_{\left[s_{0}, R_{k}\right] \times S^{1}} v_{k}^{*} \omega_{k}<\epsilon
$$

for all $k \geq k_{0}$. Since $v_{k}^{*} \omega_{k}$ is positive, $\int_{\left[s_{k}, R_{k}\right] \times S^{1}} v_{k}^{*} \omega_{k}$ is bounded by this as soon as $s_{k} \geq s_{0}$, proving the claim. 
From this and the uniform energy bound, we use the same argument as in Theorem 3.12 to derive a uniform bound on $\left|d \widetilde{v}_{k}\right|$ : else a nonconstant finite energy plane or half-plane with zero $\omega_{\infty}$-energy bubbles off, contradicting Proposition 3.11.

Consider now a sequence $s_{k} \leq R_{k}$ with $s_{k} \rightarrow \infty$, and suppose $R_{k}-s_{k}$ is unbounded. Then a subsequence of

$$
\widetilde{w}_{k}:\left[-s_{k}, R_{k}-s_{k}\right] \times S^{1} \rightarrow \mathbb{R} \times M:(s, t) \mapsto \widetilde{v}_{k}\left(s+s_{k}, t\right)
$$

converges (after $\mathbb{R}$-translation) in $C_{\text {loc }}^{\infty}\left(\mathbb{R} \times S^{1}, \mathbb{R} \times M\right)$ to a $\widetilde{J}_{\infty}$-holomorphic finite energy cylinder $\tilde{w}_{\infty}=\left(\beta_{\infty}, w_{\infty}\right): \mathbb{R} \times S^{1} \rightarrow \mathbb{R} \times M$ with $E_{\omega_{\infty}}\left(\tilde{w}_{\infty}\right)=0$. By Proposition 3.11, such an object is either constant or an orbit cylinder. To rule out the former, we claim

$$
\int_{\{0\} \times S^{1}} w_{\infty}^{*} \lambda_{\infty}=\lim \int_{\left\{s_{k}\right\} \times S^{1}} v_{k}^{*} \lambda_{k}=\int_{P} \lambda_{\infty}=: Q_{0} \neq 0 .
$$

Indeed, since $v_{k}\left(R_{k}, \cdot\right)$ is homotopic to $P$ and $d \lambda_{k}$ vanishes along $L$, we can assume for $k$ sufficiently large that

$$
\left|Q_{0}-\int_{\left\{R_{k}\right\} \times S^{1}} v_{k}^{*} \lambda_{k}\right|<\epsilon .
$$

Then assuming also $\int_{\left[s_{k}, R_{k}\right] \times S^{1}} v_{k}^{*} \omega_{k}<\epsilon$ and using Lemma 3.15,

$$
\begin{aligned}
\left|Q_{0}-\int_{\left\{s_{k}\right\} \times S^{1}} v_{k}^{*} \lambda_{k}\right| & =\left|Q_{0}-\int_{\left\{R_{k}\right\} \times S^{1}} v_{k}^{*} \lambda_{k}+\int_{\left[s_{k}, R_{k}\right] \times S^{1}} v_{k}^{*} d \lambda_{k}\right| \\
& \leq(1+C) \epsilon .
\end{aligned}
$$

Consequently, $\widetilde{w}_{\infty}$ is a trivial cylinder over a closed orbit with the same period as $P$.

If instead $R_{k}-s_{k}$ remains bounded as $k \rightarrow \infty$, we consider the maps

$$
\widetilde{w}_{k}:\left[-R_{k}, 0\right] \times S^{1} \rightarrow \mathbb{R} \times M:(s, t) \mapsto \widetilde{v}_{k}\left(s+R_{k}, t\right),
$$

which satisfy the boundary condition $\widetilde{w}_{k}\left(\{0\} \times S^{1}\right) \subset\left\{c_{k}\right\} \times L$. Then a subsequence converges in $C_{\text {loc }}^{\infty}\left((-\infty, 0] \times S^{1}, \mathbb{R} \times M\right)$ after $\mathbb{R}$-translation to a $\widetilde{J}_{\infty}$-holomorphic finite energy half-cylinder $\widetilde{w}_{\infty}:(-\infty, 0] \times S^{1} \rightarrow \mathbb{R} \times M$, with $E_{\omega_{\infty}}\left(\widetilde{w}_{\infty}\right)=0$. Repeating the argument above, $\widetilde{w}_{\infty}$ parametrizes half of an orbit cylinder.

We've shown now that for every sequence $s_{k} \rightarrow \infty$ with $s_{k} \leq R_{k}$, the sequence of loops $v_{k}\left(s_{k}, \cdot\right)$ has a subsequence converging in $C^{\infty}\left(S^{1}, M\right)$ to a closed orbit of $X_{\infty}$. We claim finally that this orbit lies in $L$. If not, then we can find a sequence $s_{k}^{\prime} \in\left(s_{k}, R_{k}\right)$ such that the loops $v_{k}\left(s_{k}^{\prime}, \cdot\right)$ touch the boundary of a small neighborhood of $L$. But by the Morse-Bott condition, we may assume this neighborhood contains 
no other closed orbits of the same period, thus $s_{k}^{\prime}$ can have no subsequence for which $v_{k}\left(s_{k}^{\prime}, \cdot\right)$ converges to an appropriate orbit, giving a contradiction.

\section{The main construction}

\subsection{Surgery and Lutz twists on transverse links}

We now define precisely the type of surgery on contact manifolds that we wish to perform. In the following, $S^{1}$ is always defined to be the quotient $\mathbb{R} / \mathbb{Z}$.

Lemma 4.1 Let $(\theta, \rho, \phi)$ be the standard cylindrical polar coordinates on $S^{1} \times \mathbb{R}^{2}$, oriented by the basis $\left(\partial_{\theta}, \partial_{\rho}, \partial_{\phi}\right)$. For $\rho \geq 0$, choose real-valued functions $f(\rho)$ and $g(\rho)$ such that $(\rho, \phi) \mapsto f(\rho)$ and $(\rho, \phi) \mapsto g(\rho) / \rho^{2}$ define smooth functions on $\mathbb{R}^{2}$. Then

$$
\lambda:=f(\rho) d \theta+g(\rho) d \phi
$$

defines a smooth $1-$ form on $S^{1} \times \mathbb{R}^{2}$, which is a positive contact form if and only if the following two conditions are met:

(i) the Wronskian $D(\rho):=f(\rho) g^{\prime}(\rho)-f^{\prime}(\rho) g(\rho)>0$ for all $\rho>0$,

(ii) $f(0) g^{\prime \prime}(0)>0$.

In that case, the corresponding Reeb vector field is given by

$$
X(\theta, \rho, \phi)=\frac{1}{D(\rho)}\left(g^{\prime}(\rho) \partial_{\theta}-f^{\prime}(\rho) \partial_{\phi}\right) .
$$

Proof A simple calculation shows that

$$
\lambda \wedge d \lambda=D(\rho) d \theta \wedge d \rho \wedge d \phi=\frac{D(\rho)}{\rho} d \theta \wedge d x \wedge d y,
$$

where $(x, y)$ are Cartesian coordinates on $\mathbb{R}^{2}$. Then $\lim _{\rho \rightarrow 0} D(\rho) / \rho=D^{\prime}(0)=$ $f(0) g^{\prime \prime}(0)$, and it is straightforward to verify that the expression for $X$ above satisfies $d \lambda(X, \cdot) \equiv 0$ and $\lambda(X) \equiv 1$.

Intuitively, these conditions on $f$ and $g$ mean that the curve $\rho \mapsto(f(\rho), g(\rho))$ always winds counterclockwise around the origin in the $x y$-plane, beginning on the $x$-axis with zero velocity and nonzero angular acceleration.

Let $(M, \xi)$ be an oriented 3-manifold with a positive and cooriented contact structure, and suppose $K \subset M$ is an oriented knot which is positively transverse to $\xi$, ie its 
orientation matches the coorientation of $\xi$. A Lutz twist along $K$ is defined as follows. By the contact neighborhood theorem, $K$ has a solid torus neighborhood $N_{K} \subset M$ which can be identified with $S^{1} \times \overline{B_{\epsilon}^{2}(0)}$, where $\overline{B_{\epsilon}^{2}(0)}$ is the closed ball of radius $\epsilon$ around the origin in $\mathbb{R}^{2}$, such that $K=S^{1} \times\{0\}$ and $\left.\xi\right|_{N_{K}}$ is the kernel of

$$
\lambda_{0}:=d \theta+\rho^{2} d \phi,
$$

using the cylindrical polar coordinates of Lemma 4.1. We then change $\xi$ by replacing $\lambda_{0}$ on $N_{K}$ with $\lambda_{K}:=f(\rho) d \theta+g(\rho) d \phi$, where $f$ and $g$ are functions chosen as in Lemma 4.1 so that $\lambda_{K}$ is a contact form, and furthermore the following hold.

(1) There exists $\delta \in(0, \epsilon)$ such that $(f(\rho), g(\rho))=\left(1, \rho^{2}\right)$ for $\rho \geq \delta$.

(2) The trajectory $\rho \mapsto(f(\rho), g(\rho))$ rotates at least halfway around the origin for $\rho \in[0, \delta]$ (see Figure 9 and Figure 10).

This operation produces a new contact structure $\xi_{K}=\operatorname{ker} \lambda_{K}$, such that there exists at least one radius $\rho_{0} \in(0, \delta)$ at which $g\left(\rho_{0}\right)=0$. This means the meridian $\left\{\left(0, \rho_{0}, \phi\right) \mid \phi \in \mathbb{R} / 2 \pi \mathbb{Z}\right\}$ is Legendrian and forms the boundary of an overtwisted disk, so that $\xi_{K}$ is necessarily overtwisted. Relatedly, there is at least one radius $\rho_{1} \in\left(0, \rho_{0}\right)$ at which $g^{\prime}\left(\rho_{1}\right)=0$ and $f^{\prime}\left(\rho_{1}\right)>0$, so that the Reeb vector field on the torus $\left\{\rho=\rho_{1}\right\}$ generates periodic orbits which are negatively oriented meridians. This detail will be important for constructing finite energy foliations in such neighborhoods.

The twists shown in Figure 9 and Figure 10 may be called the "half Lutz twist" and "full Lutz twist" respectively: the former changes the homotopy class of $\xi$ as a 2-plane distribution, while the latter does not (see Bennequin [2] for an explicit homotopy). The half Lutz twist is particularly important for the following reason: by an obstruction theory argument due originally to Lutz [20;21], any homotopy class of cooriented 2-plane distributions on $M$ admits a positive contact structure, which can be obtained from any other $\xi$ by half Lutz twists along some positively transverse link. See Geiges [7] for a fuller discussion of this result.

We next generalize this to a twisting version of nontrivial Dehn surgery on contact manifolds. Assume $M=S^{3}$ with positive contact structure $\xi$ and positively transverse knot $K \subset S^{3}$. Identify a neighborhood $N_{K}$ of $K$ once more with $S^{1} \times B_{\epsilon}^{2}(0)$, requiring in particular that $\left.\xi\right|_{N_{K}}$ be the kernel of $\lambda_{0}=d \theta+\rho^{2} d \phi$ and that the longitude $\left\{(\theta, \epsilon, 0) \mid \theta \in S^{1}\right\} \subset S^{3}$ be homologous to zero in $S^{3} \backslash K$. Let $\lambda_{1}=$ $f_{1}(\rho) d \theta+g_{1}(\rho) d \phi$ be a contact form on $N_{K}$ obtained from $\lambda_{0}$ by a Lutz twist as described above, choosing $f_{1}$ and $g_{1}$ so that there is a radius $\rho_{1} \in(0, \epsilon)$ at which $g_{1}^{\prime}\left(\rho_{1}\right)=0$ and $f_{1}^{\prime}\left(\rho_{1}\right)>0$, while $g_{1}^{\prime}(\rho)>0$ for all $\rho \in\left(\rho_{1}, \epsilon\right]$. 
A framing of $K$ is a number $p / q \in \mathbb{Q} \cup\{\infty\}$, where we assume $p$ and $q$ are relatively prime integers. Define now another solid torus $N^{\prime}:=S^{1} \times \overline{B_{\epsilon}^{2}(0)}$, with canonical cylindrical polar coordinates $\left(\theta^{\prime}, \rho^{\prime}, \phi^{\prime}\right)$. Define also $\eta:=\phi / 2 \pi, \eta^{\prime}:=\phi^{\prime} / 2 \pi \in S^{1}$, so that the pairs $(\theta, \eta),\left(\theta^{\prime}, \eta^{\prime}\right) \in S^{1} \times S^{1}$ give coordinates on the tori $\partial N_{K}$ and $\partial N^{\prime}$ respectively. Now choose $\delta \in\left(0, \rho_{1}\right)$ and define an embedding

$$
\psi: N^{\prime} \backslash\left(S^{1} \times B_{\delta}^{2}(0)\right) \hookrightarrow N_{K}:\left(\theta^{\prime}, \rho^{\prime}, \phi^{\prime}\right) \mapsto\left(\theta\left(\theta^{\prime}, \phi^{\prime}\right), \rho^{\prime}, \phi\left(\theta^{\prime}, \phi^{\prime}\right)\right),
$$

where the map $\left(\theta^{\prime}, \phi^{\prime}\right) \mapsto(\theta, \phi)$ is determined by an orientation preserving diffeomorphism $\partial N^{\prime} \mapsto \partial N_{K}$ of the form

$$
\left(\begin{array}{l}
\theta \\
\eta
\end{array}\right)=\left(\begin{array}{ll}
n & q \\
m & p
\end{array}\right)\left(\begin{array}{l}
\theta^{\prime} \\
\eta^{\prime}
\end{array}\right)
$$

for some matrix in $\operatorname{SL}(2, \mathbb{Z})$. A new manifold $M_{K}$ is defined by removing $S^{1} \times$ $B_{\delta}^{2}(0) \subset N_{K}$ from $S^{3}$ and gluing in $N^{\prime}$ via this embedding. The topological type of $M_{K}$ depends only on $p / q \in \mathbb{Q} \cup\{\infty\}$ (see Saveliev [24]).

The contact form $\lambda_{1}$ on $N_{K}$ pulls back via $\psi$ to a contact form $\lambda_{K}$ on $N^{\prime} \backslash\left(S^{1} \times\right.$ $\left.B_{\delta}^{2}(0)\right)$, which in the coordinates $\left(\theta^{\prime}, \rho^{\prime}, \phi^{\prime}\right)$ has the form

$$
\begin{aligned}
\lambda_{K} & =f_{K}\left(\rho^{\prime}\right) d \theta^{\prime}+g_{K}\left(\rho^{\prime}\right) d \phi^{\prime} \\
& =\left[n f_{1}\left(\rho^{\prime}\right)+2 \pi m g_{1}\left(\rho^{\prime}\right)\right] d \theta^{\prime}+\left[\frac{q}{2 \pi} f_{1}\left(\rho^{\prime}\right)+p g_{1}\left(\rho^{\prime}\right)\right] d \phi^{\prime} .
\end{aligned}
$$

Clearly $\xi_{K}:=\operatorname{ker} \lambda_{K}$ has a natural extension to $M_{K} \backslash N^{\prime}$, and we can extend $f_{K}$ and $g_{K}$ to $\rho \in[0, \delta]$ so that $\lambda_{K}$ becomes a contact form on $N^{\prime}$.

We will refer to the operation described above as a rational twist surgery along $K$, or integral in the case where $p / q \in \mathbb{Z} \cup\{\infty\}$, ie $q= \pm 1$ or 0 . Observe that when $q=0$, we can choose the surgery matrix to be the identity, which gives simply a Lutz twist along $K$. By the theorem of Lickorish [19] and Wallace [27], every closed oriented 3-manifold $M$ can be obtained by integral surgery along some link $K \subset S^{3}$; then making $K$ positively transverse by a $C^{0}$-perturbation, the procedure we've described proves the result of Martinet [22] that every such 3-manifold admits a cooriented and positive contact structure $\xi$.

Let $N \subset S^{3}$ be the union of all the solid tori removed from $S^{3}$ in the above gluing, and let $N^{\prime} \subset M$ be the corresponding solid tori that are glued in, so there's a natural diffeomorphism $S^{3} \backslash N=M \backslash N^{\prime}$. The Lutz argument now provides a link $K_{1} \subset M$, positively transverse to $\xi_{K}$, such that the contact structure $\xi_{1}$ obtained by half Lutz twists along the components of $K_{1}$ may represent any desired homotopy class. In particular, we can specify the homotopy class of $\xi_{1}$ over the complement of a small 
3-ball $B \subset M$ by choosing any link $K^{\prime}$ representing the appropriate class in $H_{1}(M)$, then perturbing it to be positively transverse-note that we're thus free to assume $K^{\prime} \subset M \backslash N^{\prime}$. We can also assume $B \subset M \backslash N^{\prime}$, and then the homotopy class of $\xi^{\prime}$ over $B$ can be changed as needed by twisting along a transverse link $K^{\prime \prime} \subset B$ with the appropriate self-linking number. In summary, we can assume the transverse link $K_{1}$ needed to change the homotopy class of $\xi_{K}$ lies in $M \backslash N^{\prime}=S^{3} \backslash N$, and this leads to be following statement of the famous Lutz-Martinet theorem.

Theorem 4.2 (Lutz, Martinet) Given a closed oriented 3-manifold $M$ with a cooriented 2-plane distribution $\alpha$, there exists a positive contact structure $\xi$ homotopic to $\alpha$, and $(M, \xi)$ can be obtained from the tight three-sphere $\left(S^{3}, \xi_{0}\right)$ by a rational twist surgery along some transverse link.

Remark 4.3 By Eliashberg's classification theorem for overtwisted contact structures [4], the procedure above produces every overtwisted contact structure on every closed 3-manifold.

The main goal of this paper is to construct finite energy foliations on contact manifolds obtained from $\left(S^{3}, \xi_{0}\right)$ by twist surgeries. The following result will be helpful for establishing that these foliations can be made to have certain nice properties, eg that all punctures are positive and all orbits are simply covered.

Proposition 4.4 For the surgery in Theorem 4.2, we can assume without loss of generality any or all of the following.

(1) The surgery is integral.

(2) $\xi$ is overtwisted.

(3) For each component $K_{j} \subset K$, let $\rho_{1} \in(0, \epsilon)$ be the largest radius where $g_{1}^{\prime}\left(\rho_{1}\right)=$ 0 . Then for all $\rho \in\left(0, \rho_{1}\right]$,

$$
f_{K}^{\prime}(\rho) g_{K}^{\prime \prime}(\rho)-f_{K}^{\prime \prime}(\rho) g_{K}^{\prime}(\rho)>0 .
$$

(4) If the surgery at $K_{j}$ is topologically nontrivial (ie $q \neq 0$ ), $\rho_{1}$ is the radius above and $r \in(0, \epsilon)$ is the smallest radius at which $f_{K}^{\prime}(r) / g_{K}^{\prime}(r)=f_{K}^{\prime}\left(\rho_{1}\right) / g_{K}^{\prime}\left(\rho_{1}\right)$, then

$$
\frac{f_{K}^{\prime}(r)}{g_{K}^{\prime}(r)}-\frac{f_{K}^{\prime \prime}(0)}{g_{K}^{\prime \prime}(0)}
$$

is positive and close to 0 . 
Proof The Lickorish-Wallace theorem guarantees that integral surgeries are sufficient. If the surgery is topologically nontrivial (ie not merely a Lutz twist) at each component of $K$, then the contact structure $\xi$ defined as above need not generally be overtwisted. We can, however, make it overtwisted by performing an extra full Lutz twist along a transverse knot disjoint from $K$; this doesn't change the homotopy class of $\xi$. Condition (3) simply means that the trajectory $\rho \mapsto(f(\rho), g(\rho))$ in $\mathbb{R}^{2}$ has nonzero inward acceleration for all $\rho \in\left(0, \rho_{1}\right]$. Condition (4) is a relation between the slopes of the trajectory at $\rho=0$ and $\rho=r$, and can always be achieved by changing $f_{K}$ and $g_{K}$ near $\rho=0$. This change may involve an extra half Lutz twist, which changes the homotopy class of $\xi$, but it can be changed back by adding Lutz twists along extra transverse knots.

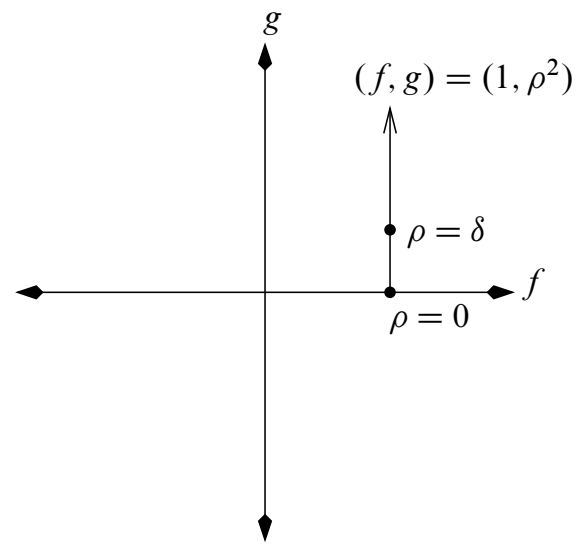

Figure 8: The trajectory $\rho \mapsto(f(\rho), g(\rho))$ for the contact structure $\lambda_{0}=$ $f(\rho) d \theta+g(\rho) d \phi=d \theta+\rho^{2} d \phi$.

It will be helpful to know that transverse links in the tight 3 -sphere $\left(S^{3}, \xi_{0}\right)$ can be assumed after transverse isotopy to approximate covers of Hopf circles, as the latter admit coordinate neighborhoods in which the standard contact form takes an especially simple form. In the following, we view $S^{3}$ as the unit sphere in $\mathbb{C}^{2}$, with

$$
\lambda_{0}(z) v:=\frac{1}{2}\langle i z, v\rangle,
$$

for $z \in S^{3} \subset \mathbb{C}^{2}$ and $v \in T_{z} S^{3} \subset \mathbb{C}^{2}$, where $\langle$,$\rangle is the standard Euclidean inner$ product. It was shown by Bennequin [2] that all transverse links in the standard contact $\mathbb{R}^{3}$ are transversely isotopic to closed braids about the $z$-axis. Using a contact embedding of $\mathbb{R}^{3}$ into the tight 3 -sphere, one can then prove the following lemma (see [29] for details). 


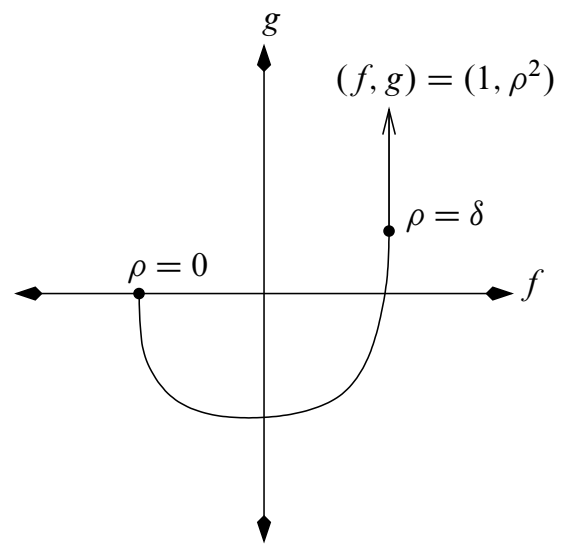

Figure 9: Half Lutz twist of $\lambda_{0}$.

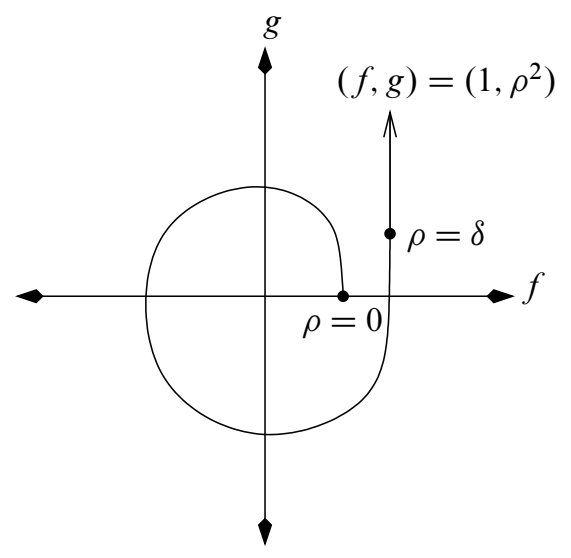

Figure 10: Full Lutz twist of $\lambda_{0}$.

Lemma 4.5 Let $K \subset S^{3}$ be a link positively transverse to the standard contact structure $\xi_{0}$. Then for each component $K_{j} \subset K$ there is a smooth immersion $F_{j}:[0,1] \times S^{1} \rightarrow S^{3}$ such that $F_{j}(1, \cdot): S^{1} \rightarrow S^{3}$ parametrizes $K_{j}, F_{j}(0, t)=\left(e^{2 \pi i k_{j} t}, 0\right)$ for some $k_{j} \in \mathbb{N}$, and for all fixed $\tau \in(0,1]$, the collection of maps $F_{j}(\tau, \cdot): S^{1} \rightarrow S^{3}$ parametrizes a transverse link.

\subsection{Some simple foliations in $S^{1} \times \mathbb{R}^{2}$}

In this section we construct stable finite energy foliations on the local neighborhoods that arise from twist surgery on transverse links. Let $M=S^{1} \times \mathbb{R}^{2}$, with cylindrical polar coordinates $(\theta, \rho, \phi)$ as in the previous section. Then using Lemma 4.1, define a 
positive contact form

$$
\lambda=f(\rho) d \theta+g(\rho) d \phi,
$$

where

$$
D(\rho):=f(\rho) g^{\prime}(\rho)-f^{\prime}(\rho) g(\rho)>0 \text { for all } \rho>0 \text {, and } f(0) g^{\prime \prime}(0)>0 .
$$

Notice that smoothness at $\rho=0$ requires $g(0)=f^{\prime}(0)=g^{\prime}(0)=0$.

The contact structure $\xi=\operatorname{ker} \lambda$ is spanned for all $\rho>0$ by the two vector fields

$$
v_{1}(\theta, \rho, \phi)=\partial_{\rho}, \quad v_{2}(\theta, \rho, \phi)=\frac{1}{D(\rho)}\left(-g(\rho) \partial_{\theta}+f(\rho) \partial_{\phi}\right),
$$

and the Reeb vector field $X$ is given by (28). The flow of $X$ and its linearization are quite easy to compute, leading to the following characterization of periodic orbits.

Proposition 4.6 Suppose $r>0$ and $f^{\prime}(r) / 2 \pi g^{\prime}(r)=p / q \in \mathbb{Q} \cup\{\infty\}$ for relatively prime integers $p$ and $q$, whose signs match the signs of $f^{\prime}(r)$ and $g^{\prime}(r)$ respectively. Then the torus

$$
L_{r}:=\{\rho=r\} \subset M
$$

is foliated by closed orbits of the form

$$
x(t)=\left(\theta_{0}+\frac{g^{\prime}(r)}{D(r)} t, r, \phi_{0}-\frac{f^{\prime}(r)}{D(r)} t\right)=\left(\theta_{0}+\frac{q}{T} t, r, \phi_{0}-\frac{2 \pi p}{T} t\right),
$$

all having minimal period

$$
T=q \frac{D(r)}{g^{\prime}(r)}=2 \pi p \frac{D(r)}{f^{\prime}(r)}
$$

(in the cases where $f^{\prime}(r)=p=0$ or $g^{\prime}(r)=q=0$, pick whichever one of these expressions makes sense). The torus is Morse-Bott if and only if the function $\rho \mapsto$ $f^{\prime}(\rho) / g^{\prime}(\rho)$ (or its reciprocal) has nonvanishing derivative at $r$.

Likewise, the circle

$$
P:=\{\rho=0\} \subset M
$$

is a closed orbit with minimal period $T=|f(0)|$. For $k \in \mathbb{N}$, its $k$-fold cover $P^{k}$ is degenerate if and only if $\frac{k f^{\prime \prime}(0)}{2 \pi g^{\prime \prime}(0)} \in \mathbb{Z}$, and otherwise has

$$
\mu_{C Z}^{\Phi_{0}}\left(P^{k}\right)=2\left\lfloor-\frac{k f^{\prime \prime}(0)}{2 \pi g^{\prime \prime}(0)}\right\rfloor+1,
$$

where $\Phi_{0}$ is the natural symplectic trivialization of $\xi$ along $P$ induced by the coordinates. Here $\lfloor x\rfloor$ means the greatest integer $\leq x$. 
In terms of the curve $\rho \mapsto(f(\rho), g(\rho))$ in $\mathbb{R}^{2}$, this says that the torus $L_{r}$ is Morse-Bott when the slope of the curve has nonvanishing derivative at $r$. The nondegeneracy and index of any cover of $P$ depend similarly on the slope of this curve as it pushes off from the $x$-axis at $\rho=0$.

We've chosen the vector fields $v_{1}$ and $v_{2}$ above so that $d \lambda\left(v_{1}, v_{2}\right) \equiv 1$, ie they give a symplectic trivialization of $\xi$ over $M \backslash P$. Use these now to define an admissible complex multiplication $J$ by

$$
J v_{1}=\beta(\rho) v_{2}, \quad J v_{2}=-\frac{1}{\beta(\rho)} v_{1}
$$

for some smooth function $\beta(\rho)$. The behavior of $\beta$ near 0 can be chosen to ensure that $J$ is smooth at $\rho=0$. Then an $\mathbb{R}$-invariant almost complex structure $\tilde{J}$ on $\mathbb{R} \times M$ is defined in the standard way, and we seek maps $\tilde{u}:(S, j) \rightarrow(\mathbb{R} \times M, \widetilde{J})$ defined on a Riemann surface $(S, j)$ and satisfying $T \tilde{u} \circ j=\widetilde{J} \circ T \tilde{u}$. Choosing conformal coordinates $(s, t)$ on $S$, the map $u$ can be written in coordinates as $u(s, t)=$ $(\theta(s, t), \rho(s, t), \phi(s, t))$, and then the Cauchy-Riemann equation becomes

$$
\begin{array}{ll}
a_{s}=f \theta_{t}+g \phi_{t} & \rho_{s}=\frac{1}{\beta}\left(f^{\prime} \theta_{t}+g^{\prime} \phi_{t}\right) \\
a_{t}=-f \theta_{s}-g \phi_{s} & \rho_{t}=-\frac{1}{\beta}\left(f^{\prime} \theta_{s}+g^{\prime} \phi_{s}\right) .
\end{array}
$$

Given two concentric tori $L_{ \pm}=\left\{\rho=\rho_{ \pm}\right\}$, each foliated by periodic orbits that are homologous (up to a sign) in $H_{1}(M \backslash P)$, we shall now construct a stable finite energy foliation of the region between them, each leaf being a cylinder with ends asymptotic to orbits at $L_{-}$and $L_{+}$respectively (Figure 11).

In particular, suppose there are two radii $\rho_{ \pm}$with $0<\rho_{-}<\rho_{+}$, such that

$$
\frac{f^{\prime}\left(\rho_{ \pm}\right)}{2 \pi g^{\prime}\left(\rho_{ \pm}\right)}=\frac{p}{q} \in \mathbb{Q} \cup\{\infty\} \quad \text { and } \quad \frac{f^{\prime}(\rho)}{2 \pi g^{\prime}(\rho)} \neq \frac{p}{q} \text { for } \rho \in\left(\rho_{-}, \rho_{+}\right)
$$

A choice of sign must be made for $p$ and $q$ : for reasons that will become clear shortly, let us choose both so that the quantity $q f^{\prime}(\rho)-2 \pi p g^{\prime}(\rho)$ is positive for $\rho \in\left(\rho_{-}, \rho_{+}\right)$. The two tori $L_{ \pm}$are each foliated by families of periodic orbits, of the form

$$
x_{ \pm}(t)=\left(\theta_{0}+\frac{q_{ \pm}}{T_{ \pm}} t, \rho_{ \pm}, \phi_{0}-\frac{2 \pi p_{ \pm}}{T_{ \pm}} t\right) .
$$

Here $p_{ \pm}$and $q_{ \pm}$are the same as $p$ and $q$ up to a sign, which must be chosen so that the periods $T_{ \pm}=\frac{q_{ \pm} D\left(\rho_{ \pm}\right)}{g^{\prime}\left(\rho_{ \pm}\right)}=\frac{2 \pi p_{ \pm} D\left(\rho_{ \pm}\right)}{f^{\prime}\left(\rho_{ \pm}\right)}$are positive. Fixing values of $\theta_{0}$ and $\phi_{0}$, 

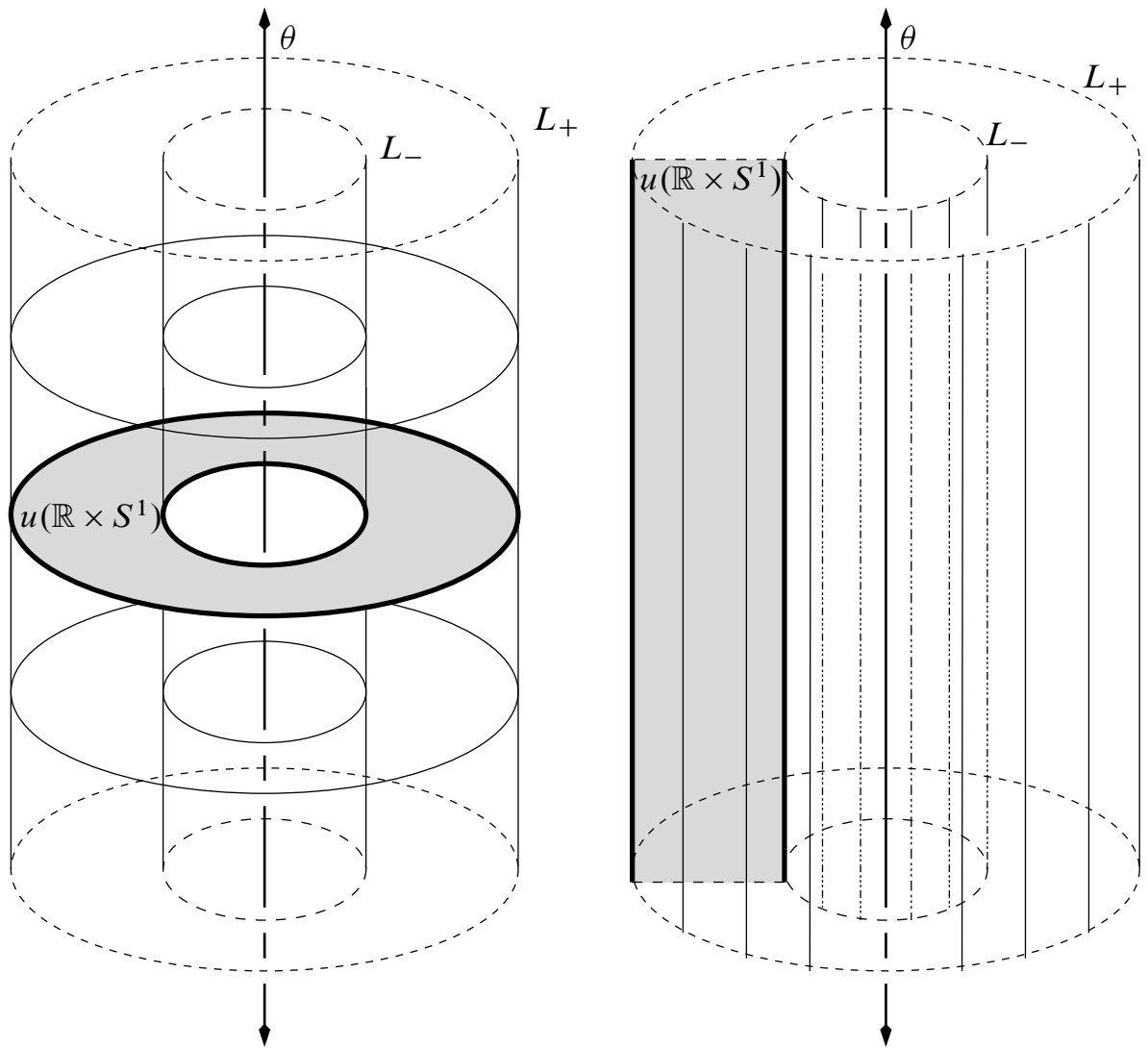

Figure 11: Concentric tori with homologous periodic orbits connected by a finite energy cylinder. On the left is the case where $g^{\prime}\left(\rho_{ \pm}\right)=0$, so the orbits are parallel to $\partial_{\phi}$. On the right, $f^{\prime}\left(\rho_{ \pm}\right)=0$ gives orbits parallel to $\partial_{\theta}$.

suppose $\tilde{u}=(a, u): \mathbb{R} \times S^{1} \rightarrow \mathbb{R} \times M$ is a map of the form

$$
(a(s, t), \theta(s, t), \rho(s, t), \phi(s, t))=\left(a(s), \theta_{0}+q t, \rho(s), \phi_{0}-2 \pi p t\right) .
$$

Then using (35), the Cauchy-Riemann equation for $\tilde{u}$ reduces to the pair of ordinary differential equations

$$
\begin{aligned}
& \frac{d \rho}{d s}=\frac{1}{\beta(\rho)}\left(q f^{\prime}(\rho)-2 \pi p g^{\prime}(\rho)\right), \\
& \frac{d a}{d s}=q f(\rho)-2 \pi p g(\rho) .
\end{aligned}
$$


These have unique solutions for any choice of $\rho(0) \in\left(\rho_{-}, \rho_{+}\right)$and $a(0) \in \mathbb{R}$. Notice that due to our sign convention for $p$ and $q$, the right hand side of (37a) is always positive, thus $\lim _{s \rightarrow \pm \infty} \rho(s)=\rho_{ \pm}$, and we see that $u(s, \cdot)$ converges in $C^{\infty}$ to parameterizations of the orbits $x_{ \pm}$as $s \rightarrow \pm \infty$. It follows then from (1) and Stokes' theorem that $\tilde{u}$ has finite energy $E(\widetilde{u}) \leq T_{+}+T_{-}$. We shall refer to this solution as a cylinder of type $(p, q)$. An example is shown in Figure $13 .^{2}$

It is clear from (37b) that $a$ is a proper function with asymptotically linear growth to $\pm \infty$, as the condition $D(\rho)>0$ guarantees that $\lim _{s \rightarrow \pm \infty} a^{\prime}(s)=q f\left(\rho_{ \pm}\right)-2 \pi p g\left(\rho_{ \pm}\right)$ cannot be zero. This expression determines the sign of the puncture at $s= \pm \infty$ as $\pm \operatorname{sgn}\left(q f\left(\rho_{ \pm}\right)-2 \pi p g\left(\rho_{ \pm}\right)\right)$. To put this in a more revealing form, write $f_{ \pm}:=f\left(\rho_{ \pm}\right)$, $f_{ \pm}^{\prime}:=f^{\prime}\left(\rho_{ \pm}\right)$etc, and observe that by assumption there is a nonzero number

$$
c_{ \pm}=\frac{2 \pi p}{f_{ \pm}^{\prime}}=\frac{q}{g_{ \pm}^{\prime}} .
$$

Then the expression above for the sign becomes

$$
\pm \operatorname{sgn}\left[c_{ \pm}\left(f_{ \pm} g_{ \pm}^{\prime}-f_{ \pm}^{\prime} g_{ \pm}\right)\right]= \pm \operatorname{sgn}\left(c_{ \pm}\right)
$$

since $D\left(\rho_{ \pm}\right)$is positive. Now if both tori $L_{ \pm}$satisfy the Morse-Bott condition, then $0 \neq f_{ \pm}^{\prime} g_{ \pm}^{\prime \prime}-f_{ \pm}^{\prime \prime} g_{ \pm}^{\prime}=-\frac{1}{c_{ \pm}}\left(q f_{ \pm}^{\prime \prime}-2 \pi p g_{ \pm}^{\prime \prime}\right)$, and our sign convention for $p$ and $q$ implies $\operatorname{sgn}\left(q f_{ \pm}^{\prime \prime}-2 \pi p g_{ \pm}^{\prime \prime}\right)=\mp 1$, thus $\operatorname{sgn}\left(f_{ \pm}^{\prime} g_{ \pm}^{\prime \prime}-f_{ \pm}^{\prime \prime} g_{ \pm}^{\prime}\right)=-\operatorname{sgn}\left(c_{ \pm}\right) \operatorname{sgn}\left(q f_{ \pm}^{\prime \prime}-\right.$ $\left.2 \pi p g_{ \pm}^{\prime \prime}\right)= \pm \operatorname{sgn}\left(c_{ \pm}\right)$, and we have

$$
\text { sign of puncture at } L_{ \pm}=\operatorname{sgn}\left(f_{ \pm}^{\prime} g_{ \pm}^{\prime \prime}-f_{ \pm}^{\prime \prime} g_{ \pm}^{\prime}\right) .
$$

This means that in the Morse-Bott case, the sign of a puncture approaching $L_{r}$ is positive if and only if the counterclockwise trajectory $\rho \mapsto(f(\rho), g(\rho))$ is accelerating inward at $\rho=r$, and negative if it accelerates outward.

The equations (37) can be thought of as defining a direction field in the subset $\left(\rho_{-}, \rho_{+}\right) \times$ $\mathbb{R}$ of the $\rho a$-plane, which integrates to a one-dimensional foliation. Since (37b) defines $a(s)$ only up to a constant, this foliation is invariant under the natural $\mathbb{R}$-action on the $a$-coordinate. Meanwhile the set of trajectories $t \mapsto\left(\theta_{0}+q t, \phi_{0}-2 \pi p t\right) \in$ $S^{1} \times \mathbb{R} / 2 \pi \mathbb{Z}$ for all choices of $\theta_{0}$ and $\phi_{0}$ defines another one-dimensional foliation. Putting these together as in (36) creates a two-dimensional foliation of the region $\left\{(a, \theta, \rho, \phi) \in \mathbb{R} \times M \mid \rho \in\left(\rho_{-}, \rho_{+}\right)\right\}$by $\widetilde{J}$-holomorphic cylinders with uniformly bounded energy, and it projects to a one-dimensional foliation of $\left\{\rho \in\left(\rho_{-}, \rho_{+}\right)\right\} \subset M$. We may assume without loss of generality that $f$ and $g$ are chosen so that both tori $L_{\rho_{ \pm}}$are Morse-Bott. Then using the frame $\left(v_{1}, v_{2}\right)$ to trivialize $\xi$ over this region,

\footnotetext{
${ }^{2}$ Thanks to Joel Fish for providing Figure 13 and Figure 14.
} 
it follows from Lemma 2.5 and (6) that each leaf $\tilde{u}$ has $\# \Gamma_{0}=0$ and ind $(\tilde{u})=2$, so Theorem 2.6 implies that the foliation is stable.

We can extend this foliation to $\rho=\rho_{ \pm}$by adding the cylinders over periodic orbits at $L_{ \pm}$. Moreover, if there exists a radius $\rho_{0} \in\left(0, \rho_{-}\right)$such that $\rho_{0}$ and $\rho_{-}$satisfy the same conditions as $\rho_{-}$and $\rho_{+}$, then we can repeat this construction for $\rho \in\left(\rho_{0}, \rho_{-}\right)$ and thus extend the foliation to the region $\rho \in\left[\rho_{0}, \rho_{+}\right]$.

It remains to extend the foliation further toward the center in the case where there is no $\rho<\rho_{-}$with $f^{\prime}(\rho) / 2 \pi g^{\prime}(\rho)=p / q$. To that end, let us redefine our notation with $\rho_{-}=0$ and $L_{+}=\left\{\rho=\rho_{+}\right\}$; choose $\rho_{+}>0$ so that

$$
\frac{f^{\prime}\left(\rho_{+}\right)}{2 \pi g^{\prime}\left(\rho_{+}\right)}=\frac{p}{q} \in \mathbb{Q} \cup\{\infty\} \quad \text { and } \quad \frac{f^{\prime}(\rho)}{2 \pi g^{\prime}(\rho)} \neq \frac{p}{q} \text { for } \rho \in\left(0, \rho_{+}\right) .
$$

Choose the signs of $p$ and $q$ so that $q f^{\prime}-2 \pi p g^{\prime}>0$ for $\rho \in\left(0, \rho_{+}\right)$, and consider once more the family of $\widetilde{J}$-holomorphic cylinders defined by

$$
\tilde{u}=(a, u): \mathbb{R} \times S^{1} \rightarrow \mathbb{R} \times M:(s, t) \mapsto\left(a(s), \theta_{0}+q t, \rho(s), \phi_{0}-2 \pi p t\right),
$$

where $\rho(s)$ and $a(s)$ satisfy the ODEs (37) with $\rho(0) \in\left(0, \rho_{+}\right)$. Once again $u(s, \cdot)$ converges in $C^{\infty}$ as $s \rightarrow \infty$ to some parameterization of a simply covered orbit $P_{+} \subset L_{+}$, and (38) gives the sign of this puncture as

$$
\sigma_{+}:=\operatorname{sgn}\left(f_{+}^{\prime} g_{+}^{\prime \prime}-f_{+}^{\prime \prime} g_{+}^{\prime}\right) .
$$

Define $F(\rho)$ to be the right hand side of (37a). The requirement that $J$ be smooth at $\rho=0$ implies that $\beta(\rho)$ is bounded away from zero as $\rho \rightarrow 0$, thus $\lim _{\rho \rightarrow 0} F(\rho)=0$, and we conclude that $\rho(s) \rightarrow 0$ as $s \rightarrow-\infty$.

We must now distinguish between two cases in order to understand fully the behavior as $s \rightarrow-\infty$. If $q \neq 0, u(s, \cdot)$ converges to the $|q|$-fold cover of $P$, and the sign of the puncture at $-\infty$ is

$$
\sigma_{-}:=-\operatorname{sgn}(q) \cdot \operatorname{sgn}[f(0)]=-\operatorname{sgn}(q) \cdot \operatorname{sgn}\left[g^{\prime \prime}(0)\right],
$$

where we're using the fact that $f(0) g^{\prime \prime}(0)>0$. We can put this in a more geometrically revealing form analogous to (38): observe first that if $P^{|q|}$ is nondegenerate, Proposition 4.6 implies $q f_{-}^{\prime \prime} / 2 \pi g_{-}^{\prime \prime} \notin \mathbb{Z}$ and thus $f_{-}^{\prime \prime} / 2 \pi g_{-}^{\prime \prime} \neq f_{+}^{\prime} / 2 \pi g_{+}^{\prime}=p / q$. Meanwhile our sign convention $q f^{\prime}-2 \pi p g^{\prime}>0$ for $\rho \in\left(0, \rho_{+}\right)$implies

$$
q f_{-}^{\prime \prime}-2 \pi p g_{-}^{\prime \prime}>0 .
$$

This together with the above expression for $\sigma_{-}$yields

$$
\frac{1}{2 \pi} \sigma_{-}\left(\frac{f_{-}^{\prime \prime}}{g_{-}^{\prime \prime}}-\frac{f_{+}^{\prime}}{g_{+}^{\prime}}\right)=\sigma_{-}\left(\frac{f_{-}^{\prime \prime}}{2 \pi g_{-}^{\prime \prime}}-\frac{p}{q}\right)<0,
$$


hence

$$
\sigma_{-}=\operatorname{sgn}\left(\frac{f_{+}^{\prime}}{g_{+}^{\prime}}-\frac{f_{-}^{\prime \prime}}{g_{-}^{\prime \prime}}\right)
$$

Thus when $P^{|q|}$ is nondegenerate, $\sigma_{-}$depends on whether the slope of the trajectory $\rho \mapsto(f(\rho), g(\rho))$ at $\rho=0$ is greater than or less than the slope at $\rho=\rho_{+}$.

We now have a foliation of the region $\rho \in\left(0, \rho_{+}\right)$by an $\mathbb{R}$-invariant family of finite energy cylinders, each convergent to $P^{|q|}$ at one end and a simply covered orbit $P_{+} \subset L_{+}$at the other. These together with the orbit cylinder over $P$ form a finite energy foliation in the region $\left\{\rho<\rho_{+}\right\}$. Figure 12, right, shows an example with $(p, q)=(0,1)$. An example with $p$ and $q$ both nonzero is shown in Figure 14 .

Stability for these cylinders is a somewhat more subtle question than before. Assume $P^{|q|}$ is nondegenerate and $L_{+}$is Morse-Bott. Then since $P^{|q|}$ has odd ConleyZehnder index, Lemma 2.5 and Theorem 2.4 imply that each solution $\tilde{u}$ above has $\operatorname{ind}(\widetilde{u}) \geq 2$; in general however, this inequality can be strict. We claim that the functions $f$ and $g$ can always be adjusted near 0 so that $\operatorname{ind}(\tilde{u})=2$; in this case Theorem 2.6 will imply that the corresponding foliation is stable. Let $\Phi_{0}$ be the symplectic trivialization of $\xi$ along $P$ defined by the Cartesian coordinates, and extend this over $\left\{\rho \leq \rho_{+}\right\}$. Then accounting for the orientation of $\xi$ determined by $\lambda$, we have

$$
\text { wind }_{P_{+}}^{\Phi_{0}}\left(v_{1}\right)=-\sigma_{+} \cdot \operatorname{sgn}[f(0)] \cdot p
$$

and hence

$$
\mu_{\mathrm{CZ}}^{\Phi_{0} \mp}\left(P_{+}\right)=\sigma_{+}(1-2 \operatorname{sgn}[f(0)] \cdot p) .
$$

In order to write $\mu_{\mathrm{CZ}}^{\Phi_{0}}\left(P^{|q|}\right)$ in a convenient form, we compute

$$
\left\lfloor-\frac{|q| f_{-}^{\prime \prime}}{2 \pi g_{-}^{\prime \prime}}\right\rfloor=\left\lfloor-\operatorname{sgn}(q)\left(\frac{q f_{-}^{\prime \prime}-2 \pi p g_{-}^{\prime \prime}}{2 \pi g_{-}^{\prime \prime}}+p\right)\right\rfloor=\left\lfloor\sigma_{-} \frac{q f_{-}^{\prime \prime}-2 \pi p g_{-}^{\prime \prime}}{2 \pi\left|g_{-}^{\prime \prime}\right|}\right\rfloor-\operatorname{sgn}(q) \cdot p .
$$

Then using the index formula in Proposition 4.6,

$$
\begin{aligned}
\operatorname{ind}(\widetilde{u})= & \sigma_{+} \cdot \mu_{\mathrm{CZ}}^{\Phi_{0} \mp}\left(P_{+}\right)+\sigma_{-} \cdot \mu_{\mathrm{CZ}}^{\Phi_{0}}\left(P^{|q|}\right) \\
= & 1-2 \operatorname{sgn}[f(0)] \cdot p+\sigma_{-}\left(2\left\lfloor-\frac{|q| f_{-}^{\prime \prime}}{2 \pi g_{-}^{\prime \prime}}\right\rfloor+1\right) \\
= & 1+\sigma_{-}-(2 \operatorname{sgn}[f(0)] \cdot p)-\left(2 \sigma_{-} \cdot \operatorname{sgn}(q) \cdot p\right) \\
& +2 \sigma_{-}\left\lfloor\sigma_{-} \frac{q f_{-}^{\prime \prime}-2 \pi p g_{-}^{\prime \prime}}{2 \pi\left|g_{-}^{\prime \prime}\right|}\right\rfloor \\
= & 1+\sigma_{-}+2 \sigma_{-}\left\lfloor\sigma_{-} \frac{q f_{-}^{\prime \prime}-2 \pi p g_{-}^{\prime \prime}}{2 \pi\left|g_{-}^{\prime \prime}\right|}\right\rfloor .
\end{aligned}
$$


If $\sigma_{-}=1$ this gives

$$
\operatorname{ind}(\widetilde{u})=2+2\left\lfloor\frac{q f_{-}^{\prime \prime}-2 \pi p g_{-}^{\prime \prime}}{2 \pi\left|g_{-}^{\prime \prime}\right|}\right\rfloor
$$

or if $\sigma_{+}=-1$,

$$
\operatorname{ind}(\widetilde{u})=2\left\lceil\frac{q f_{-}^{\prime \prime}-2 \pi p g_{-}^{\prime \prime}}{2 \pi\left|g_{-}^{\prime \prime}\right|}\right\rceil,
$$

where $\lceil x\rceil$ denotes the smallest integer $\geq x$. Recalling $q f_{-}^{\prime \prime}-2 \pi p g_{-}^{\prime \prime}>0$, we see indeed that in both cases $\operatorname{ind}(\widetilde{u}) \geq 2$, with equality if and only if $q f_{-}^{\prime \prime}-2 \pi p g_{-}^{\prime \prime}$ is sufficiently small. This can always be achieved by adjusting the slope of the trajectory $\rho \mapsto(f(\rho), g(\rho))$ for $\rho$ near 0 , without creating any new points at which $q f^{\prime}(\rho)-$ $2 \pi p g^{\prime}(\rho)=0$. The key point is to make the slopes of the trajectory $\rho \mapsto(f(\rho), g(\rho))$ at $\rho=0$ and $\rho=\rho_{+}$as close as possible.

If on the other hand $q=0$, we have $p=-\operatorname{sgn}\left(g_{-}^{\prime \prime}\right)=-\operatorname{sgn}[f(0)]= \pm 1$ and $\lim _{s \rightarrow-\infty} u(s, t)=\left(\theta_{0}, 0\right) \in P \subset S^{1} \times \mathbb{R}^{2}$. In fact, since

$$
\lim _{\rho \rightarrow 0} F^{\prime}(\rho)=-\frac{2 \pi p g^{\prime \prime}(0)}{\lim _{\rho \rightarrow 0} \beta(\rho)} \neq 0,
$$

one can easily show that $\rho(s)$ converges exponentially fast to 0 , and plugging this behavior into the equation $\rho^{\prime}=F(\rho)$, so does its derivative. We now claim that $a(s)$ is bounded at $-\infty$. For this it suffices to prove that the integral

$$
\int_{-\infty}^{0} \frac{d a}{d s} d s=-2 \pi p \int_{-\infty}^{0} g(\rho(s)) d s
$$

converges. We know $\rho^{\prime}(s)$ satisfies a bound of the form $\left|\rho^{\prime}(s)\right| \leq M e^{\lambda s}$ with $\lambda>0$. Since $g^{\prime}$ is continuous and $\rho$ stays within a bounded interval for all $s$, we have

$$
\begin{aligned}
|g(\rho(s))|=\left|\int_{-\infty}^{s} \frac{d}{d \sigma} g(\rho(\sigma)) d \sigma\right| & \leq \int_{-\infty}^{s}\left|g^{\prime}(\rho(\sigma))\right|,\left|\rho^{\prime}(\sigma)\right| d \sigma \\
& \leq M_{1} \int_{-\infty}^{s} e^{\lambda \sigma} d \sigma=M_{2} e^{\lambda s}
\end{aligned}
$$

for some constant $M_{2}>0$. Then $\int_{-\infty}^{0}|g(\rho(s))| d s<\infty$ and the claim follows. It's clear now that $\tilde{u}$ has finite area as $s \rightarrow-\infty$, thus Gromov's removable singularity theorem implies that $\tilde{u}$ can be extended smoothly to a finite energy plane $\widetilde{v}=(b, v): \mathbb{C} \rightarrow \mathbb{R} \times M$ with $\widetilde{v}\left(e^{2 \pi(s+i t)}\right)=\tilde{u}(s, t)$ and $v(0)=\left(\theta_{0}, 0\right) \in S^{1} \times \mathbb{R}^{2}$. The set of all such planes forms a finite energy foliation in the region $\left\{\rho<\rho_{+}\right\}$. Each is positively asymptotic to a simply covered orbit $P_{+} \subset L_{+}$, and transverse to the central orbit $P$ (Figure 12, left). From (40), we find

$$
\operatorname{ind}(\widetilde{v})=\mu_{\mathrm{CZ}}^{\Phi_{0}-}\left(P_{+}\right)-\chi(\mathbb{C})=(1-(-2))-1=2,
$$



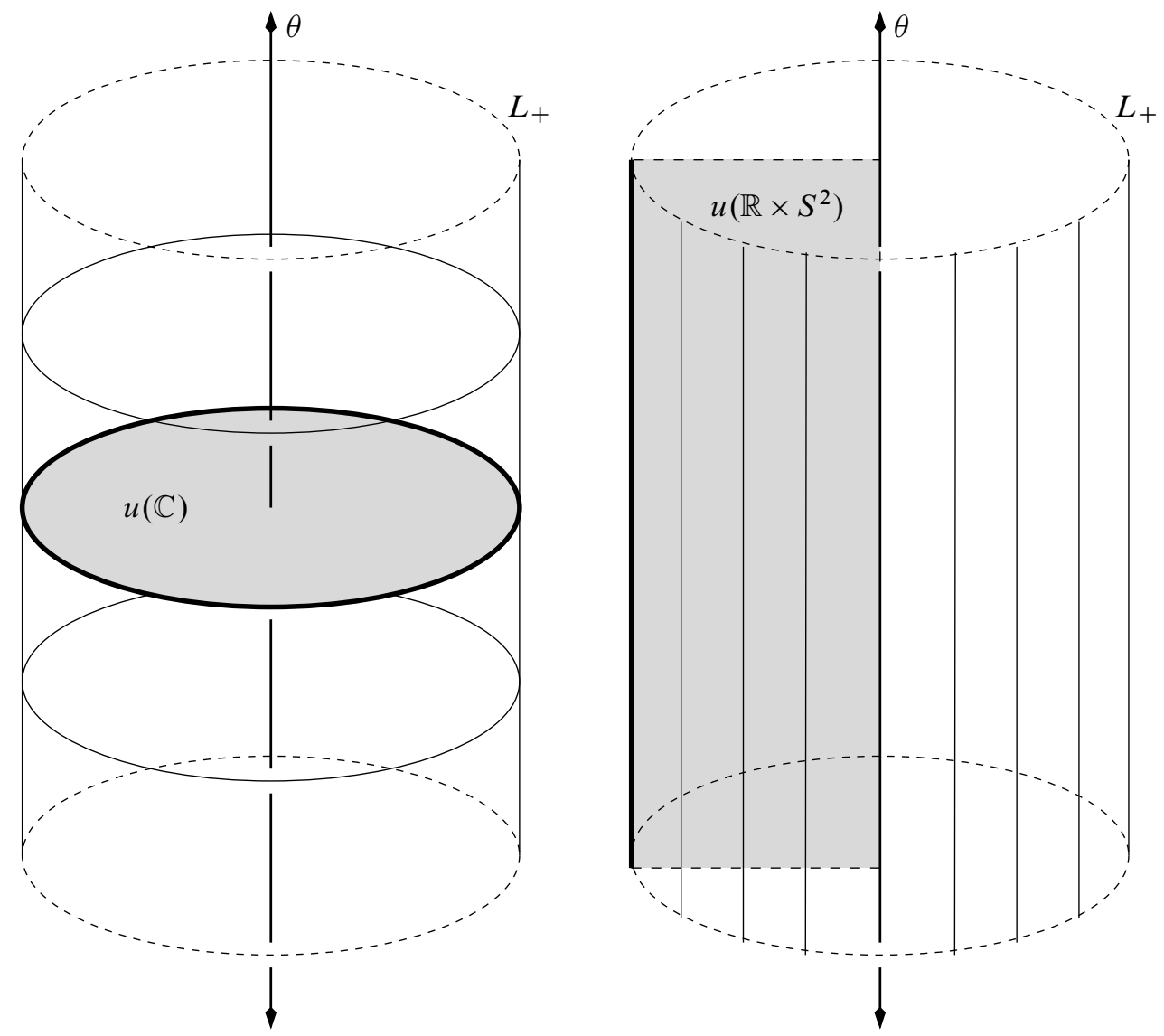

Figure 12: Holomorphic curves inside the innermost torus. If orbits on $L_{+}$ have nontrivial $\partial_{\theta}$ component (right), we get finite energy cylinders with a puncture asymptotic to the central axis; else that puncture is removable (left) and we get a finite energy plane.

so the foliation is stable.

We now apply these constructions to a contact manifold $\left(M, \xi_{K}\right)$ obtained from $\left(S^{3}, \xi\right)$ by a twist surgery along a knot $K$. Let $N \subset S^{3}$ be the corresponding solid torus neighborhood of $K$, identified with $S^{1} \times \overline{B_{\epsilon}^{2}(0)}$, and denote by $N^{\prime}=S^{1} \times \overline{B_{\epsilon}^{2}(0)} \subset M$ the solid torus that replaces it after surgery; thus $M \backslash N^{\prime}=S^{3} \backslash N$. On $N^{\prime}, \xi_{K}$ is the kernel of $\lambda_{K}=f_{K}\left(\rho^{\prime}\right) d \theta^{\prime}+g_{K}\left(\rho^{\prime}\right) d \phi^{\prime}$, which for $\rho \in[\delta, \epsilon]$ is the pull back of $\lambda_{1}=f_{1}(\rho) d \theta+g_{1}(\rho) d \phi$ on $N$ via the gluing map. Let $\rho_{1} \in(\delta, \epsilon)$ be the largest radius for which $g_{1}^{\prime}\left(\rho_{1}\right)=0$, so the Reeb orbits on the torus $L_{\rho_{1}}:=\left\{\rho=\rho_{1}\right\}$ are 


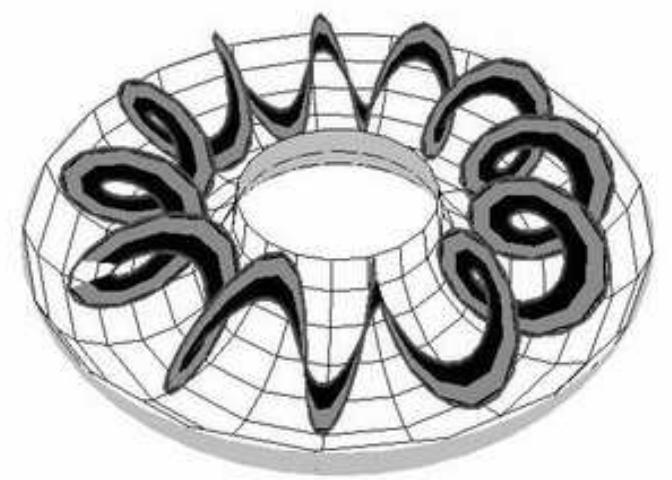

Figure 13: A cylinder of type $(p, q)$ in $S^{1} \times \mathbb{R}^{2}$ with $\rho_{+}>\rho_{-}>0$.

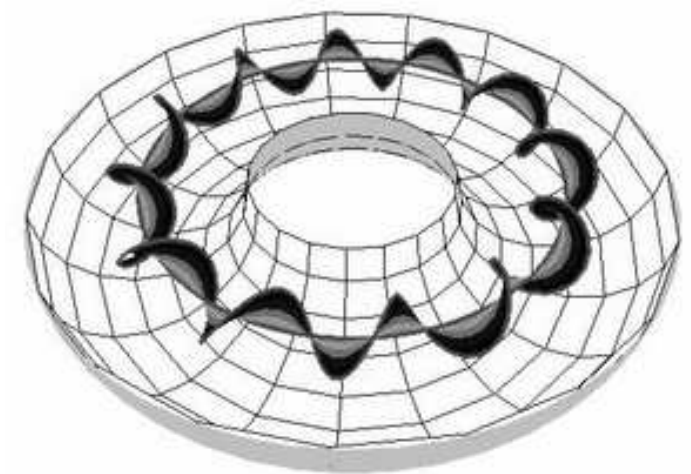

Figure 14: A cylinder of type $(p, q)$ in $S^{1} \times \mathbb{R}^{2}$ with $\rho_{+}>\rho_{-}=0$.

negatively oriented meridians on $N$. They are generally not meridians on $N^{\prime}$, but will represent the homology class $q \lambda^{\prime}-n \mu^{\prime} \in H_{1}\left(L_{\rho_{1}}\right)$, where $\lambda^{\prime}$ and $\mu^{\prime}$ are the standard longitude and meridian respectively for $N^{\prime}$, and $q$ and $n$ are the integers appearing in the matrix (30). There is then a finite set of radii

$$
\rho_{1}>\rho_{2}>\cdots>\rho_{s}>0
$$

for which the Reeb orbits on $L_{\rho_{j}}$ represent classes $\pm\left(q \lambda^{\prime}-n \mu^{\prime}\right) \in H_{1}\left(L_{\rho_{j}}\right)$, and we can foliate the regions in between each of these concentric tori by cylinders of type $(n, q)$. For the region $\left\{\rho \in\left(0, \rho_{s}\right)\right\}$, we obtain planes if $q=0$, otherwise cylinders asymptotic to the $|q|$-fold cover of the orbit $S^{1} \times\{0\} \subset N^{\prime}$. 
By Proposition 4.4, we're free to assume without loss of generality that $q$ is either 0 or \pm 1 , in which case all the orbits in this foliation are simply covered. By conditions (3) and (4) in the proposition, we can also assume the tori $L_{\rho_{j}}$ are all Morse-Bott, the orbit $S^{1} \times\{0\}$ is nondegenerate, all punctures approaching these orbits are positive and the cylinders in the innermost region have index 2 .

Before turning to more global considerations, we note another useful local result, which allows a kind of "analytic continuation" for some foliations.

Proposition 4.7 Let $M=S^{1} \times B_{\epsilon}^{2}(0)$ with contact form $\lambda=f(\rho) d \theta+g(\rho) d \phi$ and $J v_{1}=\beta(\rho) v_{2}$ defining the almost complex structure $\tilde{J}$ on $\mathbb{R} \times M$. Now for some $\delta \in(0, \epsilon)$, choose new smooth functions $f_{1}, g_{1}$ and $\beta_{1}$ which match $f, g$ and $\beta$ for $\rho \in[\delta, \epsilon)$, such that $f_{1} g_{1}^{\prime}-f_{1}^{\prime} g_{1}>0$ for $\rho \in(0, \epsilon)$ and $\beta_{1}(0)>0$. These define new data $\lambda_{1}, J_{1}$ and $\widetilde{J}_{1}$, which are smooth on $M \backslash\left(S^{1} \times\{0\}\right)$ but not necessarily at $S^{1} \times\{0\}$. Suppose there is a neighborhood $\mathcal{U}$ of $S^{1} \times \overline{B_{\delta}^{2}(0)}$ in $M$ and a family of $\tilde{J}$-holomorphic finite energy half-cylinders $\tilde{u}=(a, u):(-\infty, 0] \times S^{1} \rightarrow \mathbb{R} \times M$, all asymptotic to a particular cover of the orbit $P:=S^{1} \times\{0\}$, and defining a finite energy foliation on $\mathcal{U}$. Assume moreover that either of the following is true:

(1) $f^{\prime} g^{\prime \prime}-f^{\prime \prime} g^{\prime} \equiv f_{1}^{\prime} g_{1}^{\prime \prime}-f_{1}^{\prime \prime} g_{1}^{\prime} \equiv 0$ or

(2) $u(s, t)=(\theta(s, t), \rho(s, t), \phi(s, t))$ satisfies $\theta_{s} \phi_{t}-\theta_{t} \phi_{s} \equiv 0$.

Then for each $\tilde{u}$ there is a unique $\widetilde{J}_{1}$-holomorphic half-cylinder $\tilde{u}_{1}(s, t)$ which matches $\tilde{u}$ on some annulus of the form $\left[-s_{0}, 0\right] \times S^{1}$. If additionally $\left(f_{1}, g_{1}\right)$ are $C^{1}$-close to $(f, g)$ then the new maps $\tilde{u}_{1}$ are proper and asymptotic to the same orbit as $\tilde{u}$, and they form an $\mathbb{R}$-invariant foliation of $\mathbb{R} \times(\mathcal{U} \backslash P)$, projecting to a foliation of $\mathcal{U} \backslash P$.

Proof Writing the given curves as $\tilde{u}(s, t)=(a(s, t), \theta(s, t), \rho(s, t), \phi(s, t))$, there are constants $\theta_{0}, \phi_{0}$ and fixed integers $p$ and $q$ such that

$$
\theta(s, t) \rightarrow \theta_{0}+q t, \quad \phi(s, t) \rightarrow \phi_{0}-2 \pi p t \quad \text { as } s \rightarrow-\infty,
$$

and the functions $(a, \theta, \rho, \phi)$ satisfy (35). Combining this with the expressions $a_{s t}-$ $a_{t s}=0$ and $\rho_{s t}-\rho_{t s}=0$ implies

$$
\begin{aligned}
f \Delta \theta+g \Delta \phi & =0, \\
f^{\prime} \Delta \theta+g^{\prime} \Delta \phi-\frac{1}{\beta}\left(f^{\prime} g^{\prime \prime}-f^{\prime \prime} g^{\prime}\right)\left(\theta_{s} \phi_{t}-\theta_{t} \phi_{s}\right) & =0,
\end{aligned}
$$

where $\Delta:=\partial_{s s}+\partial_{t t}$, and $f, g$ and $\beta$ are understood to depend on $\rho(s, t)$. We now seek a map of the form

$$
\tilde{u}_{1}(s, t)=\left(a_{1}(s, t), \theta(s, t), \rho_{1}(s, t), \phi(s, t)\right)
$$


such that $a_{1}(s, t)=a(s, t)$ and $\rho_{1}(s, t)=\rho(s, t)$ for $s \geq-s_{0}$, and solving the corresponding Cauchy-Riemann equations with respect to $f_{1}, g_{1}$ and $\beta_{1}$. Since $\theta(s, t)$ and $\phi(s, t)$ are now fixed functions, the new equations for $\rho_{1}$ in (35) can be interpreted as saying that the graph $\Gamma_{\rho_{1}}:=\left\{\left(s, t, \rho_{1}(s, t)\right)\right\}$ is tangent to a certain 2-plane distribution in $(-\infty, 0] \times S^{1} \times \mathbb{R}$. This distribution turns out to be integrable if and only if

$$
f_{1}{ }^{\prime} \Delta \theta+g_{1}{ }^{\prime} \Delta \phi-\frac{1}{\beta_{1}}\left({f_{1}}^{\prime} g_{1}{ }^{\prime \prime}-f_{1}{ }^{\prime \prime} g_{1}{ }^{\prime}\right)\left(\theta_{s} \phi_{t}-\theta_{t} \phi_{s}\right) \equiv 0,
$$

where the expression is to be understood as a function of three independent variables $(s, t, \rho) \in(-\infty, 0] \times S^{1} \times \mathbb{R}$. If it vanishes identically then solutions $\rho_{1}(s, t)$ exist locally. Assume this for the moment: then choosing $s_{0} \in(-\infty, 0)$ such that $\rho(s, t) \geq \delta$ for all $s \geq s_{0}$, there is a solution $\rho_{1}(s, t)$ on $\left(-s_{1}, 0\right] \times S^{1}$ for some $-s_{1}<-s_{0}$, with $\rho_{1}(s, t)=\rho(s, t)$ for $s \geq-s_{0}$. For topological reasons, the continued solution is automatically 1 -periodic in $t$. Then for fixed $t$, the function $s \mapsto \rho_{1}(s, t)$ satisfies the ODE

$$
\frac{d \rho_{1}}{d s}=\frac{1}{\beta_{1}\left(\rho_{1}\right)}\left(f_{1}^{\prime}\left(\rho_{1}\right) \theta_{t}+g_{1}^{\prime}\left(\rho_{1}\right) \phi_{t}\right),
$$

and we see that the solution $\rho_{1}(s, t)$ extends to $(-\infty, 0] \times S^{1}$ with

$$
\lim _{s \rightarrow-\infty} \rho_{1}(s, t)=\rho_{0},
$$

where $\rho_{0} \geq 0$ is the largest radius at which $f_{1}^{\prime}\left(\rho_{0}\right) / 2 \pi g_{1}^{\prime}\left(\rho_{0}\right)=p / q$, or zero if there is no such radius. The latter is necessarily the case, in particular, if $\left(f_{1}, g_{1}\right)$ is $C^{1}$-close to $(f, g)$, because the same argument for $\tilde{u}$ shows that $(f, g)$ cannot admit any radius at which this relation is satisfied.

The remaining two equations in (35) specify the gradient of $a_{1}(s, t)$ in terms of known functions, so solutions exist locally if and only if this gradient is curl-free, which in this case means

$$
f_{1} \Delta \theta+g_{1} \Delta \phi \equiv 0
$$

for all $(s, t) \in(-\infty, 0] \times S^{1}$ and $\rho=\rho_{1}(s, t)$. There is then a unique solution on $(-\infty, 0] \times S^{1}$ with $a_{1}(s, t)=a(s, t)$ for all $s \geq s_{0}$, and another ODE argument establishes that in the case $\rho_{0}=0, a(s, t)$ blows up linearly as $s \rightarrow-\infty$.

We claim that the integrability conditions are satisfied whenever either of the two additional assumptions in the Proposition are met. Indeed, if $f^{\prime} g^{\prime \prime}-f^{\prime \prime} g^{\prime} \equiv f_{1}{ }^{\prime} g_{1}{ }^{\prime \prime}-$ $f_{1}{ }^{\prime \prime} g_{1}{ }^{\prime} \equiv 0$, then (41) and (42) give $f \Delta \theta+g \Delta \phi=f^{\prime} \Delta \theta+g^{\prime} \Delta \phi=0$, and since the contact condition requires $(f, g)$ and $\left(f^{\prime}, g^{\prime}\right)$ to be linearly independent in $\mathbb{R}^{2}$ for all $\rho$, we conclude that both $\theta(s, t)$ and $\phi(s, t)$ are harmonic. Thus (43) and (44) are 
satisfied for all $(s, t, \rho)$. In the other case, $\theta_{s} \phi_{t}-\theta_{t} \phi_{s} \equiv 0$ together with (41) and (42) implies again that $\theta$ and $\phi$ are harmonic, so the same argument applies.

\subsection{Surgery on a holomorphic open book}

4.3.1 $\mathcal{F}_{0} \rightarrow \mathcal{F}_{1}$ : Stabilizing an open book decomposition The global construction begins with a stable foliation of open book type on the tight 3-sphere. Such foliations follow from a general existence result in Hofer-Wysocki-Zehnder [15], but for our purposes, we can produce one using much less machinery.

Define $\lambda_{0}$ on $S^{3}$ as in (31): then the Reeb vector field $X_{0}$ generates the Hopf fibration. At each $z \in S^{3}, \xi_{0}=\operatorname{ker} \lambda_{0}$ is the unique complex line in $T_{z} S^{3} \subset \mathbb{C}^{2}$, which therefore admits a natural complex multiplication $i \in \Gamma(\operatorname{End}(\xi))$. Let $\widetilde{J}_{0}$ be the $\mathbb{R}$-invariant almost complex structure on $\mathbb{R} \times S^{3}$ associated to $\lambda_{0}$ and $i$. Then the diffeomorphism

$$
\Phi:\left(\mathbb{R} \times S^{3}, \widetilde{J}_{0}\right) \rightarrow\left(\mathbb{C}^{2} \backslash\{0\}, i\right):(a, m) \mapsto e^{2 a} m
$$

is biholomorphic. For each $\zeta \in \mathbb{C} \backslash\{0\}$, we now define a $\widetilde{J}_{0}$-holomorphic plane

$$
\tilde{u}_{\zeta}=\left(a_{\zeta}, u_{\zeta}\right): \mathbb{C} \rightarrow \mathbb{R} \times S^{3}: z \mapsto \Phi^{-1}(z, \zeta),
$$

and for $\zeta=0$, a cylinder (ie punctured plane)

$$
\tilde{u}_{0}=\left(a_{0}, u_{0}\right): \mathbb{C} \backslash\{0\} \rightarrow \mathbb{R} \times S^{3}: z \mapsto \Phi^{-1}(z, \zeta) .
$$

The latter is in fact the trivial cylinder over the Hopf circle

$$
P_{\infty}:=\left\{\left(e^{2 \pi i \theta}, 0\right) \mid \theta \in S^{1}\right\},
$$

and the collection of planes $\left\{\tilde{u}_{\zeta}\right\} \zeta \in \mathbb{C} \backslash\{0\}$ is an $\mathbb{R}$-invariant 2-parameter family of embedded, pairwise disjoint finite energy planes asymptotic to $P_{\infty}$. Altogether these define a finite energy foliation $\mathcal{F}_{0}$ on $\left(S^{3}, \lambda_{0}, i\right)$. The projection to $S^{3}$ is a planar open book decomposition with one binding orbit.

The foliation $\mathcal{F}_{0}$ is not stable, because the degeneracy of $P_{\infty}$ gives the planes index 4. We can fix this with a small change to $\lambda_{0}$ near $P_{\infty}$, using Proposition 4.7. Indeed, pick any $R \leq 1 / \sqrt{2 \pi}$ and identify a neighborhood of $P_{\infty}$ with $S^{1} \times B_{R}^{2}(0)$ via the embedding

$$
\psi: S^{1} \times B_{R}^{2}(0) \hookrightarrow S^{3}:(\theta, \rho, \phi) \mapsto e^{2 \pi i \theta}\left(\sqrt{1-2 \pi \rho^{2}}, e^{i \phi} \sqrt{2 \pi} \rho\right) .
$$

Then $\psi\left(S^{1} \times\{0\}\right)=P_{\infty}$ and $\psi^{*} \lambda_{0}=\pi\left(d \theta+\rho^{2} d \phi\right)=f(\rho) d \theta+g(\rho) d \phi$, where $f(\rho)=\pi$ and $g(\rho)=\pi \rho^{2}$. Defining the vector fields $v_{1}$ and $v_{2}$ as in (32), the 
complex multiplication is now specified by $i v_{1}=\beta(\rho) v_{2}$, where

$$
\beta(\rho)=\frac{2 \pi}{1-2 \pi \rho^{2}} .
$$

For $\zeta=r e^{i \phi_{0}} \in \mathbb{C} \backslash\{0\}$, we can express the asymptotic behavior of the holomorphic plane $\tilde{u}_{\zeta}(z)=\left(a_{\zeta}(z), u_{\zeta}(z)\right)=\left(\frac{1}{2} \ln |(z, \zeta)|, \frac{(z, \zeta)}{|(z, \zeta)|}\right)$ in these coordinates by

$$
\begin{aligned}
& (a(s, t), \theta(s, t), \rho(s, t), \phi(s, t)):=\left(a\left(e^{-2 \pi(s+i t)}\right), \psi^{-1} \circ u_{\zeta}\left(e^{-2 \pi(s+i t)}\right)\right) \\
& \quad=\left(\frac{1}{4} \ln \left(e^{-4 \pi s}+r^{2}\right),-t, \frac{r}{\sqrt{2 \pi\left(e^{-4 \pi s}+r^{2}\right)}}, \phi_{0}+2 \pi t\right),
\end{aligned}
$$

with $(s, t) \in\left(-\infty, s_{0}\right] \times S^{1}$ for $s_{0}$ sufficiently close to $-\infty$. Observe now that $\theta_{s} \phi_{t}-\theta_{t} \phi_{s} \equiv 0$, thus by Proposition 4.7, any $C^{1}$-small change in $f$ and $g$ for $\rho$ near 0 admits a new foliation, which is identical to $\mathcal{F}$ outside some neighborhood of $P_{\infty}$. In particular, pick $\delta \in(0, R)$ and define

$$
\lambda_{1}=f_{1}(\rho) d \theta+g_{1}(\rho) d \phi=h(\rho) \cdot\left(\pi d \theta+\pi \rho^{2} d \phi\right)
$$

for some function $h$ that satisfies $h(\rho)=1$ for $\rho \geq \delta$ and is $C^{1}$-close to this on $[0, R)$, and such that $h^{\prime \prime}(0)$ is small but positive. Then a calculation using Proposition 4.6 shows that for the new contact form, $P_{\infty}$ is a nondegenerate orbit with $\mu_{\mathrm{CZ}}\left(P_{\infty}\right)=3$. The new family of planes asymptotic to $P_{\infty}$ then have index 2 and form a stable foliation $\mathcal{F}_{1}$ on $\left(S^{3}, \lambda_{1}, i\right)$.

4.3.2 $\mathcal{F}_{1} \rightarrow \mathcal{F}_{2}$ : Fixing $\lambda$ and $J$ near a link Next, introduce a positively transverse link $K=K_{1} \cup \cdots \cup K_{n} \subset S^{3}$. By Lemma 4.5, there are smooth families $\gamma_{j}^{\tau}: S^{1} \rightarrow M$ for $\tau \in[0,1]$ such that $\gamma_{j}^{1}\left(S^{1}\right)=K_{j}, \gamma_{j}^{0}(t)=\left(0, e^{2 \pi i k_{j} t}\right)$ for some $k_{j} \in \mathbb{N}$, and for each fixed $\tau \in(0,1]$, the maps $\gamma_{1}^{\tau}, \ldots, \gamma_{n}^{\tau}: S^{1} \rightarrow M$ are mutually non-intersecting embeddings transverse to $\xi$. Denote $K_{j}^{\tau}=\gamma_{j}^{\tau}\left(S^{1}\right)$ and $K^{\tau}=K_{1}^{\tau} \cup \cdots \cup K_{N}^{\tau}$ for $\tau \in(0,1]$.

Lemma 4.8 For $\tau>0$ sufficiently small, there is a contact form $\lambda_{2}$ with $\operatorname{ker} \lambda_{2}=\xi_{0}$ and the following properties:

(i) $\lambda_{2}$ is $C^{1}$-close to $\lambda$, and differs from $\lambda$ only in an arbitrarily small neighborhood of $K^{\tau}$,

(ii) Each of the knots $K_{j}^{\tau}$ has a tubular neighborhood $N_{j} \cong S^{1} \times B_{\epsilon}^{2}(0)$ with coordinates $(\theta, \rho, \phi)$ in which $K_{j}^{\tau}=\{\rho=0\}$ and $\lambda_{2}=c\left(d \theta+\rho^{2} d \phi\right)$ for some constant $c>0$. 
For a complete proof, we refer to [29, Proposition 5.1.3]. The main idea is as follows: observe first that a neighborhood of $P_{0}:=\left\{\left(0, e^{2 \pi i \theta}\right) \mid \theta \in S^{1}\right\}$ admits coordinates in which $\lambda_{1}=c\left(d \theta+\rho^{2} d \phi\right)$. These are defined by an embedding $\Psi_{0}: S^{1} \times B_{\epsilon}^{2}(0) \hookrightarrow S^{3}$ quite similar to (45). One can then use a parameterized version of the Moser deformation argument to construct for each $K_{j}$ a family of contact immersions $\psi_{j}^{\tau}: S^{1} \times B_{\epsilon}^{2}(0) \rightarrow$ $S^{3}$, which are embeddings near $S^{1} \times\{0\}$ for $\tau>0$, taking $S^{1} \times\{0\}$ to $K_{j}^{\tau}$, and converge as $\tau \rightarrow 0$ to a $k_{j}$-fold cover of $\Psi_{0}$. These define coordinate neighborhoods near $K_{j}^{\tau}$ in which $\lambda_{1}$ is $C^{1}$-close to something of the form $c\left(d \theta+\rho^{2} d \phi\right)$.

Let us now redefine notation and call $K^{\tau}$ (for sufficiently small $\tau>0$ ) simply $K$; we can then assume there is a contact form $\lambda_{2}$ as in Lemma 4.8, taking the form $c\left(d \theta+\rho^{2} d \phi\right)$ in coordinates near each component of $K$. Choose a smooth homotopy of contact forms $\left\{\lambda_{r}\right\}_{r \in[1,2]}$ such that $\operatorname{ker} \lambda_{r}=\xi_{0}$ for all $r$, and each $\lambda_{r}$ is $C^{1-}$ close to $\lambda_{1}$, differing from $\lambda_{1}$ only in a tubular neighborhood of $K$. Observe that the corresponding Reeb vector fields $X_{r}$ are are all $C^{0}$-close to $X_{1}$, and equal to it outside a compact neighborhood of $K$. We may therefore assume $X_{r}$ is always transverse to the projection of the foliation $\mathcal{F}_{1}$ on $S^{3} \backslash P_{\infty}$. As a consequence we have, without loss of generality the following Proposition.

Proposition 4.9 Every periodic orbit of $X_{r}$ that's geometrically distinct from $P_{\infty}$ is nontrivially linked with $P_{\infty}$.

For $r \in[1,2]$, choose also a smooth homotopy of admissible complex multiplications $J_{r}: \xi_{0} \rightarrow \xi_{0}$ such that $J_{1} \equiv i, J_{r}$ differs from $i$ only in a neighborhood of $K$, and $J_{2}$ is defined in the coordinates $(\theta, \rho, \phi)$ near each component of $K$ by a relation of the form $J_{2} v_{1}=\beta(\rho) v_{2}$, as in Section 4.2. These choices define a smooth homotopy of almost complex structures $\widetilde{J}_{r}$. Observe that the binding orbit $P_{\infty}$ remains a closed orbit with $\mu_{\mathrm{CZ}}\left(P_{\infty}\right)=3$ for all $r$. We can now use the machinery of Section 2 and Section 3 to show that the foliation $\mathcal{F}_{1}$ extends to a continuous family of foliations for $r \in[1,2]$.

Proposition 4.10 For each $r \in[1,2]$, there exists a stable finite energy foliation $\mathcal{F}_{r}$ of $\left(S^{3}, \lambda_{r}, J_{r}\right)$ which projects to an open book decomposition of $S^{3}$, with binding orbit $P_{\infty}$

Proof Denote by $\mathcal{M}_{r}$ the moduli space of all $\widetilde{J}_{r}$-holomorphic finite energy surfaces, and define the space

$$
\mathcal{M}=\left\{(r, \tilde{u}) \mid r \in[1,2], \tilde{u} \in \mathcal{M}_{r}\right\} .
$$

The latter has a natural topology induced by the same notion of convergence as in $\mathcal{M}_{r}$, and there are natural continuous inclusions $\mathcal{M}_{r} \hookrightarrow \mathcal{M}$ for each $r$, as well as a natural 
$\mathbb{R}$-action on $\mathcal{M}$. Let $\mathcal{M}_{1}^{*}$ denote the connected component of $\mathcal{M}_{1}$ that contains the planes in the foliation $\mathcal{F}_{1}$, let $\mathcal{M}^{*}$ be the corresponding connected component of $\mathcal{M}$ containing $\mathcal{M}_{1}^{*}$, and then define $\mathcal{M}_{r}^{*}=\mathcal{M}^{*} \cap \mathcal{M}_{r}$.

Combining Theorem 2.6, Theorem 2.7 and Theorem 2.8, we see that for every $(r, \tilde{u}) \in$ $\mathcal{M}^{*}, \tilde{u}=(a, u)$ is an embedded index 2 plane asymptotic to $P_{\infty}$, and $u: \mathbb{C} \rightarrow S^{3}$ is also embedded. Moreover $\tilde{u}$ is regular, and its neighborhood in $\mathcal{M}_{r}^{*}$ foliates neighborhoods of the images of $\tilde{u}$ and $u$. Clearly then, $\mathcal{M}^{*} / \mathbb{R}$ is a smooth 2-dimensional manifold, for which the projection map

$$
\mathcal{M}^{*} / \mathbb{R} \rightarrow \mathbb{R}:(r,[\tilde{u}]) \mapsto r
$$

is always regular. In light of the linking condition in Proposition 4.9, Theorem 3.4 implies that $\mathcal{M}^{*} / \mathbb{R}$ is compact. It follows that there is a diffeomorphism

$$
\psi:[1,2] \times \mathcal{M}_{1}^{*} / \mathbb{R} \rightarrow \mathcal{M}^{*} / \mathbb{R}
$$

such that $\psi(1,[\tilde{u}])=[\tilde{u}]$ and for each $r \in[1,2], \psi(r, \cdot)$ is a diffeomorphism $\mathcal{M}_{1}^{*} / \mathbb{R} \rightarrow$ $\mathcal{M}_{r}^{*} / \mathbb{R}$. Thus $\mathcal{M}_{r}^{*} / \mathbb{R} \cong \mathcal{M}_{1}^{*} / \mathbb{R} \cong S^{1}$. Applying Theorem 2.6 and Theorem 2.8 again, we see that any two elements of $\mathcal{M}_{r}^{*} / \mathbb{R}$ are either identical or have disjoint images in $S^{3}$. Moreover, if $\mathcal{U}_{r} \subset S^{3} \backslash P_{\infty}$ is the set of points contained in the image of any curve $[\tilde{u}] \in \mathcal{M}_{r}^{*} / \mathbb{R}$, then Theorem 2.6 and Theorem 3.4 together imply that $\mathcal{U}_{r}$ is open and closed, so $\mathcal{U}_{r}=S^{3} \backslash P_{\infty}$. The collection of curves $\mathcal{M}_{r}^{*}$, together with the trivial cylinder over $P_{\infty}$, therefore form a stable finite energy foliation of $\left(S^{3}, \lambda_{r}, J_{r}\right)$.

4.3.3 $\mathcal{F}_{2} \rightarrow \mathcal{F}_{3}$ : Cutting out disks For the remainder of Section 4.3, we impose the following restrictive assumption.

Assumption 4.11 For each component $K_{j} \subset K, \operatorname{lk}\left(K_{j}, P_{\infty}\right)=1$.

This is needed for technical reasons in the arguments that follow, but will be removed in Section 4.4 by a branched covering argument.

By the above results, we have a stable foliation $\mathcal{F}_{2}$ of $\left(S^{3}, \lambda_{2}, J_{2}\right)$, transverse to a link $K=K_{1} \cup \cdots \cup K_{m}$ whose components have disjoint tubular neighborhoods $N_{j} \cong S^{1} \times B_{\epsilon}^{2}(0)$ on which $\lambda_{2}=c_{j}\left(d \theta+\rho^{2} d \phi\right)$ and $J_{2}$ has the form $J_{2} v_{1}=\beta_{j}(\rho) v_{2}$. The Reeb vector field on $N_{j}$ is $X_{2}=\frac{1}{c_{j}} \partial_{\theta}$. Pick $\delta \in(0, \epsilon)$ and let $N_{j}^{\delta}=\{\rho \leq \delta\} \subset N_{j}$, with $L_{j}:=\partial N_{j}^{\delta}$. Observe that since $L_{j}$ is foliated by Reeb orbits, which are necessarily transverse to the leaves of $\mathcal{F}_{2}, L_{j}$ is also transverse to these leaves.

We will now replace the planes in $\mathcal{F}_{2}$ with solutions to a boundary value problem, having boundary mapped to the tori $L_{j}$. For this it is necessary to throw out all except 
one of the curves in $\mathcal{F}_{2}$; we will be able to reconstruct a foliation afterwards. Therefore, pick any finite energy plane $\tilde{u}=(a, u): \mathbb{C} \rightarrow \mathbb{R} \times S^{3}$ which parametrizes a leaf of $\mathcal{F}_{2}$, and define the set of $m$ disjoint open disks

$$
\mathcal{D}_{1} \cup \cdots \cup \mathcal{D}_{m} \subset \mathbb{C}
$$

by $\mathcal{D}_{j}=u^{-1}\left(\right.$ int $\left.N_{j}^{\delta}\right)$. Then

$$
(\Sigma, j):=\left(S^{2} \backslash\left(\mathcal{D}_{1} \cup \cdots \cup \mathcal{D}_{m}\right), i\right)
$$

is a compact Riemann surface with boundary

$$
\partial \Sigma=\gamma_{1} \cup \cdots \cup \gamma_{m}
$$

where $\gamma_{j}:=-\partial \overline{\mathcal{D}}_{j}$, and we have $u\left(\gamma_{j}\right) \subset L_{j}$. Let $\dot{\Sigma}=\Sigma \backslash\{\infty\}$. Observe that due to Assumption 4.11, each torus $L_{j}$ meets the image of a unique component $\gamma_{j}$ under $u$. Then since each Reeb orbit on $L_{j}$ has a single transverse intersection with $u\left(\gamma_{j}\right)$, there are unique smooth functions $g_{j}: L_{j} \rightarrow \mathbb{R}$ such that $d g_{j}\left(X_{2}\right) \equiv 0$ and $a(z)=g_{j}(u(z))$ for all $z \in \gamma_{j}$. Thus $\tilde{u}_{j}$ satisfies the totally real boundary condition

$$
\widetilde{u}\left(\gamma_{j}\right) \subset \widetilde{L}_{j}:=\left\{\left(g_{j}(x), x\right) \in \mathbb{R} \times S^{3} \mid x \in L_{j}\right\} .
$$

This boundary condition is not Lagrangian, so it does not naturally give rise to any obvious energy bounds. ${ }^{3}$ However, the fact that $d g_{j}\left(X_{2}\right) \equiv 0$ will allow us to identify each $\widetilde{L}_{j}$ with a Lagrangian torus in the symplectization of $S^{3}$ with a non-contact stable Hamiltonian structure. This is why Assumption 4.11 is necessary.

Proposition 4.12 Suppose $M$ is an oriented 3-manifold with positive contact form $\lambda$ and Reeb vector field $X$, whose flow is globally defined, and $J$ is an admissible complex multiplication on $\xi=\operatorname{ker} \lambda$ which is preserved by the Reeb flow. Denote by $\widetilde{J}$ the associated almost complex structure on $\mathbb{R} \times M$, and define an $\mathbb{R}$-equivariant diffeomorphism by

$$
\Psi: \mathbb{R} \times M \rightarrow \mathbb{R} \times M:(a, m) \mapsto(a+F(m), m)
$$

for some smooth function $F: M \rightarrow \mathbb{R}$ that satisfies $d F(X) \equiv 0$. Then if $\xi^{\prime} \subset T M$ is the unique 2-plane distribution in $T M=T(\{0\} \times M) \subset T(\mathbb{R} \times M)$ which is preserved by $\Psi_{*} \widetilde{J}$, and $J^{\prime}:=\left.\Psi_{*} \widetilde{J}\right|_{\xi^{\prime}}: \xi^{\prime} \rightarrow \xi^{\prime}$, the data

$$
\mathcal{H}^{\prime}:=\left(\xi^{\prime}, X, d \lambda, J^{\prime}\right)
$$

\footnotetext{
${ }^{3}$ It is shown in [29] that one can choose a new definition of energy so that suitable bounds are satisfied and the compactness argument goes through. Here we follow an alternative and somewhat simpler approach.
} 
define a stable Hamiltonian structure on $M$, for which the associated almost complex structure is precisely $\Psi_{*} \widetilde{J}$.

Proof Denoting by $\pi: T M \rightarrow \xi$ the projection along $X$, define a $1-$ form

$$
\lambda^{\prime}=\lambda-d F \circ J \circ \pi
$$

and let $\xi^{\prime}=\operatorname{ker} \lambda^{\prime}$ (we're not assuming this is the same $\xi^{\prime}$ defined in the statement above). Clearly $\lambda^{\prime}(X) \equiv 1$, and we claim that also $d \lambda^{\prime}(X, \cdot) \equiv 0$. Since $L_{X} \lambda^{\prime}=$ $d \iota_{X} \lambda^{\prime}+\iota_{X} d \lambda^{\prime}=\iota_{X} d \lambda^{\prime}$, this is equivalent to the statement that $\xi^{\prime}$ is preserved by the flow of $X$. Denote this flow by $\varphi^{t}: M \rightarrow M$ and observe that $\varphi_{*}^{t} X \equiv X$ for all $t$, and by assumption similarly $F \circ \varphi^{t} \equiv F$ and $\varphi_{*}^{t} J \equiv J$. For $m \in M$, any $v \in \xi_{m}^{\prime}$ can be written as $v=[d F(m) J \hat{v}] X(m)+\widehat{v}$ where $\widehat{v}:=\pi v \in \xi_{m}$. Then

$$
\begin{aligned}
\varphi_{*}^{t} v & =[d F(m) J \hat{v}] X\left(\varphi^{t}(m)\right)+\varphi_{*}^{t} \widehat{v} \\
& =\left[d\left(F \circ \varphi^{t}\right)(m) J \hat{v}\right] X\left(\varphi^{t}(m)\right)+\varphi_{*}^{t} \widehat{v} \\
& =\left[d F\left(\varphi^{t}(m)\right) \cdot \varphi_{*}^{t}(J \widehat{v})\right] X\left(\varphi^{t}(m)\right)+\varphi_{*}^{t} \widehat{v} \\
& =\left[d F\left(\varphi^{t}(m)\right) \cdot J\left(\varphi_{*}^{t} \widehat{v}\right)\right] X\left(\varphi^{t}(m)\right)+\varphi_{*}^{t} \widehat{v} \in \xi_{\varphi^{t}(m)}^{\prime},
\end{aligned}
$$

proving the claim.

Now observe $d \lambda(X, \cdot) \equiv 0$, and since $\xi^{\prime}$ is transverse to $X, d \lambda$ is nondegenerate on $\xi^{\prime}$ and provides a suitable taming form for any complex multiplication $J^{\prime}: \xi^{\prime} \rightarrow \xi^{\prime}$ with the correct orientation. We show next that $\xi^{\prime}$ is in fact the unique distribution preserved by $\Psi_{*} \widetilde{J}$. Indeed, for $v=[d F(m) J \hat{v}] X(m)+\widehat{v} \in \xi_{m}^{\prime}$, we have

$$
\begin{aligned}
\left(\Psi_{*} \widetilde{J}\right) v & =T \Psi \circ \widetilde{J} \circ T \Psi^{-1}([d F(m) J \hat{v}] X(m)+\widehat{v}) \\
& =T \Psi \circ \widetilde{J}\left([d F(m) J \hat{v}] X(m)-[d F(m) \hat{v}] \partial_{a}+\widehat{v}\right) \\
& =T \Psi\left(-[d F(m) J \hat{v}] \partial_{a}-[d F(m) \hat{v}] X(m)+J \hat{v}\right) \\
& =-[d F(m) J \hat{v}] \partial_{a}-[d F(m) \hat{v}] X(m)+[d F(m) J \hat{v}] \partial_{a}+J \hat{v} \\
& =-[d F(m) \hat{v}] X(m)+J \hat{v} \\
& =[d F(m) J(J \hat{v})] X(m)+J \hat{v} \in \xi_{m}^{\prime},
\end{aligned}
$$

thus $\Psi_{*} \widetilde{J}$ restricts on $\xi^{\prime}$ to the unique map $J^{\prime}: \xi^{\prime} \rightarrow \xi^{\prime}$ such that $\left.J \circ \pi\right|_{\xi^{\prime}} \equiv \pi \circ J^{\prime}$. Finally, observe that $\Psi_{*} \widetilde{J}$ is clearly $\mathbb{R}$-invariant, and since $T \Psi^{-1}$ and $T \Psi$ each preserve both $\partial_{a}$ and $X$,

$$
\left(\Psi_{*} \widetilde{J}\right) \partial_{a}=T \Psi \circ \widetilde{J} \circ T \Psi^{-1}\left(\partial_{a}\right)=X .
$$

Remark 4.13 There is an obvious smooth homotopy between the two stable Hamiltonian structures $\mathcal{H}_{0}:=(\xi, X, d \lambda, J)$ and $\mathcal{H}_{1}:=\left(\xi^{\prime}, X, d \lambda, J^{\prime}\right):$ just define $\mathcal{H}_{\tau}=$ 
$\left(\xi_{\tau}, X, d \lambda, J_{\tau}\right)$ for $\tau \in[0,1]$ by the same trick, but using the functions $F_{\tau}:=\tau F: M \rightarrow$ $\mathbb{R}$.

Remark 4.14 Finding nontrivial examples of the situation in Proposition 4.12 requires very precise knowledge of the Reeb dynamics. One interesting example is the case where $M$ is a principal $S^{1}$-bundle over a Riemann surface with compatible symplectic structure, and the fibers are generated by $X$ (cf [3, Example 2.2]). Then the choice of $F$ determines $\xi^{\prime}$ as a principal connection on this bundle.

We apply the above idea as follows. Pick a smooth function $F: S^{3} \rightarrow \mathbb{R}$ supported in $N_{1} \cup \cdots \cup N_{m}$, such that $d F\left(X_{2}\right) \equiv 0$ and $F(x)=-g_{j}(x)$ for all $x \in L_{j}$. Then the diffeomorphism (46) satisfies

$$
\Psi\left(\tilde{L}_{j}\right)=\{0\} \times L_{j},
$$

and there is a stable Hamiltonian structure $\mathcal{H}_{3}=\left(\xi_{2}^{\prime}, X_{2}, d \lambda_{2}, J_{2}^{\prime}\right)$ with associated almost complex structure $\widetilde{J}_{3}$ such that

$$
\tilde{v}=(b, v):=\Psi \circ \tilde{u}: \dot{\Sigma} \rightarrow \mathbb{R} \times S^{3}
$$

is $\widetilde{J}_{3}$-holomorphic and satisfies the Lagrangian boundary condition $\widetilde{v}\left(\gamma_{j}\right) \subset\{0\} \times L_{j}$. Thus writing $\Lambda=\left(\{0\} \times L_{1}, \ldots,\{0\} \times L_{m}\right)$, we have $\widetilde{v} \in \mathcal{M}_{\mathcal{H}_{3}, \Lambda}$; in fact $\widetilde{v}$ satisfies the same assumptions as the sequence in our main compactness theorem, so Lemma 3.2 implies $\operatorname{ind}(\widetilde{v})=2$. Then by Theorem 2.6, the connected component $\mathcal{M}_{3}^{*} \subset \mathcal{M}_{\mathcal{H}_{3}, \Lambda}$ containing $\widetilde{v}$ is a smooth 2 -manifold with free and proper $\mathbb{R}$-action, so $\mathcal{M}_{3}^{*} / \mathbb{R}$ is a smooth 1-manifold, and Theorem 3.4 implies it is compact, ie it is diffeomorphic to $S^{1}$. Arguing again as in Proposition 4.10, we find that the curves in $\mathcal{M}_{3}^{*}$ form an $\mathbb{R}$-invariant foliation $\mathcal{F}_{3}$ of $\mathbb{R} \times\left(M \backslash P_{\infty}\right)$, where

$$
M:=S^{3} \backslash \operatorname{int}\left(N_{1}^{\delta} \cup \cdots \cup N_{m}^{\delta}\right) .
$$

It projects to a smooth foliation of $M \backslash P_{\infty}$ by an $S^{1}$-parameterized family of leaves asymptotic to $P_{\infty}$ and transverse to $\partial M=L_{1} \cup \cdots \cup L_{m}$.

Definition 4.15 A foliation with the properties named above is called a stable holomorphic open book decomposition with boundary.

4.3.4 $\mathcal{F}_{3} \rightarrow \mathcal{F}_{4}$ : Twisting It follows from Remark 4.13 that there is a smooth homotopy of stable Hamiltonian structures $\mathcal{H}_{r}$ for $r \in[3,7 / 2]$, deforming $\mathcal{H}_{3}$ back to the original contact data $\mathcal{H}_{7 / 2}:=\left(\xi_{2}, X_{2}, d \lambda_{2}, J_{2}\right)$. Finally, continue this homotopy for $r \in[7 / 2,4]$ by choosing a smooth family of contact forms $\lambda_{r}$ on $M$ such that:

(1) $\lambda_{r} \equiv \lambda_{2}$ outside coordinate neighborhoods of the tori $L_{j}$, 
(2) in a coordinate neighborhood near $L_{j}, \lambda_{r}=f_{r}(\rho) d \theta+g_{r}(\rho) d \phi$ where $g_{r}^{\prime}(\rho)>0$ for all $r<4$ and $\rho \geq \delta$, but $g_{4}^{\prime}(\delta)=0$,

(3) $f_{4}$ and $g_{4}$ extend for $\rho$ in some open neighborhood of $\delta$ such that $g_{4}^{\prime \prime}(\delta)>0$ and $f_{4}^{\prime}(\delta)>0$.

These conditions guarantee that all closed Reeb orbits for $r<4$ are nontrivially linked with $P_{\infty}$, and this remains true at $r=4$ in int $M$, but the boundary components $L_{j}$ then become Morse-Bott tori with closed orbits forming negatively oriented meridians. Notice that no such homotopy of contact forms exists globally on $S^{3}$; this is why we introduced the boundary condition, to remove the interiors of $N_{j}^{\delta}$ from the picture.

For any $r_{0} \in(3,4)$, we can apply the arguments of Proposition 4.10 and find a continuous family $\mathcal{F}_{r}$ of stable holomorphic open book decompositions with boundary for $r \in$ $\left[3, r_{0}\right]$. Then taking $r \rightarrow 4$, the degeneration theorem (Theorem 3.5) gives limits in the form of finite energy surfaces without boundary, having $m+1$ positive punctures asymptotic to $P_{\infty}$ and the simply covered Morse-Bott orbits on $N_{1}^{\delta}, \ldots, N_{m}^{\delta}$. In particular, for any $m \in M \backslash\left(P_{\infty} \cup \partial M\right)$, there exists such a curve $\tilde{v}_{\infty}=\left(b_{\infty}, v_{\infty}\right)$ with $m$ in the image of $v_{\infty}$ : it is obtained by taking sequences of corresponding curves in $\mathcal{F}_{r}$ passing through $m$ and letting $r$ approach 4 . By positivity of intersections, the limit curves are also embedded, and any pair of them has projections that are either identical or disjoint in $M$. Finally, a simple computation using (6) and Lemma 2.5 shows that these curves have index 2 . They therefore constitute a stable finite energy foliation of Morse-Bott type on the manifold with boundary $M$. This, together with the constructions in Section 4.2, proves the main result for any situation in which Assumption 4.11 is satisfied.

\subsection{Lifting to general closed braids}

We now complete the proof of Theorem 1.1 by constructing foliations in cases where Assumption 4.11 does not hold. The idea is to define a branched cover over $S^{3}$ so that the assumption does hold on the cover, thus the previous arguments produce a foliation, which we will then show has a well defined projection.

By way of preparation, define the usual cylindrical coordinates $(\theta, \rho, \phi)$ on $M:=$ $S^{1} \times B_{\epsilon}^{2}(0)$, pick $n \in \mathbb{N}$ and consider the map

$$
p: S^{1} \times B_{\epsilon}^{2}(0) \rightarrow S^{1} \times B_{\epsilon}^{2}(0):(\theta, \rho, \phi) \mapsto(\theta, \rho, n \phi) .
$$

Writing $P:=S^{1} \times\{0\}$, this map is smooth on $M \backslash P$ and continuous everywhere. Suppose $M$ is endowed with a smooth contact form of type $\lambda=f(\rho) d \theta+g(\rho) d \phi$ 
and admissible complex multiplication defined by $J v_{1}=\beta(\rho) v_{2}$ as in Section 4.2. Then on $M \backslash P, \lambda$ pulls back to another contact form

$$
\lambda^{(n)}:=p^{*} \lambda=f_{n}(\rho) d \theta+g_{n}(\rho) d \phi=f(\rho) d \theta+n g(\rho) d \phi,
$$

which extends smoothly to $P$. In fact, let $\Phi_{0}$ be the trivialization of $\xi=\operatorname{ker} \lambda$ along $P$ defined by these coordinates, and suppose $P$ is a nondegenerate Reeb orbit for $\lambda$ with $\mu_{\mathrm{CZ}}^{\Phi_{0}}(P)=1$. From Proposition 4.6, this means $-f^{\prime \prime}(0) / 2 \pi g^{\prime \prime}(0) \in(0,1)$, and thus the same is true of $-f_{n}^{\prime \prime}(0) / 2 \pi g_{n}^{\prime \prime}(0)$, so $P$ also has $\mu_{\mathrm{CZ}}^{\Phi_{0}}(P)=1$ with respect to the extended contact form $\lambda^{(n)}$. Writing our standard symplectic frame on $\xi^{(n)}=\operatorname{ker} \lambda^{(n)}$ as $\left(v_{1}^{(n)}, v_{2}^{(n)}\right)$, the complex multiplication $J$ also pulls back on $M \backslash P$ to $J^{(n)}=p^{*} J$, satisfying $J^{(n)} v_{1}^{(n)}=\beta_{n}(\rho) v_{2}^{(n)}$ where $\beta_{n}(\rho)=\beta(\rho)$. It turns out that $J^{(n)}$ does not have a smooth extension over $P$, but this will be only a minor irritation in the following.

If Assumption 4.11 does not hold, choose $n$ to be the least common multiple of all the linking numbers $1 \mathrm{k}\left(K_{j}, P_{\infty}\right)$, and define an $n$-fold branched cover of $S^{3}$ as follows. By the results of Section 4.3.2, there is a contact form $\lambda_{2}$ and complex multiplication $J_{2}$, both of which take the usual simple forms in the neighborhoods $N_{j}$ of $K_{j}$, and $\left(S^{3}, \lambda_{2}, J_{2}\right)$ admits a stable finite energy foliation $\mathcal{F}_{2}$ which projects to an open book decomposition of $S^{3}$ with binding orbit $P_{\infty}$. Denoting $E:=S^{3} \backslash P_{\infty}$, this open book defines a fibration $E \rightarrow S^{1}$, and there is a natural $n$-fold covering map $p: E^{(n)} \rightarrow E$ and smooth fibration $E^{(n)} \rightarrow S^{1}$ such that $p\left(E_{\tau}^{(n)}\right)=E_{n \tau}$ for each $\tau \in S^{1}$. Let $\psi: E^{(n)} \rightarrow E^{(n)}$ be the deck transformation which maps

$$
E_{\tau}^{(n)} \rightarrow E_{\tau+\frac{1}{n}}^{(n)}
$$

then every deck transformation is of the form $\psi^{k}$ for $k \in \mathbb{Z}_{n}$.

In order to compactify $E^{(n)}$, we shall modify this construction carefully near $P_{\infty}$. Recall from (45) that a neighborhood of $P_{\infty}$ admits cylindrical polar coordinates $(\theta, \rho, \phi)$ such that $P_{\infty} \cong S^{1} \times\{0\}$ and $\lambda_{2}$ takes the form

$$
\lambda_{2}=f(\rho) d \theta+g(\rho) d \phi,
$$

where $f$ and $g$ are smooth functions with $f^{\prime} g^{\prime \prime}-f^{\prime \prime} g^{\prime} \equiv 0$ near 0 . Moreover $J_{2}$ is defined in this neighborhood by a relation of the form $J_{2} v_{1}=\beta(\rho) v_{2}$. These properties continue to hold if we change the coordinates by any diffeomorphism of the form $(\theta, \rho, \phi) \longleftrightarrow(\theta, \rho, \phi+2 \pi k \theta)$ for $k \in \mathbb{Z}$, thus we can assume without loss of generality that the planes in $\mathcal{F}_{2}$ have trivial winding around $P_{\infty}$ as they approach it in these coordinates. In this case the coordinates define a trivialization $\Phi_{0}$ in which $\mu_{\mathrm{CZ}}^{\Phi_{0}}\left(P_{\infty}\right)=1$. We can now replace $\mathcal{F}_{2}$ with another (homotopic) open book 
decomposition whose pages look like $\{\phi=$ const $\}$ in a neighborhood of $P_{\infty}$. Then applying the covering construction above, $E^{(n)}$ admits a compactification

$$
\bar{E}^{(n)}=E^{(n)} \cup P_{\infty}^{(n)} \cong S^{3},
$$

where $P_{\infty}^{(n)}$ is a circle identified with $S^{1} \times\{0\}$ in a certain cylindrical coordinate neighborhood, and $p$ maps this neighborhood to a neighborhood of $P_{\infty}$ via (47).

There is thus a continuous extension $p: \bar{E}^{(n)} \rightarrow S^{3}$, such that $\lambda^{(n)}=p^{*} \lambda_{2}$ has the form (48) and thus extends smoothly over $\bar{E}^{(n)}$; in fact for this extension, $P_{\infty}^{(n)}$ is a nondegenerate Reeb orbit with $\mu_{\mathrm{CZ}}^{\Phi_{0}}\left(P_{\infty}^{(n)}\right)=1$. The lift $J^{(n)}=p^{*} J_{2}$ is uniquely defined over $E^{(n)}$ but singular at $P_{\infty}^{(n)}$. These are both preserved by the deck transformation $\psi$, and together they define an almost complex structure $\widetilde{J}^{(n)}$ on $\mathbb{R} \times E^{(n)} \subset \mathbb{R} \times \bar{E}^{(n)}$, such that the diffeomorphism

$$
\tilde{\psi}: \mathbb{R} \times E^{(n)} \rightarrow \mathbb{R} \times E^{(n)}:(a, m) \mapsto(a, \psi(m))
$$

is $\widetilde{J}^{(n)}$-holomorphic. Each leaf $\tilde{u} \in \mathcal{F}_{2}$ lifts to an embedded $\widetilde{J}^{(n)}$-holomorphic plane $\mathbb{C} \rightarrow \mathbb{R} \times E^{(n)}$, giving a foliation of $E^{(n)}$ by planes asymptotic to $P_{\infty}^{(n)}$. These can be made into an honest stable foliation by changing $\beta_{n}(\rho)$ for $\rho$ near 0 so that $J^{(n)}$ becomes smooth. This is possible by Proposition 4.7, because $f_{n}^{\prime} g_{n}^{\prime \prime}-f_{n}^{\prime \prime} g_{n}^{\prime} \equiv 0$ in this neighborhood: thus for a suitable smooth choice of $J^{(n)}$, we obtain a stable foliation $\mathcal{F}_{2}^{(n)}$ which matches the original lift of $\mathcal{F}_{2}$ outside some neighborhood of $P_{\infty}^{(n)}$.

Here is the main point: the link $K \subset E$ lifts to another transverse link $K^{(n)}=p^{-1}(K) \subset$ $E^{(n)}$, and our choice of $n$ guarantees that every connected component $K_{j}^{(n)} \subset K^{(n)}$ satisfy $\operatorname{lk}\left(K_{j}^{(n)}, P_{\infty}^{(n)}\right)=1$ (see Figure 15). Thus the arguments of Section 4.3.3 and Section 4.3.4 produce a stable finite energy foliation $\mathcal{F}_{4}$ of Morse-Bott type on $\left(M^{(n)}, \lambda_{4}^{(n)}, J_{4}^{(n)}\right)$, where $M^{(n)}$ is the complement of a neighborhood $N^{(n)}$ of $K^{(n)}$ in $\bar{E}^{(n)} \cong S^{3}$. We can easily arrange moreover that $N^{(n)}, \lambda_{4}^{(n)}$ and $J_{4}^{(n)}$ be invariant under the deck transformation $\psi$, so the diffeomorphisms $\tilde{\psi}^{k}$ for $k \in \mathbb{Z}_{n}$ are $\widetilde{J}_{4}^{(n)}$-holomorphic. This gives rise to a set of $n$ stable foliations $\mathcal{F}_{4}^{k}:=\tilde{\psi}^{k}\left(\mathcal{F}_{4}\right)$ for $k \in \mathbb{Z}_{n}$.

Proposition 4.16 The foliations $\mathcal{F}_{4}^{k}$ are all identical.

Proof It suffices to show that for any leaf $\tilde{u}=(a, u) \in \mathcal{F}_{4}$, the curve

$$
\tilde{\psi} \circ \tilde{u}=(a, \psi \circ u): \dot{\Sigma} \rightarrow \mathbb{R} \times M^{(n)}
$$

is also a leaf of the foliation. This follows from positivity of intersections. Indeed, if $\tilde{\psi} \circ \tilde{u}$ is not a leaf, it must have finitely many isolated intersections with some other 

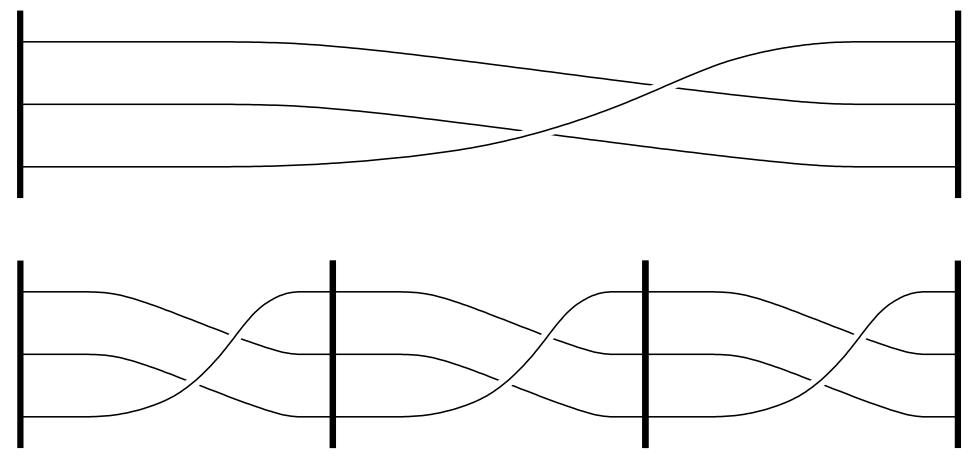

Figure 15: The top is a transverse knot $K$ with $\operatorname{lk}\left(K, P_{\infty}\right)=3$, represented as a closed braid. The bottom is the 3 -fold cover $K^{(3)} \subset E^{(3)}$, with three components cyclically permuted by $\psi$.

leaf $\tilde{v}=(b, v) \in \mathcal{F}_{4}$, and these cannot be eliminated under homotopies. Thus $\tilde{\psi} \circ \tilde{u}$ also has isolated intersections with $\tilde{u}$. Now applying an $\mathbb{R}$-translation, $\tilde{\psi} \circ \tilde{u}$ also intersects $\widetilde{u}^{\sigma}:=(a+\sigma, u)$ for all $\sigma \in \mathbb{R}$. Since $a: \dot{\Sigma} \rightarrow \mathbb{R}$ is a proper map, choosing $\sigma$ large forces these intersections toward the asymptotic limits. But $\tilde{u}$ and $\tilde{\psi} \circ \tilde{u}$ clearly have distinct asymptotic limits, and neither curve intersects the asymptotic limits of the other, thus we have a contradiction.

It follows that $\mathcal{F}_{4}$ has a well defined projection under $p$ to an $\mathbb{R}$-invariant foliation of $\mathbb{R} \times S^{3}$, inducing also a foliation of $S^{3} \backslash P_{\infty}$ by planes asymptotic to $P_{\infty}$. The projection of $J_{4}$ is singular at $P_{\infty}$, but this can again be fixed by an application of Proposition 4.7. The proof of Theorem 1.1 is now complete.

\section{References}

[1] C Abbas, Holomorphic open book decompositions In preparation

[2] D Bennequin, Entrelacements et équations de Pfaff, from: “Third Schnepfenried geometry conference, Vol. 1 (Schnepfenried, 1982)", Astérisque 107, Soc. Math. France, Paris (1983) 87-161 MR753131 Translated in Russian Math. Surveys, 44(3) (1989) $1-65$

[3] F Bourgeois, Y Eliashberg, H Hofer, K Wysocki, E Zehnder, Compactness results in symplectic field theory, Geom. Topol. 7 (2003) 799-888 MR2026549

[4] Y Eliashberg, Classification of overtwisted contact structures on 3-manifolds, Invent. Math. 98 (1989) 623-637 MR1022310 
[5] Y Eliashberg, A Givental, H Hofer, Introduction to symplectic field theory, Geom. Funct. Anal. (2000) 560-673 MR1826267

[6] J B Etnyre, Planar open book decompositions and contact structures, Int. Math. Res. Not. (2004) 4255-4267 MR2126827

[7] H Geiges, Contact geometry, from: "Handbook of differential geometry. Vol. II", Elsevier/North-Holland, Amsterdam (2006) 315-382 MR2194671

[8] E Giroux, Lecture given at Georgia Topology conference (2001) Notes available at http://www.math.uga.edu/ topology/2001/giroux.pdf

[9] E Giroux, Géométrie de contact: de la dimension trois vers les dimensions supérieures, from: "Proceedings of the International Congress of Mathematicians, Vol. II (Beijing, 2002)", Higher Ed. Press, Beijing (2002) 405-414 MR1957051

[10] H Hofer, Pseudoholomorphic curves in symplectizations with applications to the Weinstein conjecture in dimension three, Invent. Math. 114 (1993) 515-563 MR1244912

[11] H Hofer, K Wysocki, E Zehnder, Properties of pseudoholomorphic curves in symplectisations. I. Asymptotics, Ann. Inst. H. Poincaré Anal. Non Linéaire 13 (1996) 337-379 MR1395676

[12] H Hofer, K Wysocki, E Zehnder, Properties of pseudo-holomorphic curves in symplectisations. II. Embedding controls and algebraic invariants, Geom. Funct. Anal. 5 (1995) 270-328 MR1334869

[13] H Hofer, K Wysocki, E Zehnder, Properties of pseudoholomorphic curves in symplectizations. III. Fredholm theory, from: "Topics in nonlinear analysis", Progr. Nonlinear Differential Equations Appl. 35, Birkhäuser, Basel (1999) 381-475 MR1725579

[14] H Hofer, K Wysocki, E Zehnder, Properties of pseudoholomorphic curves in symplectisation. IV. Asymptotics with degeneracies, from: "Contact and symplectic geometry (Cambridge, 1994)", Publ. Newton Inst. 8, Cambridge Univ. Press, Cambridge (1996) 78-117 MR1432460

[15] H Hofer, K Wysocki, E Zehnder, A characterisation of the tight three-sphere, Duke Math. J. 81 (1995) 159-226 (1996) MR1381975

[16] H Hofer, K Wysocki, E Zehnder, Finite energy foliations of tight three-spheres and Hamiltonian dynamics, Ann. of Math. (2) 157 (2003) 125-255 MR1954266

[17] H Hofer, E Zehnder, Symplectic invariants and Hamiltonian dynamics, Birkhäuser Advanced Texts: Basler Lehrbücher. [Birkhäuser Advanced Texts: Basel Textbooks], Birkhäuser Verlag, Basel (1994) MR1306732

[18] C Hummel, Gromov's compactness theorem for pseudo-holomorphic curves, Progress in Mathematics 151, Birkhäuser Verlag, Basel (1997) MR1451624

[19] W B R Lickorish, A representation of orientable combinatorial 3-manifolds, Ann. of Math. (2) 76 (1962) 531-540 MR0151948 
[20] R Lutz, Sur quelques propriétés des formes différentielles en dimension trois, $\mathrm{PhD}$ thesis, Strasbourg (1971)

[21] R Lutz, Structures de contact sur les fibrés principaux en cercles de dimension trois, Ann. Inst. Fourier (Grenoble) 27 (1977) ix, 1-15 MR0478180

[22] J Martinet, Formes de contact sur les variétés de dimension 3, from: "Proceedings of Liverpool Singularities Symposium, II (1969/1970)”, Springer, Berlin (1971) 142-163. Lecture Notes in Math., Vol. 209 MR0350771

[23] D McDuff, D Salamon, J-holomorphic curves and symplectic topology, American Mathematical Society Colloquium Publications 52, American Mathematical Society, Providence, RI (2004) MR2045629

[24] N Saveliev, Lectures on the topology of 3-manifolds. An introduction to the Casson invariant, de Gruyter Textbook, Walter de Gruyter \& Co., Berlin (1999) MR1712769

[25] M Seppälä, T Sorvali, Geometry of Riemann surfaces and Teichmüller spaces, volume 169 of North-Holland Mathematics Studies, North-Holland Publishing Co. (1992)

[26] R Siefring, Intersection theory of finite energy surfaces, PhD thesis, New York University (2005)

[27] A H Wallace, Modifications and cobounding manifolds, Canad. J. Math. 12 (1960) 503-528 MR0125588

[28] C Wendl, Punctured holomorphic curves with boundary in 3-manifolds: Fredholm theory and embededdness In preparation

[29] C Wendl, Finite energy foliations and surgery on transverse links, $\mathrm{PhD}$ thesis, New York University (2005)

[30] R Ye, Filling by holomorphic curves in symplectic 4-manifolds, Trans. Amer. Math. Soc. 350 (1998) 213-250 MR1422913

Departement Mathematik, HG G38.1, Rämistrasse 101 8092 Zürich, Switzerland

wendl@math.ethz.ch

http://www . math.ethz.ch/ wendl/

Proposed: Yasha Eliashberg

Seconded: Leonid Polterovich and Simon Donaldson
Received: 19 November 2006

Accepted: 20 December 2007 